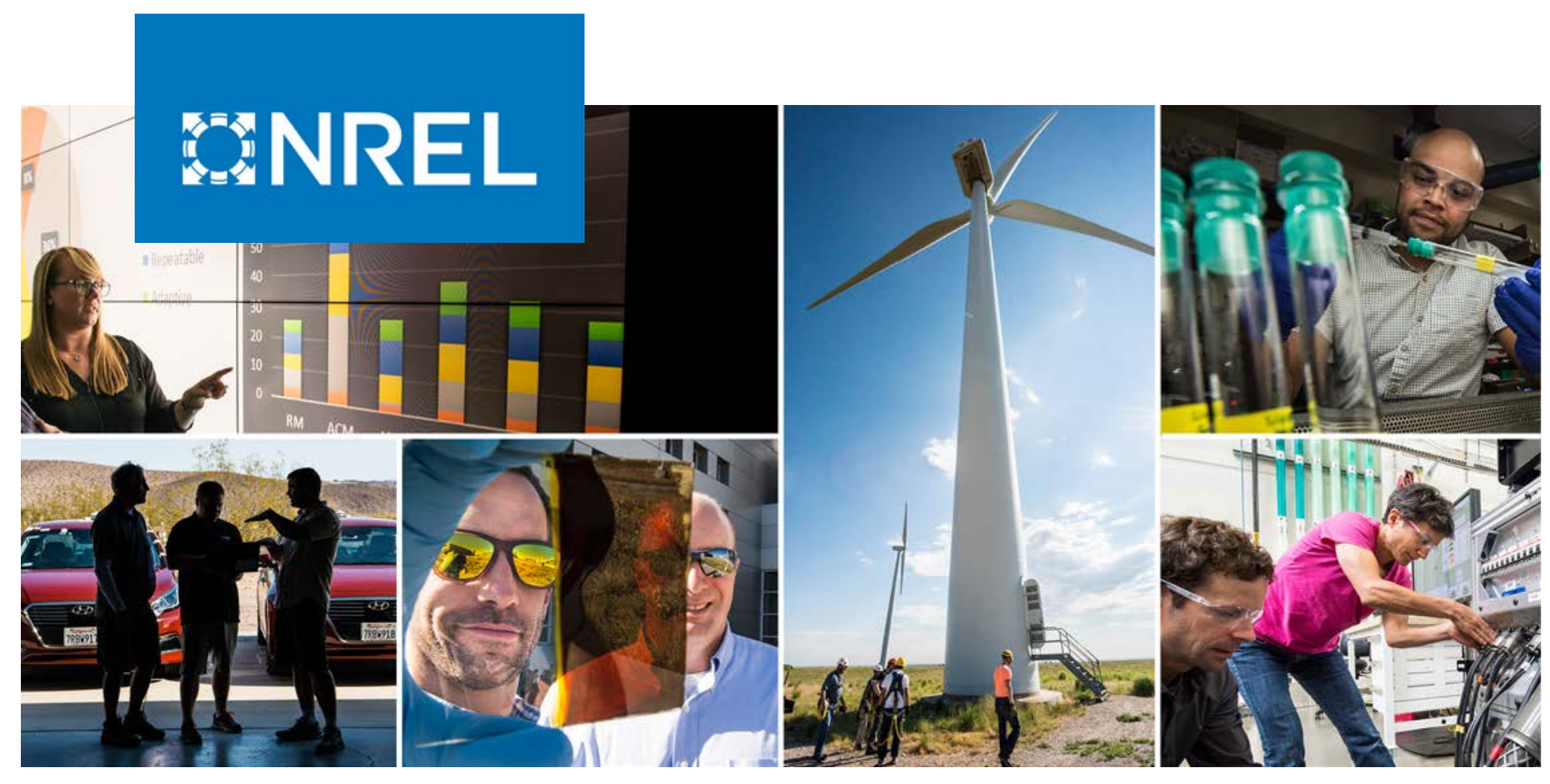

\title{
Load Forecasting for the Moroccan Electricity Sector
}

Jordan Cox, Thushara de Silva, Jennie Jorgenson, and Barbara O'Neill

National Renewable Energy Laboratory

NREL is a national laboratory of the U.S. Department of Energy

Office of Energy Efficiency \& Renewable Energy

Operated by the Alliance for Sustainable Energy, LLC

This report is available at no cost from the National Renewable Energy Laboratory (NREL) at www.nrel.gov/publications.
Technical Report

NREL/TP-5R00-77427

August 2021 


\section{GHREL}

\section{Load Forecasting for the Moroccan Electricity Sector}

Jordan Cox, Thushara de Silva, Jennie Jorgenson, and Barbara O'Neill

National Renewable Energy Laboratory

\section{Suggested Citation}

Cox, Jordan, Thushara de Silva, Jennie Jorgenson, and Barbara O'Neill. 2021. Load Forecasting for the Moroccan Electricity Sector. Golden, CO: National Renewable Energy Laboratory. NREL/TP-5R00-77427. https://www.nrel.gov/docs/fy21osti/77427.pdf.

NREL is a national laboratory of the U.S. Department of Energy Office of Energy Efficiency \& Renewable Energy Operated by the Alliance for Sustainable Energy, LLC

This report is available at no cost from the National Renewable Energy Laboratory (NREL) at www.nrel.gov/publications.

Contract No. DE-AC36-08GO28308
Technical Report

NREL/TP-5R00-77427

August 2021

National Renewable Energy Laboratory 15013 Denver West Parkway Golden, CO 80401 303-275-3000 • www.nrel.gov 


\section{NOTICE}

This work was authored by the National Renewable Energy Laboratory, operated by Alliance for Sustainable Energy, LLC, for the U.S. Department of Energy (DOE) under Contract No. DE-AC36-08GO28308. Funding provided by U.S. Department of Energy Office of International Affairs and U.S. Department of State. The views expressed herein do not necessarily represent the views of the DOE or the U.S. Government.

This report is available at no cost from the National Renewable Energy Laboratory (NREL) at www.nrel.gov/publications.

U.S. Department of Energy (DOE) reports produced after 1991 and a growing number of pre-1991 documents are available free via www.OSTI.gov.

Cover Photos by Dennis Schroeder: (clockwise, left to right) NREL 51934, NREL 45897, NREL 42160, NREL 45891, NREL 48097, NREL 46526.

NREL prints on paper that contains recycled content. 


\section{Acknowledgments}

The work included in this report would not have been possible without partners of the Moroccan Office National de l'Electricité et de l'Eau Potable (ONEE). ONEE has graciously hosted and assisted the National Renewable Energy Laboratory (NREL) team with data, advice, and feedback on the work herein.

The authors thank Mr. Abderrahim EL Hafidi, Director General of ONEE for leading and supporting this cooperation project for ONEE.

In addition, to the authors are grateful to (in no order) Mohammed Bouramtane, Brahim Oumounah, Abdelghani Hammadia, Khalid Mohammed Nejjar, Soufiane Hachad, Loumia Mellouki Felali, Amal Bouamama, and Youssef Kettani, also of ONEE.

The NREL team would also like to thank Ryan Guirlinger and Mustapha ElGamra of the U.S. Embassy in Morocco for providing aid multiple times during this project.

Finally, the NREL team especially wishes to thank Ray Behbehani of the U.S. Department of Energy, who enabled and guided this work. This report would not have been possible without him. 


\section{Executive Summary}

Load forecasts are an important tool for decision makers to ensure reliable energy systems. Load forecasting seeks to understand and predict future energy demand at relevant timescales and provide decision makers with information and assumptions to decide whether to procure new resources when necessary. Load forecasting generally takes place on the long ( $>24$ months), medium (1-24 months) and short (days) timescales or terms (Hahn, Meyer-Nieberg, and Pick1 2009). Load forecasting timescales are matched to decision-making around building new power plants (long), servicing power plants and starting new energy saving programs (often medium) and calling on individual generators (or consumers) to meet (or reduce) demand (short). Load forecasting becomes particularly important as variable energy is increasingly utilized. Variable energy sources like wind and solar photovoltaics (PV) can be operated reliably, but they are most economic when forecasts of both weather and electricity demand are sufficiently accurate and timely to ensure that supply meets demand on the hourly, seasonal, and annual timescales.

In 2015 Morocco announced its intention to have 52\% of electricity generation capacity from renewable sources (20\% solar, 20\% wind, and 12\% hydroelectric) by 2030 ("Morocco Renewable Energy Target 2030 - Policies" 2019). The country has made great strides in this area. For example, the largest concentrating solar power plant in the world is the Ouarzazate plant (units I, II, and III), which is located in southeast Morocco and was completed in 2018. As of the end of 2019, Morocco had installed 1.22 GW of wind, 711 MW of solar, and 1,770 MW of hydroelectricity, including $464 \mathrm{MW}$ in pumped-storage hydropower, with specific regulatory changes enacted to encourage uptake (such as allowing for competition from independent power producers in the renewable energy sector). Baseload energy is still primarily served by coal ("Rapport D’Activités 2019 Energie Electrique" 2019; "Chiffre Clés 2019 Energie Electrique" 2019).

On the demand side, Morocco has seen steadily increasing needs for power generation. On average, power demand grew $6.5 \%$ annually between 2003 and 2014, primarily due to economic growth, increased industrial load, and rural electrification. There was a certain deceleration between 2015 and 2019 when demand only grew by 3.1\% on average due to a slight decline in economic growth, energy efficiency, and electrification load growth being saturated, reaching a rate of $99.72 \%$ at the end of 2019 ("ONEE Data, Site Web Officiel de l'ONEE - Branche Electricité" 2021). Morocco categorizes its utility customers into residential, commercial, public lighting, administration, industrial, and agricultural. Already substantial, the residential sector has increased its share over time. This likely relates to urbanization and increased use of air conditioning.

This report contributes to the building of robust load forecasting capabilities within Morocco. The Office National de 1'Electricité et de l'Eau Potable (ONEE, or Moroccan Office of Electricity and Drinking Water) and the National Renewable Energy Laboratory (NREL), supported by the U.S. Department of Energy and U.S. Department of State, worked together to conduct load forecasting on the long, medium, and short terms for the electricity system. ONEE had already conducted some long-term load forecasts based on scenarios of population and economic growth. This report builds on ONEE's data and expertise to test several algorithms related to regression or multivariable regression, decomposition, auto-regressive, and machine learning to predict future electricity demand. 
For the three timescales described a total of eight methodologies were tested (three for longterm, three for medium-term, and two for short-term), six of which were included in this report. These methodologies were linear and multivariable regression on the annual (long-term) and hourly (long- and medium-term) basis, load clustering (medium-term), and two auto regressive models for short-term analysis.

Based on long-term analysis, depending on growth scenarios, Morocco can expect an annual peak demand in 2030 ranging approximately 8-11 GW with annual energy consumption ranging 55-77 TWh. This is based on an assumed annual gross domestic product (GDP) growth of $2.5 \%-4.0 \%$. Analysis was conducted for PV deployment between 1-7 GW and resulting net load curves are forecast. See Section 9.2. Additional work will need to be done to evaluate system integration potential, depending on the geospatial placement of PV and other renewable energy (RE) and non-RE deployments.

For the medium term, several regressions were tested and produced hourly load forecasts for one year in advance. Using historical data, three methodologies were tested and were found to have an average error of $6 \%-12 \%$. Additionally, the Moroccan power system was compared to other jurisdictions in terms of load factor and system efficiency through the evaluation of load duration curves, as discussed in Section 9.3.2. Included in this report is a comparison to the Electric Reliability Council of Texas (ERCOT) system which was shown to have a flatter load duration curve, but a higher ratio of minimum to maximum system load. In contrast, since 2011, the Moroccan power system has decreased its system maximum to minimum load ratio to 2.25, indicating a more efficient system, though there are still opportunities to increase system load factor and flatten the load curve. The Electric Reliability Council of Texas was chosen as an appropriate comparison due to its similar temperature and geographic parameters, as is further discussed in Section 9.2.

For the short-term analysis, artificial intelligence-driven AR methods were used in conjunction with historical data to predict electricity demand over a one-week period using three regimes: a Monday, Tuesday, Wednesday, Thursday model, a Friday-Saturday model, and a Sunday model. These methods showed a mean error of approximately $1 \%-3 \%$ and a maximum error of $6 \%-$ $13 \%$. This short-term methodology could potentially be deployed in real time by a central agency to produce demand forecasts.

The load forecasting methodologies listed in this report all showed promise and encouraging results; however, as conducted, the errors and uncertainties for these methods were larger than would be desirable for operational load forecasts to guide Moroccan decision makers. Errors should be reduced such that reserve capacity can compensate for errors to prevent the need for load shedding and to maximize system reliability, while also minimizing system cost. The following recommendations were identified as possible methods to reduce load forecasting error:

- Establish a data acquisition plan: Based on the uncertainties observed in this work, it is recommended that ONEE develop a plan to collect more granular electricity load data to reduce the most impactful uncertainties. Data that could be of most use includes the customer, city, and region level electricity demand with higher temporal resolution, and a longer chronological duration to account for meteorological years. 
- Develop Morocco-specific load profiles and scenarios: The load forecasting performed in this report was top-down, meaning it focused on "macro" trends of the aggregate electricity load. An alternative method is bottom-up load forecasting that uses standardized curves for electricity customers (such as residential, commercial, etc.) and aggregates them to predict not just total but also sector-based demand. This bottom-up methodology can then examine scenarios, such as widespread air conditioning adoption, and how these scenarios could impact electricity demand. A recommended next step would be to identify key sectors of the Moroccan economy, build standardized load profiles, and reexamine load forecasts in this report using a scenario-based approach.

- Test alternative models and algorithms: In the literature cited for this work, there are additional mathematical models for producing load forecasts. Many of these advanced methods rely on further segmenting the data into categories such as winter-weekday, winter-weekend, winter-holiday, summer-weekday, summer-weekend, summer-holiday, etc. Beginning with the peak season on the Moroccan grid, it is recommended that future load forecasts further split the data into representative categories and examine additional models and algorithms.

- Establish a best practice with regards to weather data: The load forecasting in this report examined weather data as it related to the short-term electricity demand; however, the weather data used in this report was oversimplified as part of the initial attempt. Building a more robust load forecast would require a better understanding of the interplay between weather and electricity demand. ONEE could therefore consider launching an indepth study about this relationship and develop a list of best practices (i.e., weighting local weather measurements by population) to process and connect weather data to electricity load that can be used across load forecasting efforts. Ultimately, weather data will be linked to both customer consumption (such as heating and air conditioning) and customer production (for rooftop solar PV generation). Understanding the relationship between weather and electricity at a higher geographic, relational, and temporal resolution is recommended for further study.

- Establish a load forecasting authority: Load forecasting on multiple timescales has large data requirements and is generally undertaken by a central authority such as utilities or government entities (Hong 2014; 2016; Kuster, Rezgui, and Mourshed 2017; Singh and Khatoon 2012). This central authority then communicates its load forecasts with other relevant entities. This report makes no recommendations on the organization, structure, or authority that should take on load forecasting responsibilities within Morocco.

As Morocco pursues its ambitious clean energy goals, ONEE has a central role to play in load forecasting and has a unique opportunity to develop novel methodologies for load forecasting in North Africa. There are many unique aspects of this region that have yet to be examined in terms of electricity load forecasting. Exploring the effects on electricity load of local variables could establish ONEE as a regional leader in this space and contribute to national research and development activity goals. 


\section{List of Acronyms}

$\mathrm{ACF}$

AIC

AMEE

ANRE

ARIMA

ARMA

BIC

GDP

$\mathrm{HCP}$

IRESEN

LEAP

MAED

MAGG

MASEN

MEFAR

MEME

MTWR

NGCP

NREL

ONEE

PACF

PDOE

PLF

PV

SARIMA

SARIMAX

SIE

UPME

UN

VRE autocorrelation function

Akaike information criterion

Moroccan Agency for Energy Efficiency

Moroccan Electricity Regulatory Authority

auto regressive integrated moving average

auto regressive moving average

Bayesian information criterion

gross domestic product

Moroccan High Commission for Planning

Institute for Research into Solar and Renewable Energies

Long-range Energy Alternatives Planning

Model for Analysis of Energy Demand

Ministry of General Affairs and Governance

Moroccan Agency for Sustainable Energy

Minister of the Economy, Finance and Administration Reform

Ministry of Energy, Mines and Environment

Monday, Tuesday, Wednesday, Thursday

National Grid Corporation of the Philippines

National Renewable Energy Laboratory

Office National de l'Electricité et de l'Eau Potable, Moroccan Electricity and Drinking Water utility

partial autocorrelation function

Philippines Department of Energy

probabilistic load forecasting

photovoltaic

seasonal auto regressive integrated moving average

seasonal auto regressive integrated moving average with exogenous

variable

Société d'Investissement Energétiques

National Mining and Energy Planning Unit

United Nations

variable renewable energy 


\section{Table of Variables}

Throughout the formulas and calculations in this report, several variables are used. The table below provides these variables along with their definitions and units.

\begin{tabular}{|c|l|l|}
\hline Parameter & Definition & Units \\
\hline$L$ & System load & megawatts (MW) \\
\hline$y$ & Year & - \\
\hline$t$ & Hour of year (1-8,760) & - \\
\hline$P$ & Peak system load & MW \\
\hline$A$ & Annual system load & $\begin{array}{l}\text { megawatt-hours (MWh), } \\
\text { gigawatt-hours (GWh), and } \\
\text { terawatt-hours (TWh) }\end{array}$ \\
\hline$a, b, c, d$ & Regression coefficients & - \\
\hline$L_{n o r m}$ & $\begin{array}{l}\text { System load normalized (value between 0 and 1) to } \\
\text { peak load for a time domain }\end{array}$ & - \\
\hline$h$ & Hour of the day (1-24) & - \\
\hline$S$ & Solar generation & MW \\
\hline$L_{n e t}$ & $\begin{array}{l}\text { Net load (system load less contributions from solar } \\
\text { and/or wind) }\end{array}$ & MW \\
\hline$R$ & System ramp rate & MW/time period \\
\hline $\begin{array}{c}p, d, q, \\
P, D, Q\end{array}$ & SARIMAX model parameters & $\begin{array}{l}\text { Units provided in model } \\
\text { description. }\end{array}$ \\
\hline$\epsilon, \theta, C$ & $\begin{array}{l}\text { Error terms, coefficients, and constants used in the } \\
\text { SARIMAX model }\end{array}$ & $\begin{array}{l}\text { Units provided in model } \\
\text { description. }\end{array}$ \\
\hline
\end{tabular}




\section{Table of Contents}

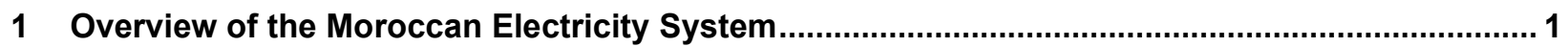

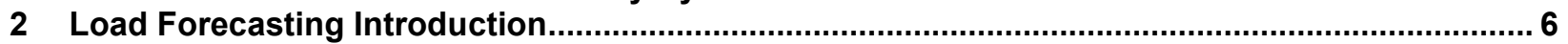

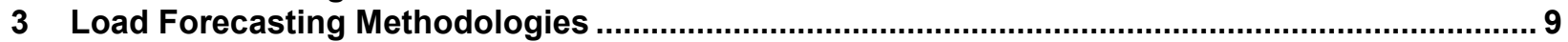

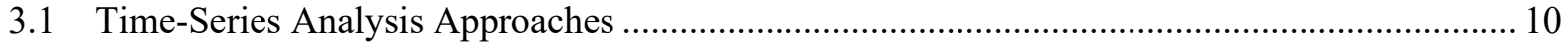

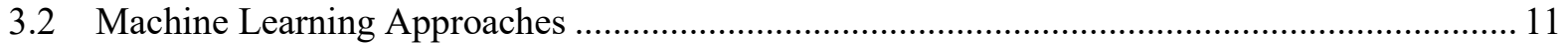

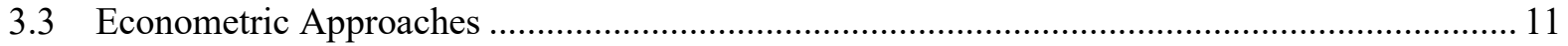

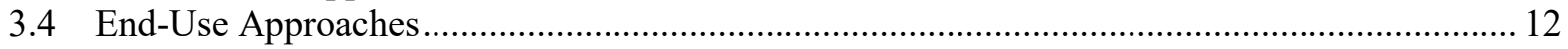

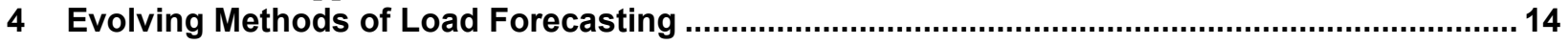

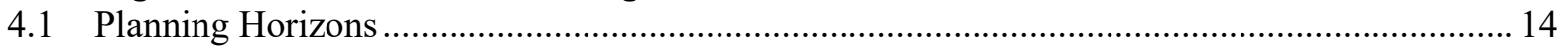

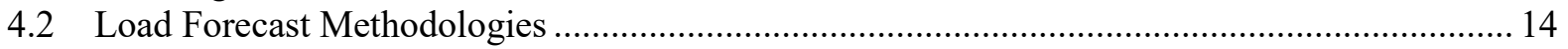

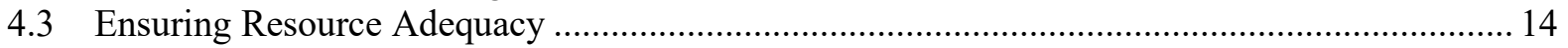

4.4 Temporal and Spatial Resolution of Load Forecasts............................................................... 15

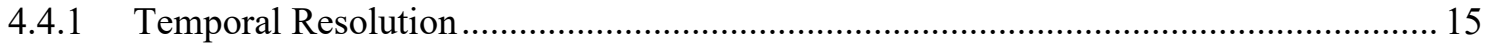

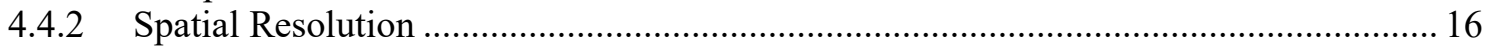

5 Case Studies of Long-Term Load Forecasting in Select Developing Countries ...................... 17

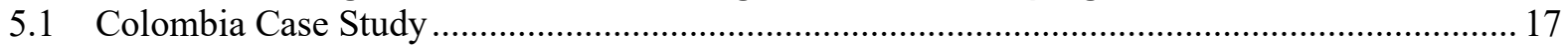

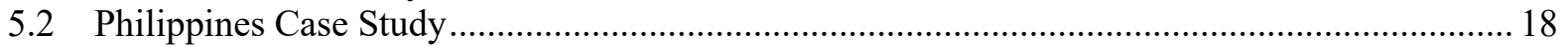

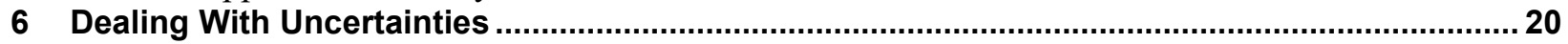

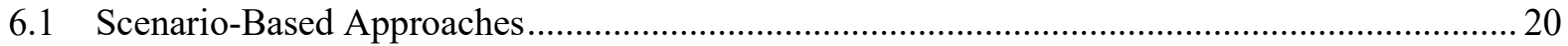

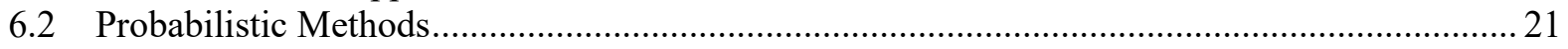

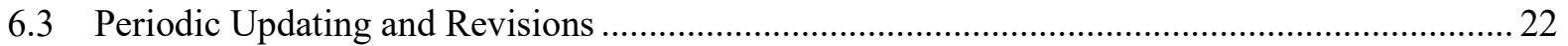

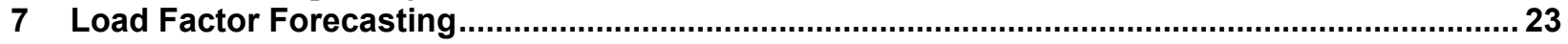

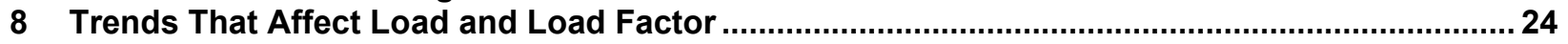

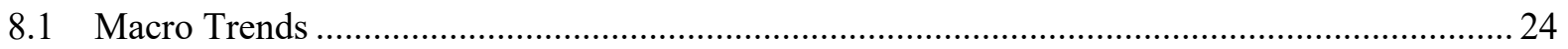

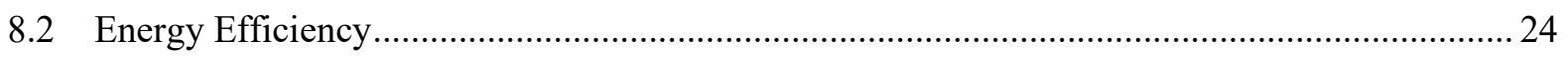

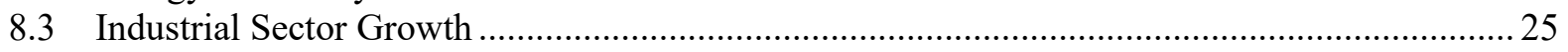

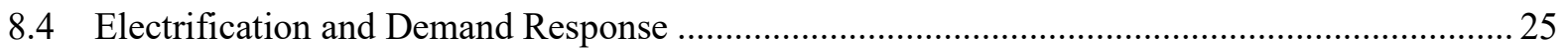

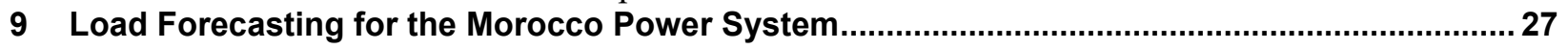

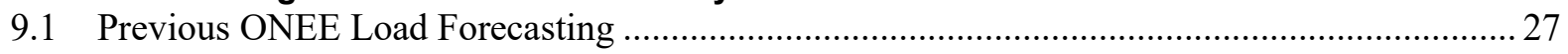

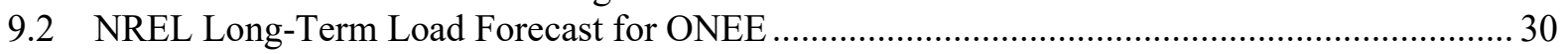

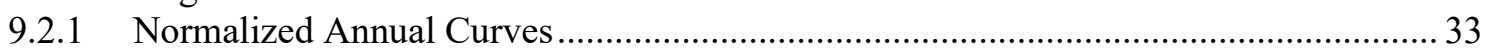

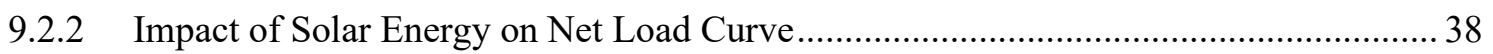

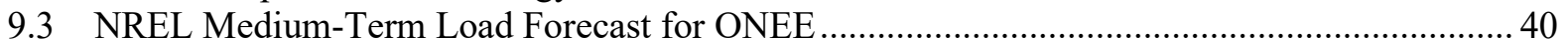

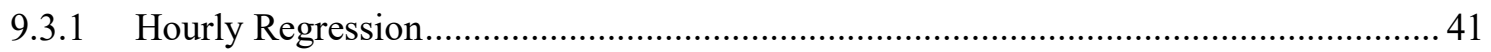

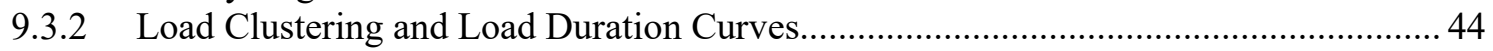

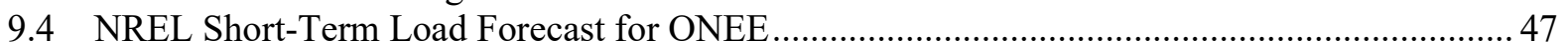

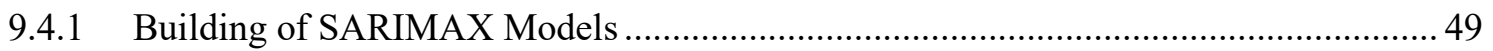

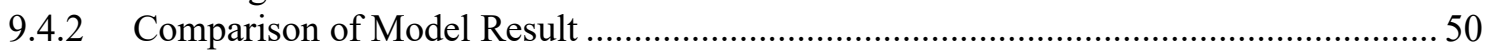

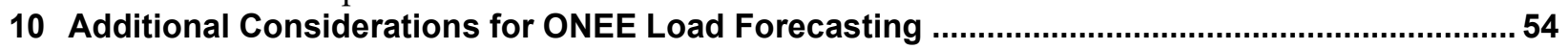

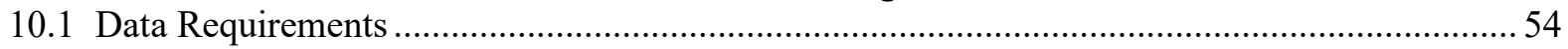

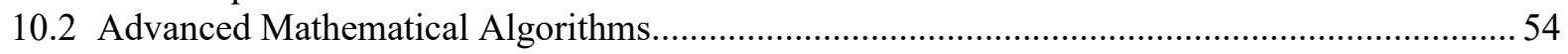

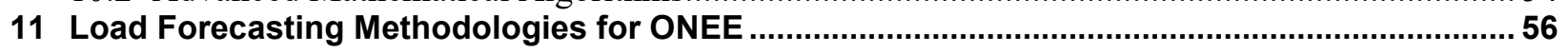

11.1 Impacts of Energy Efficiency in Long-Term Load Forecasting............................................ 56

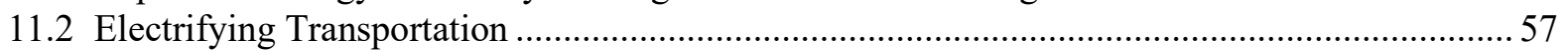

11.3 Impacts of Other Technology Deployment in Long-Term Load Forecasting ........................... 58

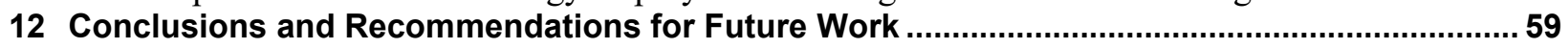

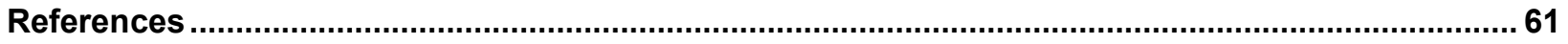




\section{List of Figures}

Figure 1. Electricity generation by source, Morocco 1990-2017 f........................................................ 1

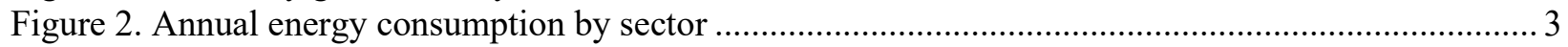

Figure 3. Private and public entities in the energy sector and their roles ............................................ 5

Figure 4. Key steps of traditional power system planning processes (orange boxes) and additional steps

for integrating variable renewable energy (green boxes) ....................................................... 7

Figure 5. Peak demand forecast approach for the Philippine Power Development Plan.......................... 19

Figure 6. Peak system demand growth as forecasted by ONEE ......................................................... 29

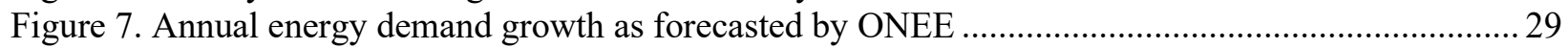

Figure 8. Three projected levels of GDP for Morocco through 2030 ..................................................... 30

Figure 9. Linear and logarithmic regression of GDP, peak load, and annual energy consumption............ 31

Figure 10. Peak load versus GDP regression for low, medium, and high growth .................................. 31

Figure 11. Annual energy consumption versus GDP regression for low, medium, and high growth......... 32

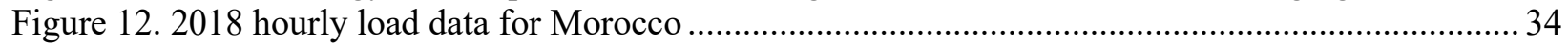

Figure 13. 2018-2030 Low (Green), Medium (Blue), and High (Red) hourly load curves derived from

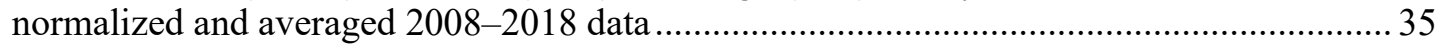

Figure 14. 2018 normalized monthly load curves for Morocco............................................................ 36

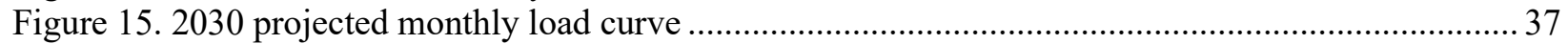

Figure 16. Monthly average forecast load curves and projected solar output based on installed capacity. 39

Figure 17. Monthly average forecast load curves and forecast net load based on installed solar capacity 40

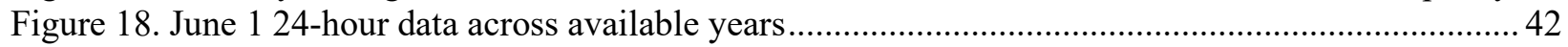

Figure 19. June 124 -hour data across available years with linear regression ........................................ 43

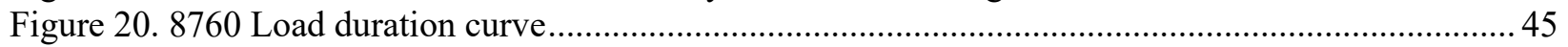

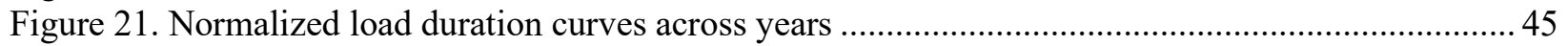

Figure 22. Normalized load duration curves across years with Texas comparison ................................... 46

Figure 23. Hourly electricity demand of the Morocco Year 2018, December......................................... 48

Figure 24. Hourly time series load data series for 2015-2018 demonstrates significant (a) autocorrelation and (b) partial correlation to the few lag hourly data electricity demand. ............................ 49

Figure 25. Forecasting of seven days ahead using three models of SARIMA for Monday to Thursday (MTWR), Friday and Saturday (FS), and Sunday, built with (a) 2015-2018 hourly temperature and load, (b) 2015-2018 hourly load data, (c) 2018 hourly temperature and load,

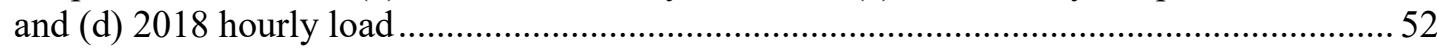

\section{List of Tables}

Table 1. Key Stakeholders in the Moroccan Electricity Sector ............................................................... 4

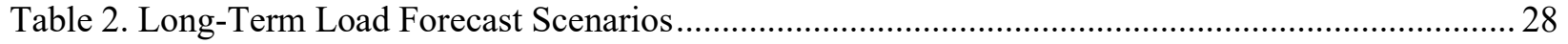

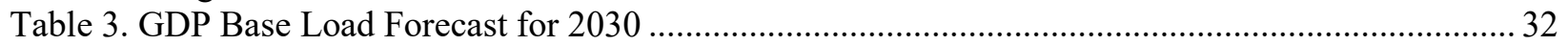

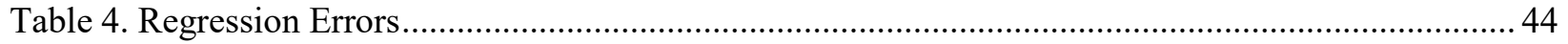

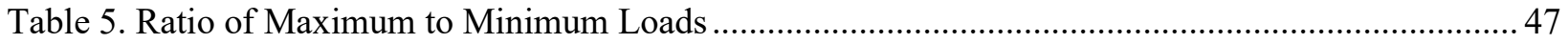

Table 6. Building a SARIMAX Model for Monday, Tuesday, Wednesday, and Thursday Using 20152018 Hourly Load and Temperature Data.............................................................................. 50

Table 7. Time Series Models Built Using Temperature and Load Data of Different Years Combination. 51

Table 8. Error Percentage Between Actual Load and Forecasted Load From Different Models ............... 51

Table 9. Effect of Household Energy Efficiency Measures on Overall Annual Energy Consumption...... 57 


\section{Overview of the Moroccan Electricity System}

Morocco relies on fossil fuels for electricity generation but is starting to use more renewable generation. Morocco intends to source at least $52 \%$ of total installed power capacity from renewable sources by 2030 . This goal was announced during the $21^{\text {st }}$ Session of the United Nations Framework Convention on Climate Change Conference of the Parties in 2015 ("Morocco Renewable Energy Target 2030 - Policies" 2019).

Fossil fuels accounted for over $80 \%$ of the country's electricity generation mix in 2017 . Wind accounted for 9\%, hydroelectricity 5\%, and solar 1\% ("Morocco - Countries \& Regions" 2020). Morocco imports $89.4 \%$ of its primary energy needs and $17 \%$ of its electricity needs (Bentaibi et al. 2019). Indigenous production of fossil fuels is negligible.

Renewable energy capacity has increased in dramatically in the past decade. Morocco currently has $1.22 \mathrm{GW}$ of wind capacity and 711 MW of solar capacity as of 2018 ("Morocco - Countries \& Regions" 2020). See Figure 1 for electricity generation by source.

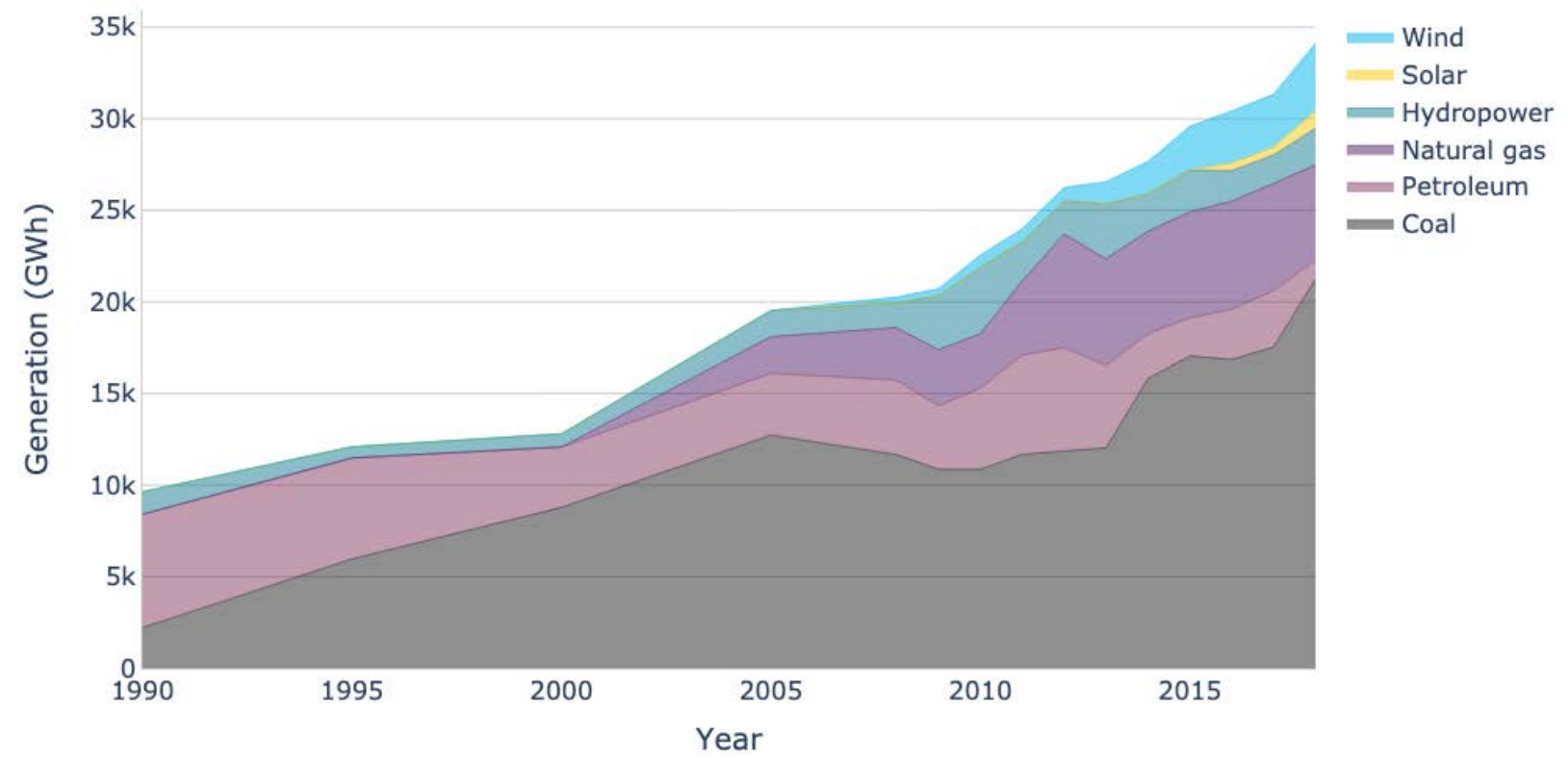

Figure 1. Electricity generation by source, Morocco 1990-2017

Source: Public data from ("Morocco - Countries \& Regions" 2020) and figures produced by NREL

Renewable energy production has increased in Morocco partly due to a regulatory push within the country. Morocco's National Energy Strategy of 2009 is organized around five pillars (Usman and Amegroud 2019):

- Optimize the fuel mix in the electricity sector

- Accelerate the development of renewable energy to reduce import dependency

- Make energy efficiency a national priority

- Encourage more foreign investment in the energy sector 
- Promote integration into the regional grids

Morocco's Renewable Energy Law 13-09 allows for the development of renewable energy and partially opens the electricity market to competition. Private entities may develop renewable energy generation projects and sell to large consumers. Private entities also are entitled to access to the power grid to transport electricity. The generators may also export electricity to Spain and Algeria (as Morocco has transmission connections to these grids). Later legislation allows for net metering for solar PV and onshore wind generation (Hochberg 2016).

Private generation constitutes about 50.8\% of total generation as of 2017 (Usman and Amegroud 2019). ONEE, the state-owned utility, previously had a monopoly on generation, transportation, and distribution of electricity.

Increases in renewable energy power generation are also due to the Morocco Solar Program. The Morocco Solar Program aims to increase solar generation through developing solar projects. The projects will include solar thermal, photovoltaic (PV), and concentrated solar power ("MoroccoSolar Program"). Currently, the Ouarzazate Solar Power Station, also called the Noor Power station, is the only solar power station in Morocco (REDE 2020). It is a 580-MW plant and is the largest concentrated solar plant in the world. The Moroccan Agency for Solar Energy signed power purchase agreements for the entire power output of the project ("Noor Ouarzazate Solar Complex, Morocco" n.d.).

Morocco has substantial solar energy potential, which can fuel a greater increase of renewable energy generation to meet both growing demands and Morocco's energy goals. Morocco receives over 3,000 hours of sunshine annually with an irradiation of approximately 5 $\mathrm{kWh} / \mathrm{m}^{2} /$ day, which was evaluated using NREL's Renewable Energy Data Explorer ("RE Explorer" 2021).

Morocco is estimated to have a $25 \mathrm{GW}$ of wind energy technical potential, and the government of Morocco seeks to develop this technical potential through the National Integrated WindPower Program (PNEI). PNEI aims to install 1 GW of wind energy by 2020 ("SIE" 2021). The wind potential is due to good climatic and geographic conditions for wind turbines, particularly on the coastline, where wind speeds can reach up to 10 meters per second (El Khchine et al. 2019) Additional geospatial analysis was completed by NREL for wind speed at 200 meters, which showed strong wind resources in the country ("RE Explorer" 2021). Both ONEE and private entities own wind farms in Morocco. The largest wind farm, Tarfaya, has a 300-MW nameplate capacity and is a joint venture of Nareva Holding and Engie (The Wind Power 2017). Nareva Holding also owns the second largest wind farm in the country, Midelt, with Enel GreenPower. Midelt has a 210-MW nameplate capacity (The Wind Power 2017). ONEE owns and operates the Tangier wind farm, which has a 107-MW nameplate capacity (The Wind Power 2017). Overall, of the 1,291MW nameplate capacity listed by Wind Power, ONEE owns and operates approximately $200 \mathrm{MW}$, about $15 \%$. The rest is owned and operated by private entities (The Wind Power 2017).

Morocco's electricity demand grew at an average rate of $6.5 \%$ annually from 2003 to 2014 and $3.1 \%$ between 2015 and 2019. This demand growth has been driven by economic growth and a growing industrial sector, which includes electricity intensive activities. High rates of 
electrification also contribute to growing electricity demand. In 1995, the country had $18 \%$ rural electrification. This has increased to $99.72 \%$ at the end of 2019 ("ONEE Data, Site Web Officiel de l'ONEE - Branche Electricité" 2021). Using tariff data, Figure 2 were generated to show the total annual consumption and percentage annual consumption by customer type. As can be seen, household electric load is the largest contributor to energy consumption in terms of GWh consumed. This suggests that household energy demand should be better understood through data collection or sampling to produce a standardized energy curve for load forecasting, followed by industrial energy consumption.

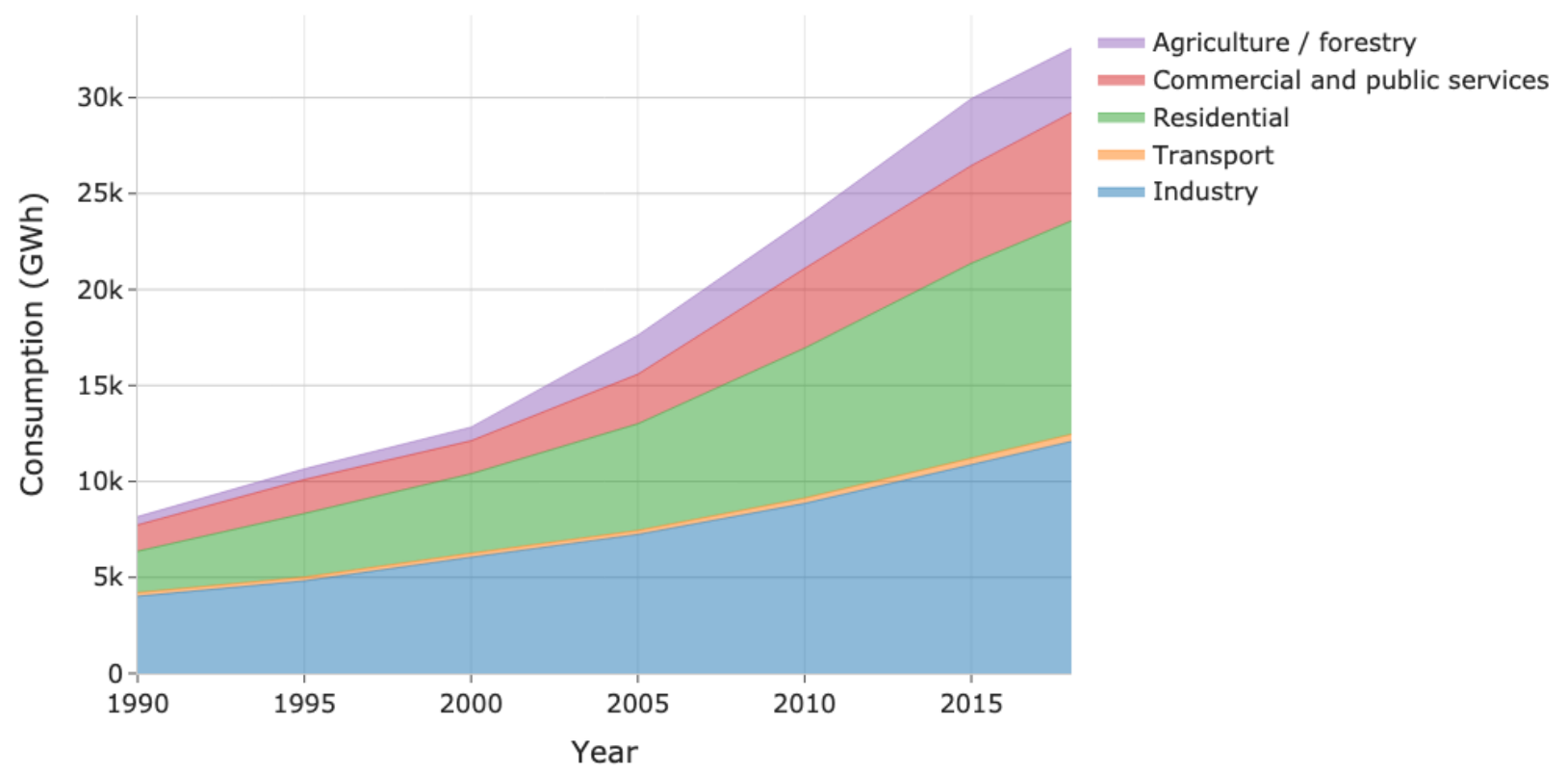

Figure 2. Annual energy consumption by sector

Source: Public data from ("Morocco - Countries \& Regions" 2020) and figures produced by NREL 
Table 1. Key Stakeholders in the Moroccan Electricity Sector

\begin{tabular}{|c|c|c|c|}
\hline Organization & Acronym & Type & Description \\
\hline $\begin{array}{l}\text { Moroccan Electricity and } \\
\text { Drinking Water Utility }\end{array}$ & ONEE & $\begin{array}{l}\text { Government } \\
\text { Utility }\end{array}$ & $\begin{array}{l}\text { The state-owned utility owns and operates the } \\
\text { transmission and a part of the distribution grid, } \\
\text { and it is the system operator and power } \\
\text { dispatcher. ONEE produces power generation } \\
\text { and transmission master plans. }\end{array}$ \\
\hline $\begin{array}{l}\text { Ministry of Energy, Mines } \\
\text { and Environment }\end{array}$ & MEME & Government & $\begin{array}{l}\text { The main authority over energy policy; it drafts } \\
\text { and enforces laws and regulations. }\end{array}$ \\
\hline $\begin{array}{l}\text { Energy Investment } \\
\text { Company }\end{array}$ & SIE & Government & $\begin{array}{l}\text { SIE supports the development of renewable } \\
\text { energy through investments. }\end{array}$ \\
\hline $\begin{array}{l}\text { Moroccan Electricity } \\
\text { Regulatory Authority }\end{array}$ & ANRE & Regulator & $\begin{array}{l}\text { Autonomous regulator that was established in } \\
2018 \text {. It regulates access to networks, sets } \\
\text { tariffs for utilization of transmission and medium- } \\
\text { voltage grid, and ensures efficient market } \\
\text { functions }\end{array}$ \\
\hline $\begin{array}{l}\text { Moroccan Agency for } \\
\text { Sustainable Energy }\end{array}$ & MASEN & $\begin{array}{l}\text { Private } \\
\text { company with } \\
\text { public } \\
\text { shareholding }\end{array}$ & $\begin{array}{l}\text { MASEN leads and manages the deployment of } \\
\text { renewable energy in Morocco. The goal is to } \\
\text { secure } 52 \% \text { of the country's energy mix from } \\
\text { renewable sources by } 2030 \text {. }\end{array}$ \\
\hline $\begin{array}{l}\text { Moroccan Agency for } \\
\text { Energy Efficiency }\end{array}$ & AMEE & Government & $\begin{array}{l}\text { AMEE has a mandate to focus on improving } \\
\text { energy efficiency in the usage and storage of } \\
\text { power. }\end{array}$ \\
\hline Distribution Companies & & Utilities & $\begin{array}{l}\text { There are } 11 \text { distribution companies ( } 7 \\
\text { municipal utilities and } 4 \text { private concessions). }\end{array}$ \\
\hline $\begin{array}{l}\text { Ministry of General Affairs } \\
\text { and Governance }\end{array}$ & MAGG & Government & $\begin{array}{l}\text { Responsible for price and competition policy, } \\
\text { also regulates electricity and fuel prices }\end{array}$ \\
\hline $\begin{array}{l}\text { Ministry of Economy, } \\
\text { Finance and } \\
\text { Administration Reform }\end{array}$ & MEFAR & Government & $\begin{array}{l}\text { Oversees the financial side of energy sector and } \\
\text { approves the investment plans of ONEE }\end{array}$ \\
\hline $\begin{array}{l}\text { Institute for Research into } \\
\text { Solar and Renewable } \\
\text { Energies }\end{array}$ & IRESEN & Government & $\begin{array}{l}\text { Identifies research priorities and projects and } \\
\text { disseminates research }\end{array}$ \\
\hline
\end{tabular}

Much of Morocco's electricity sector is controlled by government entities. There are, however, many public, private, and public-private organizations that support the energy supply. Table 1 highlights the key stakeholders within the Moroccan electricity sector. Figure 3 shows the relationships between these entities to provide context related to partnerships. 


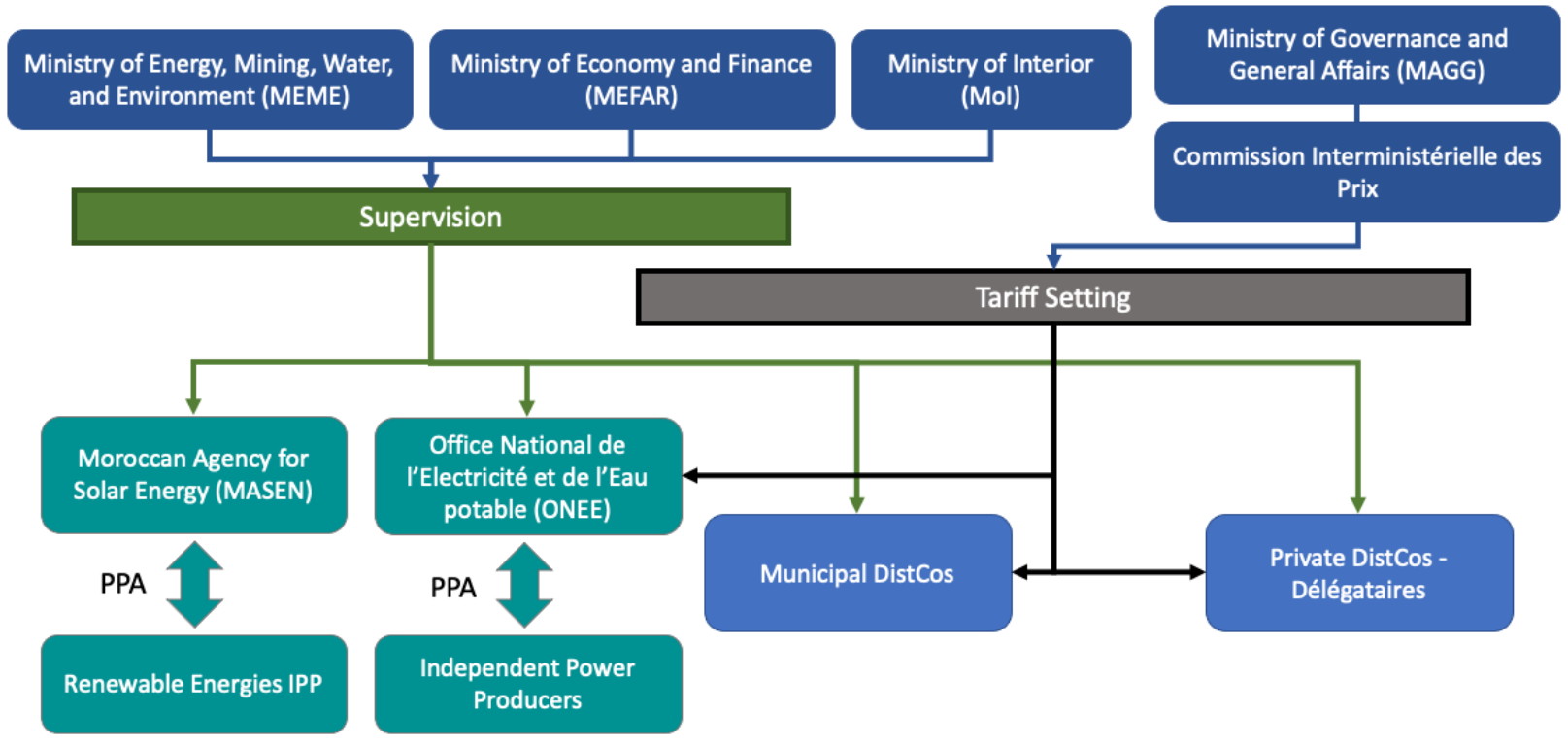

Figure 3. Private and public entities in the energy sector and their roles

Source: Produced by NREL, data from: (Usman and Amegroud 2019)

The Moroccan Solar Program involves many international stakeholders, as well as local stakeholders. MASEN was created in 2010 to implement the Program and SIE participates as a shareholder in MASEAN by owning $25 \%$ of shares and by participating as a member of MASEN supervisory board ("SIE" 2021). The Moroccan Solar Program is supported by the Moroccan government through supportive policies for renewable energy development, as discussed above. International financing comes from Climate Investment Funds, the World Bank, the Asian Development Bank, the European Investment Bank, KfW Group, and the French Development Agency. The program receives technical assistance from German Cooperation for International Collaboration (Senhaji 2016).

This information is provided for context and the load forecasting and planning under uncertainty sections that follow provide more detailed information related to future projected demand, integrating renewable energy technologies and energy efficiency for into grid systems. 


\section{Load Forecasting Introduction}

Ensuring the reliable and economic operation of complex power systems requires significant planning efforts from many different energy sector actors. These planning efforts have traditionally focused on key questions considered over a planning horizon, ideally including descriptions of key uncertainties and their impacts, such as:

- Load Forecast - What will the annual and peak electricity demand be?

- Electricity Generation Planning - How much and what type of generation is needed to serve this demand?

- Transmission Expansion Planning - What transmission system enhancements are necessary to ensure the reliable and economic delivery of electricity when and where it is needed (Katz and Milligan 2016)?

Load (or demand, or consumption) commonly refers to electrical energy (in GWh) and electrical power (in MW). Load forecasting is the process of predicting how a system's load may behave in the future over a given planning horizon. Forecasting is done for various horizons based on the purpose of the planning activity.

Energy policies and utility-scale integrated resource planning (i.e., processes for making generation, transmission, and sometimes distribution investment and retirement decisions) typically consider long-term (i.e., 10 or more years) planning horizons due to the significant lifetime of power system infrastructure, the timeline for infrastructure implementation, and the long runup needed for sector-wide energy transitions. Planning at this timescale requires longterm load forecasts. Short-term load forecasts (i.e., snapshot, day-ahead, or up to about 2 weeks in the future) are conducted to anticipate the dispatch of resources or for technical network studies. Medium-term load forecasts (i.e., months to years) are often completed for planning updates or nearer-term generation or system planning (Hong 2016).

Load forecasts are a key initial step in power system planning efforts and feed into subsequent analyses, as depicted in Figure 4. Developing a realistic, accurate forecast is critical to the development of a well-functioning power system. Load forecasts have traditionally laid the foundation for determining the least-cost mix of generation resources and transmission expansion options in power system planning. Under-forecasting load can result in system-wide generation shortages, which require load-shedding activities or expensive emergency generation. ${ }^{1}$ Alternately, over-forecasting load may result in an excessive financial burden on a utility, if a surplus of generation is planned and built, which may lead to higher prices for consumers.

To support these analyses, load forecasts generally result in the following three components:

1. Energy consumption (in GWh or MWh)

2. Peak demand (in GW or MW)

3. Hourly load profiles for a typical year ( 8,760 hours) or in time slices that capture seasonal, weekly, and daily variations (IRENA 2017; Carvallo et al. 2016; Herrick 2016).

\footnotetext{
${ }^{1}$ See (Hale et al.) for a case study in South Africa that shows a demand response program quickly enacted.
} 
Wherever the data allow each of these components should have temporal and spatial resolutions to support planning efforts. They may be disaggregated by sector (e.g., residential, commercial, industrial, and public). Temporal resolution refers to the number and/or length of time slices represented by the data (e.g., annual, monthly, hourly, and subhourly). Spatial resolution refers to the number of data points within the geographic area of study, crucial for transmission planning, especially with large quantities of variable renewable generation (Cox et al. 2018).

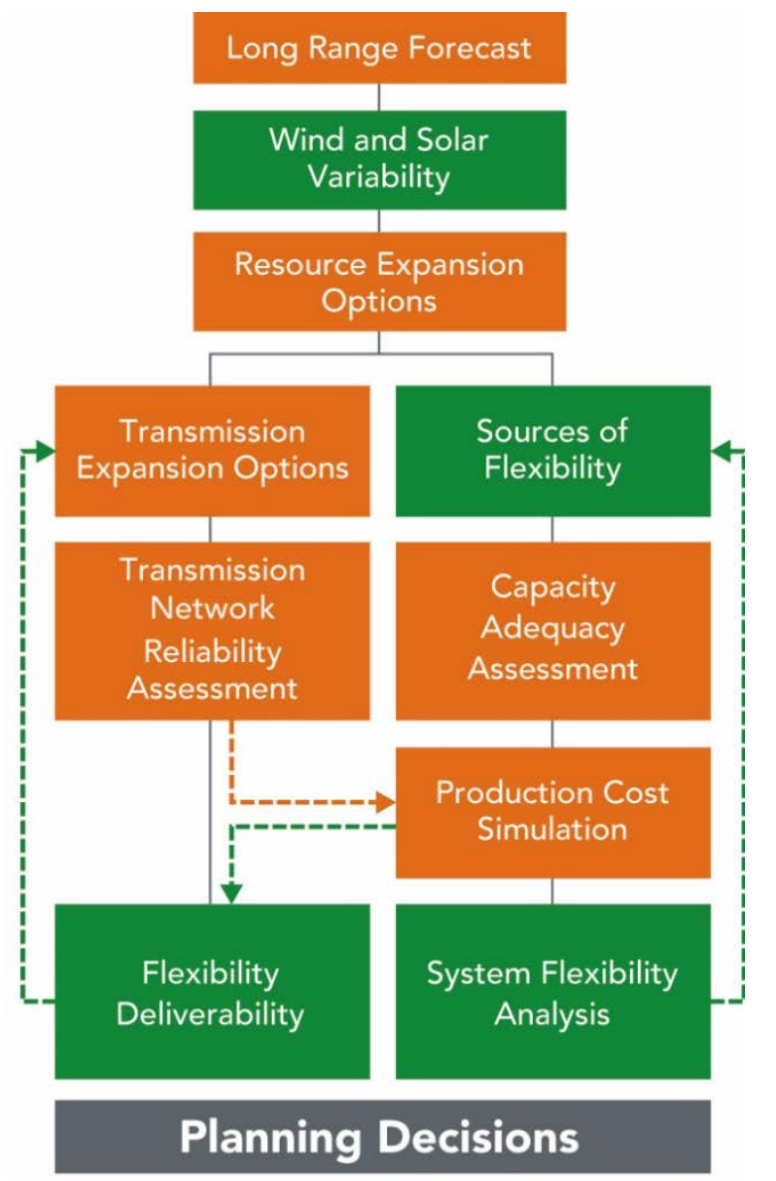

Figure 4. Key steps of traditional power system planning processes (orange boxes) and additional steps for integrating variable renewable energy (green boxes)

Source: (Katz and Milligan 2016)

Load forecasting is a challenging process for all utilities, but is made especially difficult in developing economies, where the required data are often less readily available and rapid changes in sectoral demand are common. Many developing economies are seeing high gross domestic product (GDP) growth and are forecast to continue that trajectory. Developed economies often utilize inexpensive labor and moderate regulations to provide supply chain inputs more cheaply. This industrial, and less agricultural, activity is sometimes electricity-intensive, causing the forecast demand for power to be aggressive, critical, and challenging to supply.

Disruptive technologies, such as distributed generation, can confound load forecasting for developing economies. Power sector projections can embed an estimate of cost projections and adoption forecasts for things like rooftop solar, small-scale energy storage, and electric vehicles. Further, it is sometimes difficult to determine where the country lies on the electricity intensity 
curve. Even if efficiency best practices are implemented (e.g., less electric load per appliance), plug load may increase due to an increase in the number of devices, with former "luxury" items becoming affordable to more people.

With energy efficiency and other factors, load growth is no longer a certainty in some countries. For example, areas of the United States are forecasting flat or even declining load growth, from distributed generation, energy efficiency, and electricity demand being less linked to gross domestic product (due to more service-based economies). In the next decade, three major U.S. regional transmission organizations are forecasting flat or slightly declining load $(-0.6 \%$ for the New York Independent System Operator, $-0.14 \%$ for ISO New England, and $0.4 \%$ for the PJM Interconnections) (Walton 2018).

While developing economies are not forecasting declining demand, load forecasting is difficult and inherently includes significant uncertainty. The techniques discussed within this paper are some considerations to mitigate these uncertainties and more anticipate future load. This document summarizes common approaches, including tools and skill sets, that are helpful for load forecasting, as well as case examples of load forecasting in select countries. A detailed outlook for Morocco follows, including expected load growth and discussion around possible improvements for future load forecasts. 


\section{Load Forecasting Methodologies}

At its most basic, load forecasting methodologies attempt to estimate load at some time in the future $\left(\operatorname{Load}_{t+1}\right)$ based on information on current (or historical) load $\left(\operatorname{Load}_{t}\right)$ and some assumptions of how this load may evolve in the future $(C)$ as depicted as:

$$
\operatorname{Load}_{t+1}=C \times \operatorname{Load}_{t}
$$

Here, $C$ may represent many different values or additional equations, where:

- In its simplest form, $C$ is a constant estimated by an expert, the forecast is a judgmental approach that uses extrapolation to project load into the future. For example, $C$ could be as simple as a constant expected load growth rate-such as $5 \%$, where $C=1.05$.

- $\quad C$ is determined through historical time series analyses or dependent and independent variables that affect load. The model may be a time-series analysis or an econometric approach.

- The information contained in $C$ is based on studies of the share of end-use technologies and their energy intensities. The model may be an end-use or engineering model (Bhattacharyya and Timilsina 2009).

In reality, the last two approaches yield the estimate of load in the future $\left(\operatorname{Load}_{t+1}\right)$ based on a function of historic load $\left(\mathrm{f}\left(\operatorname{Load}_{t}\right)\right)$ :

$$
\operatorname{Load}_{t+1}=\mathrm{f}\left(\operatorname{Load}_{t}\right)
$$

In many cases, multiple future load data points are being forecast from multiple past load data points. Regardless, each of these approaches is distinct from one another and may have varying degrees of complexity, which may require different user skill sets. The methodologies highlighted in this report include time-series analysis, econometric, and end-use approaches.

In general, there are independent variables on which the dependent variable, electricity generation, relies. Independent variables, which commonly can help predict electricity consumption, include:

- GDP

- Price of power (both current and historic)

- Population and population growth

- Household size

- Weather factors

- Individual customer sector growth (e.g., industrial, commercial, domestic, agriculture, bulk supply, public lighting)

- Employment (or other labor statistic)

- Demand-side technology adoption (e.g., air conditioning, refrigeration, electric vehicles).

Regardless of which method is used, having historically complete data, and the most accurate forecasted set of independent variables, is beneficial to the robustness and fidelity of load forecasts. While a large part of the load forecasting work involves collecting and providing quality control of electricity generation numbers, it is typically other governmental organizations who are tasked with data veracity for these other demographic or economic variables. 


\subsection{Time-Series Analysis Approaches}

Time-series analysis assumes a future trend based on the past data (or the impact of parametric changes on load growth) and accounts for correlations with diurnal cycles, weather, and other cyclical phenomena. This form of analysis was the approach of choice during the mid-20th century (Weron 2006; Singh and Khatoon 2012). It requires the smallest volume of data to inform the analysis: weather data and historical electricity load data. These time-series methods are limited to time-based data and statistical treatment of that data, and initially were primarily based on regression.

This approach can be useful for simple load trajectories and does not require powerful data analytics, but it does not capture the complexity and "lumpiness" of real-world economic systems and technological adoptions, such as air conditioning. Further, it produces inaccurate results if the underlying structure of the data changes, as with a shift from an energy-intensive production economy to a more energy-efficient service economy, as well as growth in selfgeneration options. As described in Section 3.2, more advanced statistical and machine learning methods can be applied, incorporating more variables such as temperature data with autoregressive algorithms.

Auto regressive models are one class of time-series model. In simple auto regressive models, past values of the load $\left(L_{t-i}\right)$ are used to forecast the future load $\left(L_{t}\right)$. In the following equation, $C$ is a constant, $\emptyset_{1}, \ldots \varnothing_{p}$ are coefficients of the lag demands, and $\epsilon_{t}$ is the forecasting error. The order of the model indicates the number of lags $(p)$ that are included (Bozkurt et al.).

$$
L_{t}=C+\emptyset_{1} L_{t-1}+\emptyset_{2} L_{t-2}+. . \emptyset_{p} L_{t-p}+\epsilon_{t}
$$

Time series models such as the auto regressive moving average (ARMA) and seasonal auto regressive integrated moving average (SARIMA) use past values of load in terms of lag demands and lag values of the errors. The moving average component of the model tells dependency of the load $\left(L_{t}\right)$ on the lagged forecasted errors $\left(\epsilon_{t-j}\right)$. Combining both auto regressive and moving average models, the ARMA model can be defined as the following equation, in which $\theta_{1}, \ldots \theta_{q}$ are the coefficients lag forecast errors included up to q numbers.

$$
L_{t}=C+\emptyset_{1} L_{t-1}+. .+\emptyset_{p} L_{t-p}+\epsilon_{t}+\theta_{1} \epsilon_{t-1}+\theta_{2} \epsilon_{t-2}+. .+\theta_{q} \epsilon_{t-q}
$$

Furthermore, in auto regressive integrated moving average (ARIMA) models time series data needs to be differenced to make the data series stationary. Generally, the ARIMA model is defined as the $\operatorname{ARIMA}(p, d, q)$ model, where $p$ is the number of autoregressive terms, $q$ is the number of moving average terms, and $d$ is the number of nonseasonal differences needed for stationarity of data. The $\operatorname{SARIMA}(p, d, q) \mathrm{x}(P, D, Q)$ model for seasonal patterns, which is daily for Morocco electricity demand forecast, is an extended version of ARIMA with additional seasonal terms, where $P$ is the degree of seasonal, $Q$ is the degree of seasonal moving average, and $D$ is the degree of seasonal integration.

In addition to the past load values, there are other variables that influence the short-term electricity demand (Rafal Weron). Specifically, weather is an important factor that can be included as an exogenous input to the time series model. In the seasonal auto regressive 
integrated moving average with exogenous variable (SARIMAX) model (see the following equations), electricity demand $\left(L_{t}\right)$ is forecasted using demand of previous time steps $\left(L_{t-i}\right)$ with coefficients $\left(\emptyset_{i}\right)$, exogenous variable $\left(W_{k}\right)$ with coefficients $\left(\omega_{k}\right)$. Moving average window for error terms $\left(\epsilon_{t-j}\right)$ with coefficients $\left(\theta_{j}\right)$ and constant $(C)$ are also included in the model (Bozkurt, Biricik, and Tayşi 2017; Bennett, Stewart, and Lu 2014).

$$
L_{t}=C+\sum_{i=1}^{p} \emptyset_{i} L_{t-i}+\sum_{j=1}^{q} \theta_{j} \epsilon_{t-j}+\sum_{k=1}^{r} \omega_{k} W_{k}+\epsilon_{t}
$$

This analysis requires staff with a background in data analytics and analysis tools such Microsoft Excel, MATLAB, Python, or R. For example, and as will be discussed in Section 9.4, Python provides an ARIMA model for time-series forecasting.

\subsection{Machine Learning Approaches}

Machine learning methods have been extensively used for load forecasting because of the strength of these techniques in modeling nonlinearities. Among several machine learning methods, support vector machine, artificial neural networks, fuzzy logic, and evolutionary algorithms have been applied for several studies. Support vector machine is a class of supervised learning method, which transforms nonlinear load forecasts into high dimensional space, and then uses linear regression in the new space (Cortes and Vapnik n.d.; Turkay and Demren 2011).

Artificial neural network is computational model that is inspired by the way biological neural networks in the human brain process information. In the artificial neural network models, nature of connecting input and output is not predetermined, and optimal structure of the model is identified through the training process. The basic unit of computation in a neural network is the neuron, often called a node or unit. It receives input from some other nodes, or from an external source and computes an output. Each input has an associated weight (w), which is assigned based on its relative importance to other inputs. During the training process these weights are adjusted iteratively. There are different types of artificial neural network, and different options of selecting the activation functions, layers and neurons of the model (Houimli, Zmami, and BenSalha 2020).

\subsection{Econometric Approaches}

One approach for long-term load forecasting that incorporates additional complexity is econometric modeling, which may be combined with time series and end-use approaches (Feinberg and Genethliou 2005). These are statistical analysis methods that rely on large data sets to develop regression models that can predict future energy demand, or load. These models can also account for feedback loops within the system, meaning changes in one variable, such as number of customers, could impact other variables, like utility tariffs. They also can account for uncertainty in future changes in these variables by incorporating a stochastic (or random) element to the analysis. This can be useful to show the sensitivity of the load projection to changes in certain variables.

Econometric modeling approaches predominate among Organization for Economic Cooperation and Development country utilities and incorporate multiple drivers of energy demand, such as 
economic activity (GDP), weather, electricity prices, and natural gas prices. This modeling approach makes basic assumptions about the relationships between energy demand and these drivers; thus, it can account for the impact of competing fuels or generation technologies. It is critical to note that if these underlying assumptions change, such as the GDP to electricity use relationship changing because of a transition to a less energy-intensive service economy, econometric models can be very inaccurate. Accurately representing the structure of the economy in the future is an extremely important part of econometric modeling that can be difficult to do during times of transition. However, an advantage of this approach is that it may be easier to gather data on these economic drivers than the customer-specific information required for an end-use model.

Utilities will likely need staff with a deeper background in statistical analysis and econometrics to perform this type of analysis. Econometricians usually divide their attention between economic theory, scientific study, and statistical analysis. Some potential software tools include the MATLAB econometrics toolbox, R, STATA, Eviews, SPSS, Microsoft Excel, and Python.

Another approach, in lieu of the models discussed above, is the use of computer learning algorithms. This approach applies an artificial intelligence algorithm that will often produce high accuracy models that can handle the complex relationships that exist in the power sector. The downside of these models is that they can involve complicated, unintuitive modeling equations that are not generalizable beyond the data set they used to develop the model and require vast quantities of training data. Some of the most common artificial intelligence-based methods include genetic algorithms, support vector machine, and fuzzy logic models (Mosad 2015; Singh and Chaturvedi 2013). These models are commonly available in statistical packages that can be used in such major software as Microsoft Excel, R, MATLAB, SAS, Python, and Julia, among many others, and likely require staff with degrees and experience in data or computer science.

\subsection{End-Use Approaches}

End-use models employ a "bottom-up" or "engineering" approach, and generally forecast load from the end-user standpoint. This method can use various levels of detail. At the most detailed, the bottom-up approach uses full data sets of the existing number of customers and their estimated electric load by end use. Bottom-up approaches can account for the impact of customer behavior, such as distributed technology adoption or participation in demand-side management programs (Gotham 2007). Although this approach can be very accurate, it is highly dependent on good quality end-use data that can be hard to obtain from customers, such as detailed customer load profiles and appliance specifications. Due its data intensity, this approach can also be combined with simpler methods. For instance, a bottom-up approach may capture some detailed end uses where data is available and then estimate the rest of the load with a simpler approach (Seiden and Eakin 2017; Fay and Ringwood 2010).

Utilities will likely need diverse engineering skillsets to accurately model the energy consumption of different sectors, as well as data analysis skills to manage the large volume of data required for these estimates. The International Atomic Energy Agency has developed a range of tools to assist developing countries with end-use modeling, including the Model for Analysis of Energy Demand (MAED) and the Wien Automatic System Planning Package, which is one of the most widely used models in developing countries for energy system planning. In addition, the Long-range Energy Alternatives Planning System (LEAP) tool, developed at the 
Stockholm Environment Institute, is widely used around the world, and can be utilized for enduse modeling (IAEA 2018). 


\section{Evolving Methods of Load Forecasting}

Many countries are undergoing rapid structural changes to their energy markets, including increases in energy efficiency standards, tariff increases due to infrastructure investment, and growth in distributed generation technologies (Seiden and Eakin 2017). As a result, load forecasting has become an especially challenging exercise in recent years. To decrease the risk of overbuilding unneeded capacity, utilities are adopting several novel approaches to load forecasting.

\subsection{Planning Horizons}

Load forecasting can take place on several timescales. These timescales are meant to match decision-making. As described above, long-term load forecasting is often considered as 10 or more years, with short-term load forecasting on the order of days or weeks, and medium-term load forecasting filling the gap (Hong 2016; Singh and Khatoon 2012). The timescale is chosen based on the needs of the entity making decisions. For example, long-term load forecasting can be used to produce integrated resource plans charting out a path for the energy system over decades. Additionally, large projects such as coal, nuclear, and transmission infrastructure are served by long-term load forecasting. Short-term load forecasting assists in the day-to-day operations of the electric grid and planning the next several hours or days. Medium-term forecasting can help with planning operations, such as which season plants should go offline for maintenance, or when to build projects with shorter lead times, such as solar PV or natural gas plants. Different timescales are used for different decisions support and should, therefore, be matched based on the needs of the utility.

\subsection{Load Forecast Methodologies}

A study of the load forecasts of 12 integrated resource plans from load-serving entities in the United States found that forecasts with more sophisticated approaches had less forecast error than others that employed simpler approaches (Carvallo et al. 2016). This finding suggests that there may be benefits to the planning process from increased model complexity. As discussed above, this model complexity requires increased data input in addition to a higher skill level for modelers.

A shift toward end-use load forecasting may be of use where the required data are available as well as capable modelers. In a publication in Public Utilities Fortnightly, Seiden and Eakin (2017) outline a bottom-up load forecasting approach that incorporates utility grid data, thirdparty customer data, as well as the traditional variables such as economic, population, and weather data (Fay and Ringwood 2010). This approach requires a very large amount of data but may yield valuable location-specific load forecasting insights that were previously impossible with older computing technology.

\subsection{Ensuring Resource Adequacy}

Generation capacity markets play a pivotal role in balancing supply and demand and therefore require accurate load forecasts. Some new approaches to capacity procurement allow flexibility by the utility or operator such that they can revise medium-term forecasts more frequently. For example, PJM, a regional transmission operator coordinating wholesale electricity dispatch in 13 states in the United States, has adopted an approach called incremental auctions, which can be 
conducted 3 and 5 years out, allowing utilities to correct inaccuracies in load forecasting (Walton 2018). However, this approach is only appropriate if generation technologies with shorter building lead-times are economically competitive. This approach allows opportunities for planners to balance out supply and demand in the medium term, but it also requires a consistent cycle of completing load forecasts, evaluation of how results compare with actual demand, and revision of load forecasts at certain decision points.

\subsection{Temporal and Spatial Resolution of Load Forecasts}

With increased penetrations of variable renewable energy (VRE) generation on the grid, planners and system operators must address the variable, uncertain, and geographically dispersed nature of these resources. Higher temporal and spatial resolutions become necessary to better match provision of geographically dispersed supply with geographically dispersed demand in the context of generation and transmission planning.

\subsubsection{Temporal Resolution}

Temporal resolution refers to the number and/or length of time slices represented in the data (e.g., annual, monthly, hourly, and subhourly). Long-term power system planning often defines two levels of temporal resolution. First is the resolution, or frequency, of modeled capital investment decisions for generation and transmission. The planning horizon often covers large portions of power plant and other power system infrastructure lifetimes (15-40 plus years) that is resolved into a number of investment decision points (every 1-5 years). Second is a finer resolution sufficient to describe operational characteristics of the power system. These would include, for example, the variability of the system's load, VRE supply resource variability, as well as the flexibility requirements and technical characteristics (ramp rate limits and minimum generation levels) of the dispatchable power plants (IRENA 2017).

Higher temporal resolution (hourly or subhourly) load forecasting enables planners to better estimate appropriate operational parameters by capturing the temporal match between demand and the availability of VRE. Annual demand is typically broken down into hourly slices to represent the variability of demand over 8,760 hours in a year in order to capture the seasonal, weekly, and daily variations of load. An hourly or subhourly load depiction that can be synchronized with daily and seasonal variations in VRE availability allows for alignment of VRE generation with demand, allowing planners to identify the contribution that VRE can make to firm capacity (aggregate capacity that is assured to meet demand) (IRENA 2017). Assigning capacity credits (or the percentage of nameplate capacity attributable as firm capacity) to all generation resources allows planners to employ generation expansion models to ensure sufficient firm capacity through the planning horizon. ${ }^{2}$ Without high-resolution load data, the variability of supply and demand would not be synchronized, possibly leading to suboptimal or inadequate capacity mixes as generation expansion models would not accurately represent periods of overor under-generation. Additionally, any flexibility needs in the system such as ramping capacity may not be accurately captured. High-resolution data on VRE resources such as wind and solar have become more readily available to support these analyses (IRENA 2017).

\footnotetext{
${ }^{2}$ Many utilities and load-serving entities use a capacity credit approach to estimate capacity needs, but increasingly use probabilistic analysis (rather than a capacity credit estimation) to ensure sufficient resource adequacy (Frew et al. 2019).
} 


\subsubsection{Spatial Resolution}

Spatial resolution refers to the number of data points within the geographic area of study (e.g., a $1 \mathrm{~km} \times 1 \mathrm{~km}$ grid of cells over a continuous area, discrete nodes, or defined geographic area) (Cox et al. 2018). An increased spatial resolution in load forecasts is an important consideration in long-term generation planning (IRENA 2017).

VRE generation, such as wind- and solar-based generation, are typically located in windy and sunny areas, which may be distant from load centers or existing transmission systems. Like large hydropower, the grid must be extended to these energy resources, as they are location constrained. Alternatively, lower-class wind, solar, and hydro resources may be available close to existing grid infrastructure and transmission capacity, which may provide a lower life cycle cost if new transmission infrastructure is not needed or can be developed more readily. Ensuring models can represent forecasted load by multiple nodes (location where demand is present) in the region of study can help estimate the potential cost of transmission expansion to connect VRE resources to load (IRENA 2017).

Increased spatial resolution of on- and off-grid load can also support planning activities focused on increasing energy access through grid extension or off-grid energy systems. Locations with forecasted load can be compared to spatial data on VRE resources and transmission systems (Khatib, Mohamed, and Sopian 2013; Palit and Chaurey 2011; Sharif and Mithila 2013). With off-grid cases, there is increased necessity on understanding both the uncertainty of predicting VRE output and the load forecast uncertainty. There are several methodologies tailored to this work that are also applicable to load forecasting writ large (Khatib, Mohamed, and Sopian 2013). 


\section{Case Studies of Long-Term Load Forecasting in Select Developing Countries}

Load forecasting is in iterative process that relies heavily on past experiences and learning from others best practices. As such, presented here are case studies from other countries to provide more background and best practices on load forecasting globally.

As part of a study by the World Bank, an analysis of the historical accuracy of the demand forecasting of 106 developing countries, from 1960 to 2012, was performed. This study concluded that "econometric forecasts are highly accurate for most of these countries." It also noted that the forecast accuracy of econometric models was significantly higher than that of simple heuristic models like assuming a fixed growth rate. Econometric forecast accuracy was diminished in countries where "rapid economic and structural transformation or exposure to conflicts and environmental disasters makes it difficult to establish stable historical demand trends" (Steinbuks 2017). Below are two case studies from countries successfully establishing their load forecasting practices based on global best practices.

\subsection{Colombia Case Study}

In Colombia, load forecasting is performed by the National Mining and Energy Planning Unit (UPME), which is a Special Administrative Unit attached to the Ministry of Mines and Energy. UPME must establish the energy requirements of the population and economic agents of the country as bases for its demand forecasts, taking into account the most likely evolution of demographic and economic variables and prices of energy resources, and prepare a National Energy Plan and the Electric Sector Expansion Plan in coordination with the National Development Plan (Ministerio de Minas y Energía and Unidad de Planeación Minero Energética - UPME 2018).

UPME releases annual revisions of Colombia's plan to expand generation resources and transmission networks, based on a mix of past data and projections. The analyzed data include types of electricity generation used in Colombia and their proportion, installed power capacity, and peak load. Individual projections include the growth of each type of generation, fossil fuel prices, regional electricity demand, and total electricity demand growth.

For projection of national electricity demand, UPME employs a combination of econometric forecasts along with multivariate models such as the Autoregressive Vector Model (for vector auto regression, or VAR) and the Model of Error Correction Vectors (for vector error correction). The inputs of this load forecast are historical energy demand in Colombia as reported by XM, the national system operator, macroeconomic data (total GDP) from the National Administrative Department of Statistics, demographic data from the United Nations (UN), and climatic data from the Institute of Hydrology, Meteorology, and Environmental Studies. After the econometric data (historical electricity demand, GDP, population, and temperature) are fed into the multivariate models, UPME combines the resulting series into a comprehensive forecast.

To address factors outside the econometric variables, UPME must consider external influences on future energy usage. In Colombia these include increased electric vehicle presence and a government initiative to promote public transit. Along with usage projections for Colombia's 
largest private-sector consumers of electricity, these factors are used by UPME to guide electricity demand projections. The results are low, medium, and high usage scenarios that represent the boundaries for Colombia's forecasted load. This forecast is used by UPME, the Regulatory Commission of Energy and Gas, the Ministry of Transport, and Ministry of Environment and Sustainable Development to plan and approve expansion of generation capacity, transmission infrastructure, and distribution lines.

\subsection{Philippines Case Study}

The Philippines Electric Power Industry Reform Act of 2001 established policy objectives aimed at restructuring the sector to improve power system reliability and reduce electricity tariffs through power market competition. This has introduced several new players to the power sector, as the sector moved from a vertical to a horizontal model. With the addition of the Renewable Energy Act of 2008, the Philippines has set a course to achieving energy security and clean energy generation in the country. Achieving these objectives requires strong power sector planning activities that allow the many different actors (generation companies, transmission system operators, distribution system operators, among others) to thrive.

The Philippines Department of Energy (PDOE) considers the preparation of an accurate load forecast a crucial first step of power sector planning and a key initial input to subsequent steps as this will determine the country's future power requirements and the necessary investments in generation, transmission and distribution to ensure demand is balanced with supply (PDOE 2017). The PDOE provides a national long-term load forecast used in national power sector planning efforts such as the Philippine Energy Plan, Power Development Plan, and Transmission Development Plan. As in many power sectors, individual actors also do some independent load forecasting for their own planning and/or comparison with the official forecast.

Load in the Philippine Energy Plan is forecast for different sectors (e.g., industry, residential, commercial, and agriculture, fishery, and forestry sectors). Energy demand, in MW, is projected using the Simple Econometric Simulation Systems, Expanded software, which is an econometric simulation tool add-on application for Microsoft Excel (Yamaguchi 2015; PDOE 2018). To refine the load forecasts from Simple Econometric Simulation Systems, Expanded, PDOE considers how sectoral road maps (e.g., residential and industrial) and other relevant information possibly not captured in the econometric simulation could also affect future demand. Philippine Energy Plan Energy consumption (GWh) forecasts are developed based on the peak demand projections developed for the Philippine Power Development Plan. The load forecast information is combined with least-cost generation system expansion plans to meet demand. Demand is separately developed in the Model for Energy Supply Strategies and their General Environmental Impacts tool from the International Atomic Energy Agency. The LEAP model from the Stockholm Environment Institute was used to consolidate the models into a national energy outlook.

The peak demand forecast for the Philippine Power Development Plan relies on a substantial set of data inputs and participation from power sector actors, as shown in Figure 5. Private utilities, electric cooperatives, nonutility customers (e.g., those with high enough electricity requirements to purchase directly from the transmission system), new industry customers, and generators all submit data and plans to allow for a power market assessment. This assessment produces the data 
inputs, such as historical electricity sales, peak loads, and utility forecasts, to forecast peak demand for the system along the planning horizon.

Forecasting future electricity sales is the first step in forecasting peak demand for the system. The baseline electricity sales forecast is calculated with a projection of GDP from the national Development Budget Coordination Committee and calculated historical trends of energy intensity (i.e., electricity consumption per unit of GDP or GWh/unit GDP), which are used to estimate energy intensity elasticities. Historical consumption values considered to be outliers resulting from extreme weather events or financial crises are not used to calculate energy intensity and elasticities. Gross generation, in GWh requirements are then forecast by adding in losses, such as transmission and distribution losses, and generator own-use requirements. A load factor $(\%)$, which represents the ratio of the forecast demand inMWh to the peak demand in MW, is then applied to calculate peak demand. This load factor is a fixed value based on the base year calculated load factor.

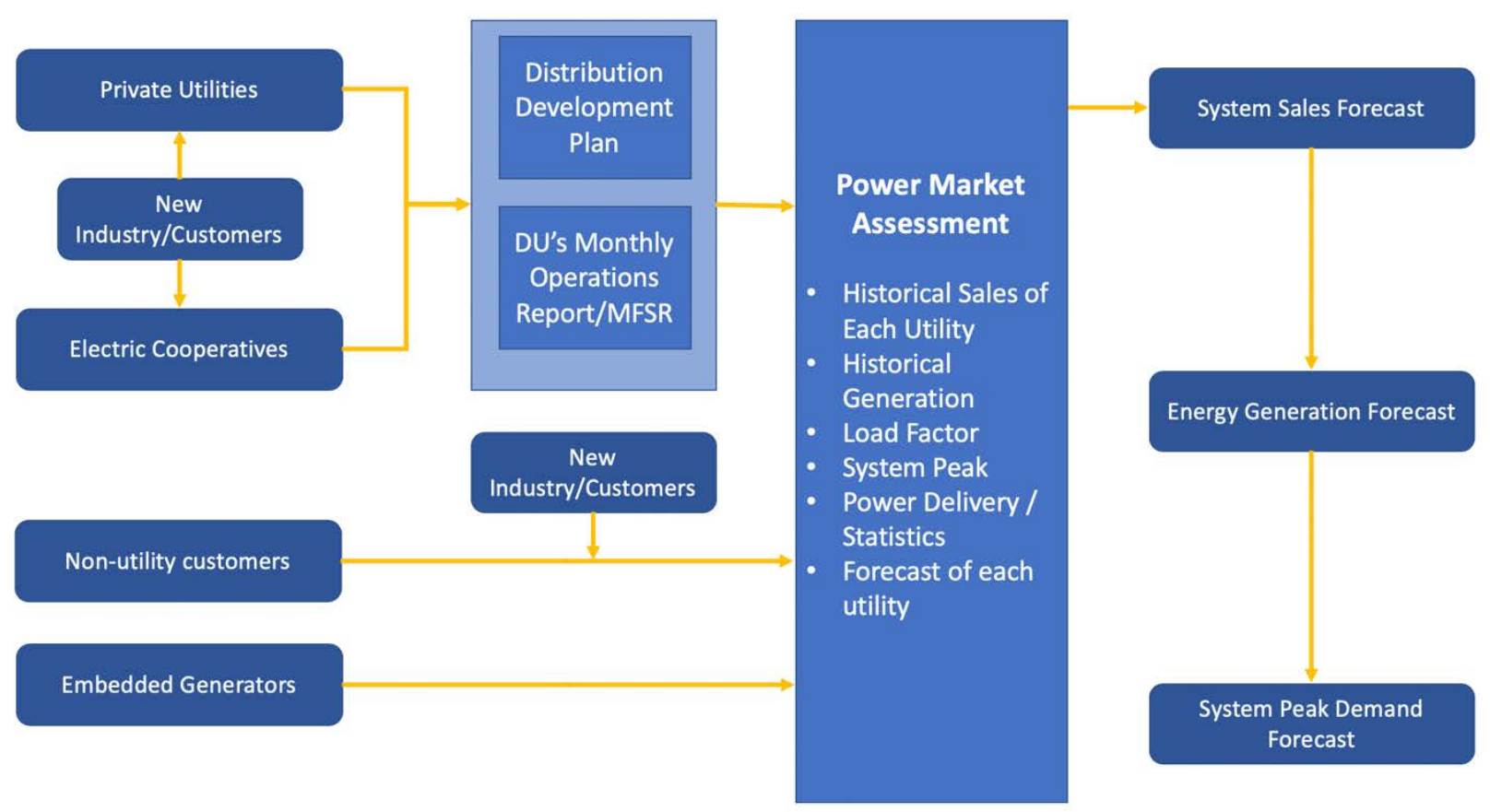

Figure 5. Peak demand forecast approach for the Philippine Power Development Plan

Source: (PDOE 2018)

The long-term load forecast from the PDOE is also used to support the development of the country's Transmission Development Plan. The National Grid Corporation of the Philippines (NGCP) is the transmission system operator in the country and is tasked with the development of the Transmission Development Plan, which sets the 25-year road maps of the expansion of the power grid. The Transmission Development Plan development begins with the submission of the long-term load forecast and the generation capacity additional alignment from the PDOE to NGCP, which are updated annually by the PDOE. NGCP then breaks down the forecast into individual transformer loads and noncoincident substation peak load forecasts to support substation extension planning needs (NGCP 2018). 


\section{Dealing With Uncertainties}

Any load forecast requires multiple input parameters, which are frequently dependent on additional parameters that are subject to many future uncertainties. This is further complicated by the uncertainties that exist with the large number of energy sector players in both regulated and unregulated environments. Parameters that face uncertainty, and may affect load in the future, are very context-specific, and include (Seifi and Sepasian 2011):

- Economic growth (or decline)

- Population growth (or decline)

- Inflation, depreciation, interest rates, and other economic parameters

- Fuel costs and electricity prices

- Technological development, including electric mobility options and distributed generation such as rooftop solar PV

- Regulatory and legal acts

- Costs for investment, operation, and maintenance of energy systems

- Resource availability

- Conflicts

- Unmet/suppressed demand levels

- Electricity access rates

- Social factors.

Add to this list more recent developments such as the decreased cost of behind-the-meter generation and increased electric vehicles, and it becomes obvious that accounting for all potential uncertainties in a single load forecast is nearly impossible. This means that there will never be a completely accurate load forecast. The purpose of these forecasts is therefore to provide information to decision makers and estimate the impact of different technologies and policies on future system loads. While load forecasting methodologies are never perfect, there are ways to reduce the uncertainty of these forecasts.

\subsection{Scenario-Based Approaches}

Forecasting a single future load does not provide sufficient information to decision makers about all the potential future trajectories of load. Considering multiple forecasts allows for more exploration of different possible futures. There are two common ways to construct multiple related projections:

- In a scenario-based approach, each scenario is built on parameters that are not within the control of the planner but are relevant to future situations, such as economic growth, industrial growth, or population growth.

- An alternative or an option, on the other hand, describes the impacts of policies or other interventions that a planner may control.

Multiple scenarios can be built that consider different potential futures of concern and planners can then see how load forecasts and generation expansion alternatives may change in these different scenarios (Bhattacharyya and Timilsina 2009; Seifi and Sepasian 2011). 
As the population growth, economic development path, or other parameters in many developing countries may be uncertain, consideration of multiple scenarios can help to understand how load forecasts may vary. Developing multiple load forecasts allows for planners to see what generation expansion, transmission system, and even demand-side management alternatives may be the most robust across multiple possible future pathways. Planners can also identify decision points along the power system planning process at which different alternatives may be more advantageous for potential scenarios. Many analytical techniques exist to measure the robustness of plans and may be scenario-based or probabilistic (i.e., stochastic) risk-based assessments (Carvallo et al. 2016).

Part of scenario-based load forecasting is sensitivity analysis. Some inputs of the chosen load forecast methodology may have a greater effect on the resulting forecasts than other inputs. Conducting a load forecast sensitivity analysis can help to develop long-term strategies, such as integrated resource plans, that can pivot allowing for the plan to adjust to different futures, similar to those that consider multiple scenarios. Planners can understand the effect and the magnitude of this effect on load forecasts for different inputs, such as population, prices, or economic growth, and understand what plan may be best adapted to the load forecast and adapt as new information is gathered in real time (Carvallo et al. 2016).

\subsection{Probabilistic Methods}

Probabilistic load forecasting (PLF) methods are augmentations to point forecasting methods. Where point forecasting methods produce a single value often coupled to some uncertainty, probabilistic methods often provide a distribution of likely values based both on previous electricity loads and on different probability estimates for likely events. For example, as VRE has expanded, probabilistic methods can help encapsulate the likelihood of different VRE outputs using past weather data. Additionally, probabilistic methods can incorporate other uncertainties such as system or generator failure into load forecasts. PLF includes a probability at each point that can be in the form of quantiles, intervals, density functions, and confidence intervals (Hong 2016).

PLF can be used as a stand-alone method. However, it can also be paired with other forms of load forecasting to reduce uncertainty. Additionally, PLF can be used to sample multiple load forecasting methods to produce a stronger estimate of future load. By augmenting multiple load forecasting methods such as regressions, exponential smoothing, or artificial neural networks with PLF sampling, more accurate load forecasts can be built (Xie, Hong, and Stroud 2015).

PLF methodologies often begin by creating a representative day based on a power system, for example, a seasonal representative day that can include weekends, weekdays, and holidays through significant weather changes throughout the year. These representative days can be produced through load forecasts or historical averages. System uncertainties and probabilities, for example, using several decades of weather data, can be used to create a probability density function of variables that affect electricity load. From the original representative day, a new probabilistic spread can be produced (Hong 2016). As countries utilize this method, the first cases should be tested against known data using standard probability metrics (Gneiting and Katzfuss 2014; Zhang, Wang, and Wang 2014; Arora and Taylor 2016). 


\subsection{Periodic Updating and Revisions}

The world is in constant transition, and so are the realities that should be considered in any planning activity. A load forecast is based on an understanding of the load and potential futures at a single point in time. Although the load forecast can be used for planning, its inputs and results may become obsolete before it is even finalized. Therefore, it is important that load forecasts are not conducted as one-time exercises every 5 or more years; rather, they should be updated annually or biennially.

Load forecasting activities are ideally part of a continual cycle of forecasting, assessing, and revising to ensure forecasts are based on the best information available. This aids in ensuring integrated resource planning and other power sector planning activities are based on the most upto-date and accurate forecasts. This updating process allows for ex-post evaluations of forecasts to address the ex-ante unpredictability that input parameters will inevitably have on results, such as significantly decreased economic activity or population growth, (Carvallo et al. 2016). 


\section{Load Factor Forecasting}

A power system's load factor is the ratio between average energy demand over the entire year to peak energy demand during the year. The load factor represents the proportion of the generation fleet that needs to be online on average and demonstrates how efficiently capital is deployed to create generating assets to respond to that peak demand. A low load factor means power plants are sitting idle, which is not economical. A higher load factor indicates that the load profile is smoother, and that infrastructure is better utilized.

$$
\text { Load Factor }(\%)=\frac{\text { Annual Energy }(M W h)}{8760 \text { hours }} \times \frac{1}{\text { Peak Demand }(M W)}
$$

While independent variables affecting demand forecasts were covered in Section 2, load factor is highly dependent on the value and timing of peak load. The primary drivers of peak load are informed by customer sector. For example, residential electricity usage may crest during times of high air conditioning, heating, or television use. For example, in Sri Lanka, electricity planners account for important cricket matches for predicting peaks. The commercial sector peak demand is dependent on business hours. In Pakistan, the ministry of energy mandated a national shift of store and office opening hours at one point to help with blackouts due to a power generation shortfall. In the industrial sector, upticks in aggregate electricity demand are influenced by such factors as:

- Types of industries and amounts of production

- Seasonality of that production

- Single, double, or triple shift workdays

- Auto-generation (also called captive or self-generation) and shifts of aggregate generation equipment installation.

The peak hour of electricity demand comprises the proportionate shares of these customer sectors and may either moderate or exacerbate the value of the peak that planners must meet. The ratio of system peak demand and the percentage contribution from each sector (based on individual sector peak demand) is known as the "coincidence factor" (Bary 1945). Similar to load factors, the coincidence factor helps to quantify how complementary the electrical system is. If all consumer sectors have a high-power utilization at the peak hour of demand, then it means that their demand is coincident, and more could be done to spread this customer demand over the day or year and create a "flatter" electrical load profile. This in turn maximizes generation utilization and minimizes capital stock inefficiency. 


\section{Trends That Affect Load and Load Factor}

Electricity demand can be broken down into many different parts. Examples include industrial, residential, and commercial demand. Electricity demand can also be separated by geography or local climate. In this way, individual trends that affect demand can be examined. If, for example, it is assumed that residential homes become more efficient as countries become more affluent, then load growth may slow. The speed at which it slows can be estimated by different scenarios of the adoption of energy efficiency measures for the residential load, and then integrating the results back into the summed electricity demand. Based on literature, case studies, and interaction with Moroccan officials, below are provided some trends that may affect demand growth in the Moroccan electricity sector that should be examined and potentially modelled in future work.

\subsection{Macro Trends}

Typically, as an economy develops, electricity intensity (MWh/GDP) increases. Economies may shift from an agricultural base to an industrial base. Agricultural and industrial sectors have flat power demand curves and can be met by base-loaded resources. The next economic shift may be from an industrial economy to a service economy. In a service economy, a higher percentage of total electricity demand is from the residential sector with a more distinct daily profile. There is less around-the-clock power demand (i.e., less baseload) and a relative increase in peak demand, which negatively impacts the load factor. However, going forward, major shifts in electricity trends could impact this load factor relationship, and thus impact the forecasted load factor. Some of these trends can benefit load factor and some can exacerbate inefficiency. The net result will depend on which trends prevail and to what extent.

For example, distributed generation such as rooftop solar PV generally implies that net demand for power will decrease. This downward effect in the numerator of the load factor equation has a negative effect, with a decrease in load factor. However, the extent to which distributed generation decreases load factor may change with increased installations of behind-the-meter storage. With storage of solar generation, users will be able to shift their peak demand (e.g., in response to time-of-use pricing assigns different prices to different seasons and hours of the year).

\subsection{Energy Efficiency}

Another trend that will affect load factor is the penetration of energy efficient technologies.

Regardless of a country's policy and mandates, energy efficient technology that increases market share globally may eventually be pervasive in-country, unless less-expensive, inefficient versions exist. For example, in Tunisia, air conditioner installation and use are contributing to a growing peak load and steeper load duration curve. The government both set an efficiency standard and applied a purchase tax on less efficient units to fund the clean energy transition. However, a black market ensued, where electricity customers can purchase unregulated and inefficient units at a fraction of the cost. Barring such bifurcated markets, energy efficient technology generally decreases baseload demand and possibly flattens the load profile shape, having a beneficial impact on load factor. 


\subsection{Industrial Sector Growth}

Patterns of industrial sector growth will influence load factor. As new industries arise and create new economic opportunities, it is important to understand their energy intensity and load fluctuations. The usage pattern, both seasonal and daily, of various industries can also be a key driver. For example, data centers often have a nearly flat energy demand, which has a beneficial increasing effect on load factor. Furthermore, industrial demand response programs aimed at reducing system peak load can dwarf the effects of residential time-of-use pricing programs depending on the proportionate share of each customer sector. Industrial demand response and energy efficiency programs, however, can often only provide tens of hours per year at a very high capacity. As new industries grow, tailored load forecasting for demand, supply, and demand-side management tools as part of a bottom-up load forecast.

\subsection{Electrification and Demand Response}

Referring to end-use devices, increased electrification will impact load factor. In addition to the obvious impact of electrifying the transport sector, the electrification of both water and space heating systems is likely to impact peak load. Furthermore, even for residential customers, there is an increased plug load, despite the fact that appliances are generally more efficient.

A major interest in electrification are electric vehicles. Electric vehicle policies and the speed at which electric vehicles are penetrating the transport sector can largely swing the load factor. At the most basic level, electric vehicles may accentuate the evening peak that already exists, as workers return home and plug in their cars. However, time-of-use programs can shift that usage, especially if there is a prevalence of fleet programs or larger demand customers (Fitzgerald, Nelder, and Newcomb 2016; Kapustin 2020). It remains to be seen how utilities may benefit from electric vehicles, both for increasing revenue, and ultimately possibly using vehicle-to-grid technology for accessing grid services from electric vehicles (Fitzgerald, Nelder, and Newcomb 2016).

As part of widespread electrification, customers can also become active participants of electricity buying and selling. With the increasing prevalence of Internet of Things, they may utilize household automation or blockchain technology to buy and sell their own power, becoming market participants. This will impact the load factor. However, the extent of that is very dependent on how the economic signals get relayed to and through the individual players. It remains to be seen how it will impact the daily and seasonal net load that the utility is tasked with meeting.

Another part of demand response is related to time-of-use pricing. The implementation of timeof-use pricing is dependent on smart metering, whereby a customer's visibility into their electricity usage (both amount and time incurred) coupled with knowledge of the tariff associated with that time period can help shift demand. Ultimately, smart technology can help electricity demand match resources available on the grid. Home automation, such as devices that automatically detect and adjust for electricity prices, will soon become available and can be supported by policy to positively affect the electrical grid, especially for larger energy customers (Neukomm, Nubbe, and Fares 2019). 
In summary, measures can be taken to influence peak load whether seeking to shift the timing or to reduce the overall peak. Measures will work best when used in combinations selected to match system conditions and the local economy (Hale et al. 2016). 


\section{Load Forecasting for the Morocco Power System}

This section discusses short-, medium-, and long-term load forecasting timescales. Each of these timescales provides different information and operational value to energy system decision makers (Hahn, Meyer-Nieberg, and Pickl 2009). Long-term forecasts predict how the system will change over years and decades, and how that system demand can be met by a portfolio of resources some of which may need to be constructed. Medium-term forecasting predicts the next months to years of demand with the goal of planning operations such as near-term impacts of power plant commissioning, decommissioning, and scheduled outages, as well as reserve margins. Short-term planning is concerned with hour to week-ahead forecasts that help to produce daily dispatch.

\subsection{Previous ONEE Load Forecasting}

Following the methodology outlined by the International Atomic Energy Agency, ONEE has conducted an initial long-term load forecast based on socio-economic factors. This methodology uses a scenario-based approach to predict low- and high- growth scenarios, as well as a base scenario. Table 2 shows the assumptions that went into the MAED model ("Modelling for Analysis of Energy Demand (MAED-2)" 2006). Based on these scenarios, ONEE predicted a 2030 annual load of 50.3 terawatt-hours (TWh) in the low-growth scenario, 58.6 TWh in the base scenario, and 69.3 TWh in the high-growth scenario. 
Table 2. Long-Term Load Forecast Scenarios

\begin{tabular}{|c|c|c|c|}
\hline Scenarios & $\begin{array}{l}\text { Determining } \\
\text { Factors }\end{array}$ & Assumptions & Sources/Comments \\
\hline \multirow{2}{*}{$\begin{array}{l}\text { Base } \\
\text { Scenario } \\
\text { (3.9\% } \\
\text { Average } \\
\text { Load } \\
\text { Growth) }\end{array}$} & Economic growth & $\begin{array}{l}\text { - An annual GDP average growth rate } \\
\text { of } 3 \% \text { over the forecasting period. }\end{array}$ & $\begin{array}{l}\text { - Projections provided by the High } \\
\text { Commission for Planning (HCP). } \\
\text { supplemented by those of the World } \\
\text { Bank and International Monetary } \\
\text { Fund. } \\
\text { - } 3 \% \text { also corresponds to the average } \\
\text { GDP growth rate recorded over the } \\
\text { last } 5 \text { years. }\end{array}$ \\
\hline & $\begin{array}{l}\text { Socio- } \\
\text { demographic } \\
\text { development }\end{array}$ & $\begin{array}{l}\text { - The population is expected to } \\
\text { increase from } 35.6 \text { million in } 2019 \text { to } \\
39.3 \text { million in } 2030 \text {. } \\
\text { - The number of households is } \\
\text { expected to increase from } 8.3 \text { million } \\
\text { households in } 2019 \text { to } 10.4 \text { million } \\
\text { households in } 2030 \text {. } \\
\text { - The urbanization rate should } \\
\text { increase from } 62.9 \% \text { in } 2019 \text { to } 67.8 \% \\
\text { in } 2030 \text {. }\end{array}$ & $\begin{array}{l}\text { Projections provided by the HCP } \\
\text { based on the latest general census. }\end{array}$ \\
\hline \multirow{2}{*}{$\begin{array}{l}\text { High- } \\
\text { Growth } \\
\text { Scenario } \\
\text { (5.3\% } \\
\text { Average } \\
\text { Growth) }\end{array}$} & Economic growth & $\begin{array}{l}\text { - A GDP growth rate of } 4 \% \text { over the } \\
\text { forecasting period. }\end{array}$ & $\begin{array}{l}\text { Economic growth slightly above } \\
\text { average. }\end{array}$ \\
\hline & $\begin{array}{l}\text { Socio- } \\
\text { demographic } \\
\text { development }\end{array}$ & $\begin{array}{l}\text { - The same as for the base-case } \\
\text { scenario. }\end{array}$ & $\begin{array}{l}\text { Same as Base Case. Projections } \\
\text { provided by the HCP based on the } \\
\text { latest general census. }\end{array}$ \\
\hline \multirow{2}{*}{$\begin{array}{l}\text { Low- } \\
\text { Growth } \\
\text { Scenario } \\
(2.6 \% \\
\text { Average } \\
\text { Growth) }\end{array}$} & Economic growth & $\begin{array}{l}\text { - A GDP growth rate of } 2.5 \% \text { over the } \\
\text { forecasting period. }\end{array}$ & Below-average economic growth. \\
\hline & $\begin{array}{l}\text { Socio- } \\
\text { demographic } \\
\text { development }\end{array}$ & $\begin{array}{l}\text { - The same as for the base-case } \\
\text { scenario. }\end{array}$ & $\begin{array}{l}\text { Projections provided by the HCP } \\
\text { based on the latest general census. }\end{array}$ \\
\hline
\end{tabular}


Figure 6 and Figure 7 show the growth of peak system load and annual energy load, respectively, as forecasted by ONEE.

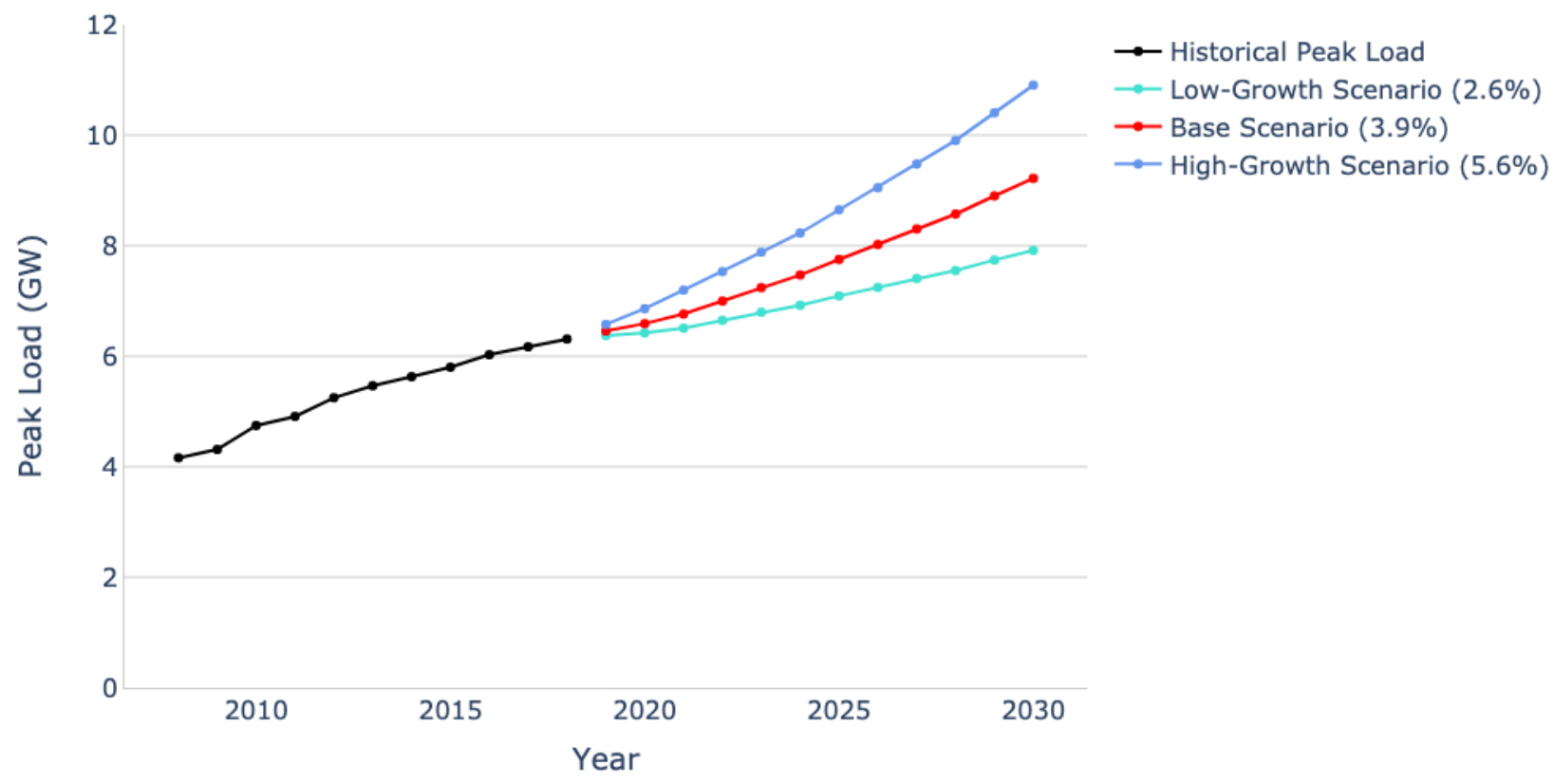

Figure 6. Peak system demand growth as forecasted by ONEE

Source: Data provided by ONEE and figures produced by NREL

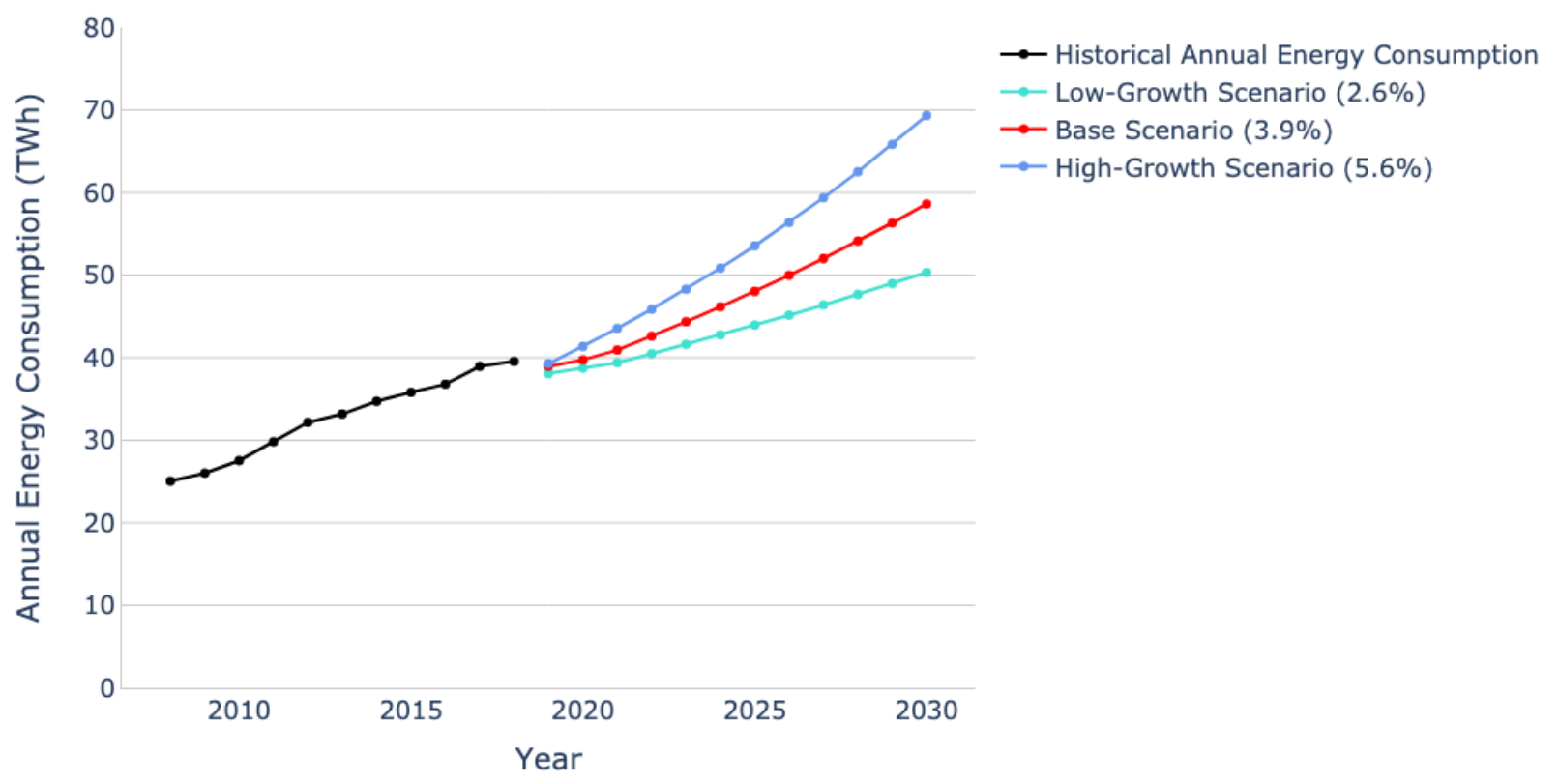

Figure 7. Annual energy demand growth as forecasted by ONEE

Source: Data provided by ONEE and figures produced by NREL 


\subsection{NREL Long-Term Load Forecast for ONEE}

Section 9.1 discussed previous forecasts of load growth from ONEE, which focused on assessing three different scenarios of load demand based on different perspectives of economic growth. Here, these scenarios are expanded by coupling with an important economic indicator, the GDP. Many drivers of GDP are also energy consuming processes. Industries such as manufacturing and service can be energy intensive and can drive energy growth (Moral-Carcedo 2017; Sangrody and Zhou 2017). Additionally, as a country becomes wealthier, it can also increase energy intensity of individuals as technologies such as air conditioning become widespread.

Historical energy consumption and GDP are used to predict future consumption and GDP with two methods: linear regression and logarithmic regression. Linear regression assumes that the relationship between the two factors will remain constant into the future. However, logarithmic regression can also be used as an alternative where GDP growth may continue, but consumption flattens. This flattening may naturally occur as energy intensity for a country flattens with natural deployment of more energy-efficient technologies, as well as through government programs such as energy efficiency measures and new building codes.

Figure 8 illustrates the historical GDP of Morocco through 2019 and projects three levels of GDP growth through 2030, using ONEE's projections described in Section 9.1. Figure 9 illustrates the historical relationship between GDP and both peak load and annual load. With only eleven data points, it is hard to discern a dramatic difference between different regressions relating the GDP to load, but all regressions illustrate, unsurprisingly, that an increase in GDP corresponds to an increase in both annual load and peak demand.

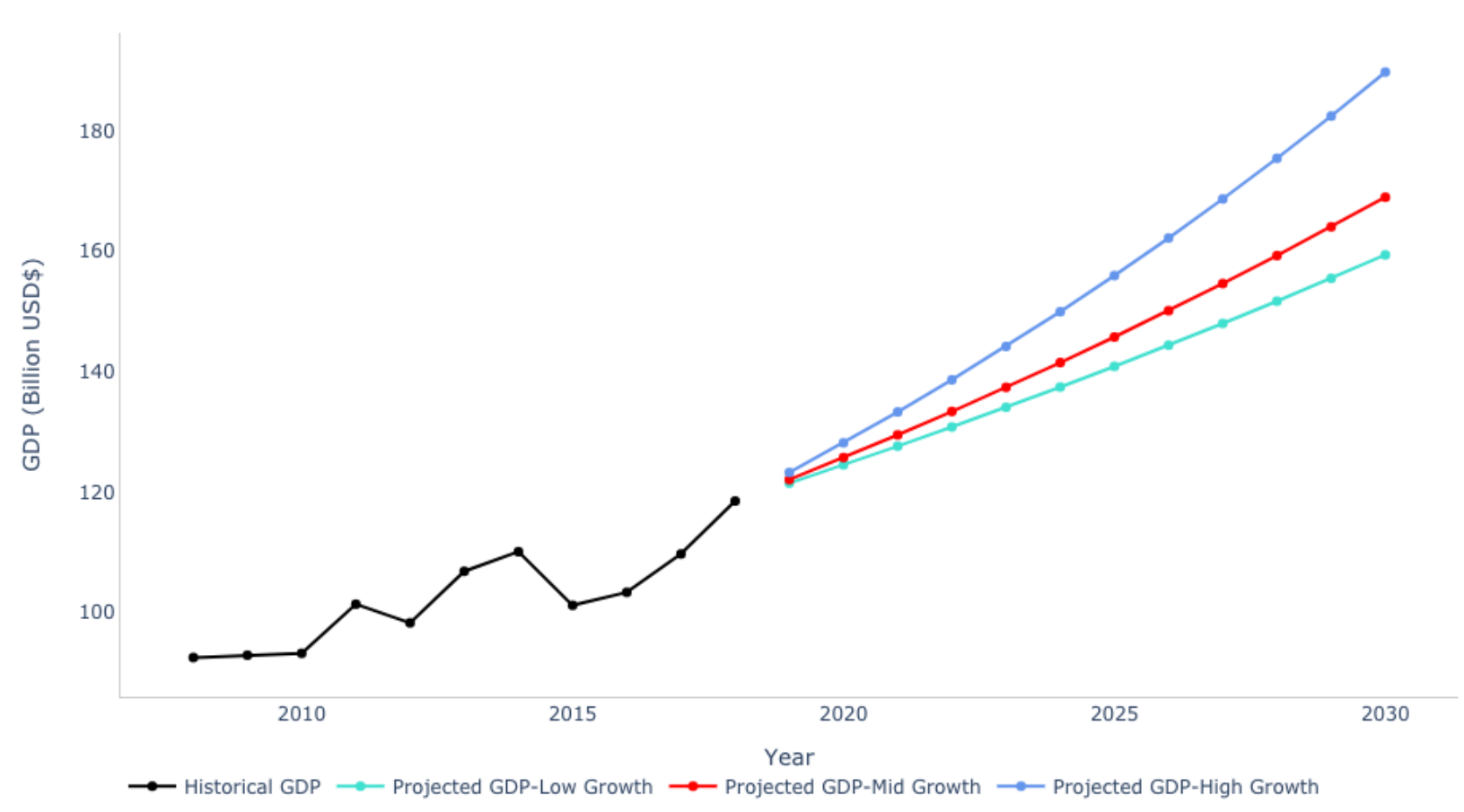

Figure 8. Three projected levels of GDP for Morocco through 2030 


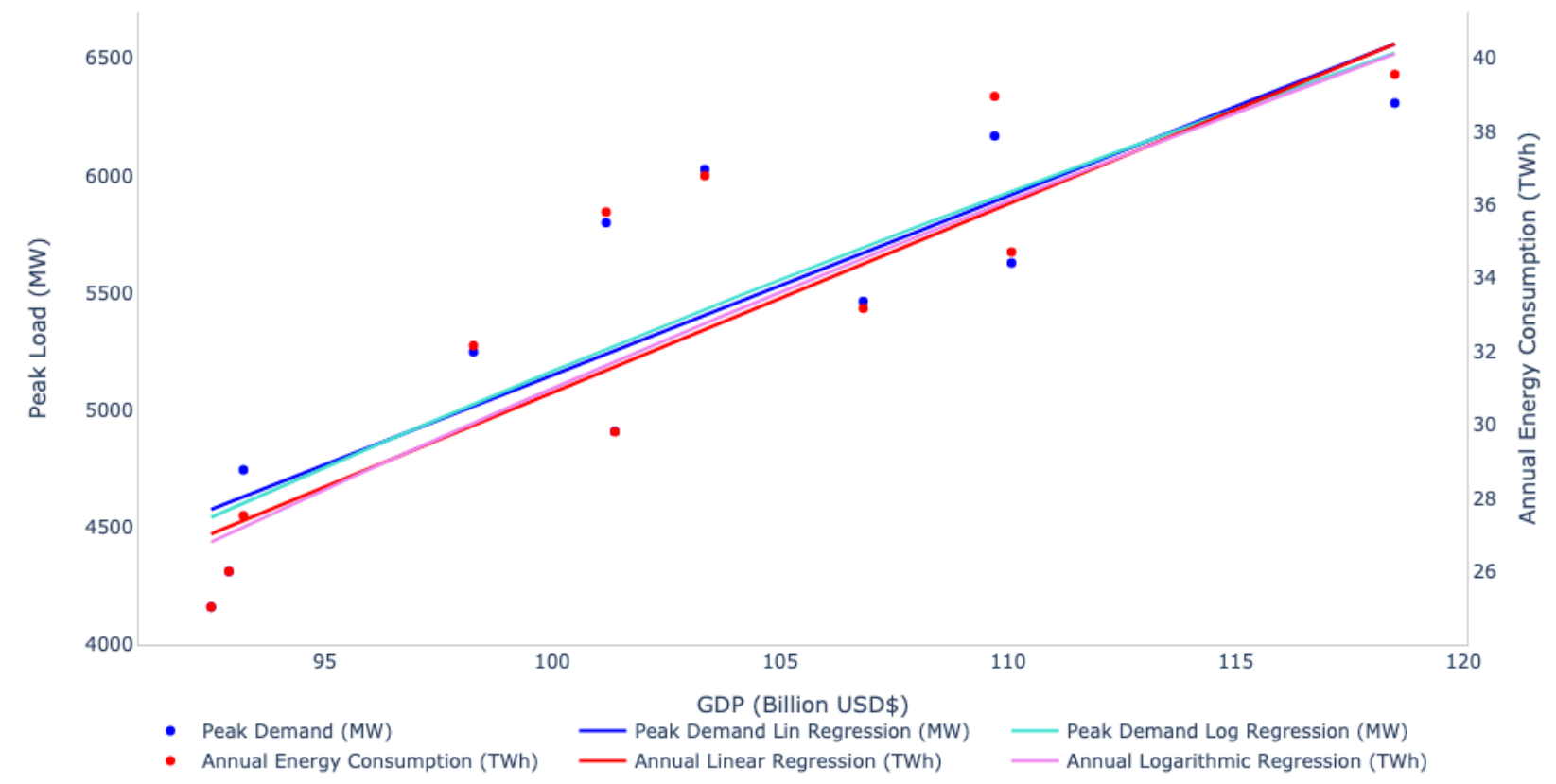

Figure 9. Linear and logarithmic regression of GDP, peak load, and annual energy consumption

Figure 8 indicates that the GDP of Morocco is predicted to be between $\$ 160$ billion and $\$ 180$ billion by the year 2030. Figure 10 shows the logarithmic and linear regression values for peak load under the Low- Medium- and High-GDP growth scenarios. Although the two regressions look similar over historical GDP values, the two regression methods diverge for higher GDPs, with the linear regression predicting a 20\% higher peak in the High-GDP growth scenario.

Figure 11 illustrates a similar trend for the annual consumption regression.

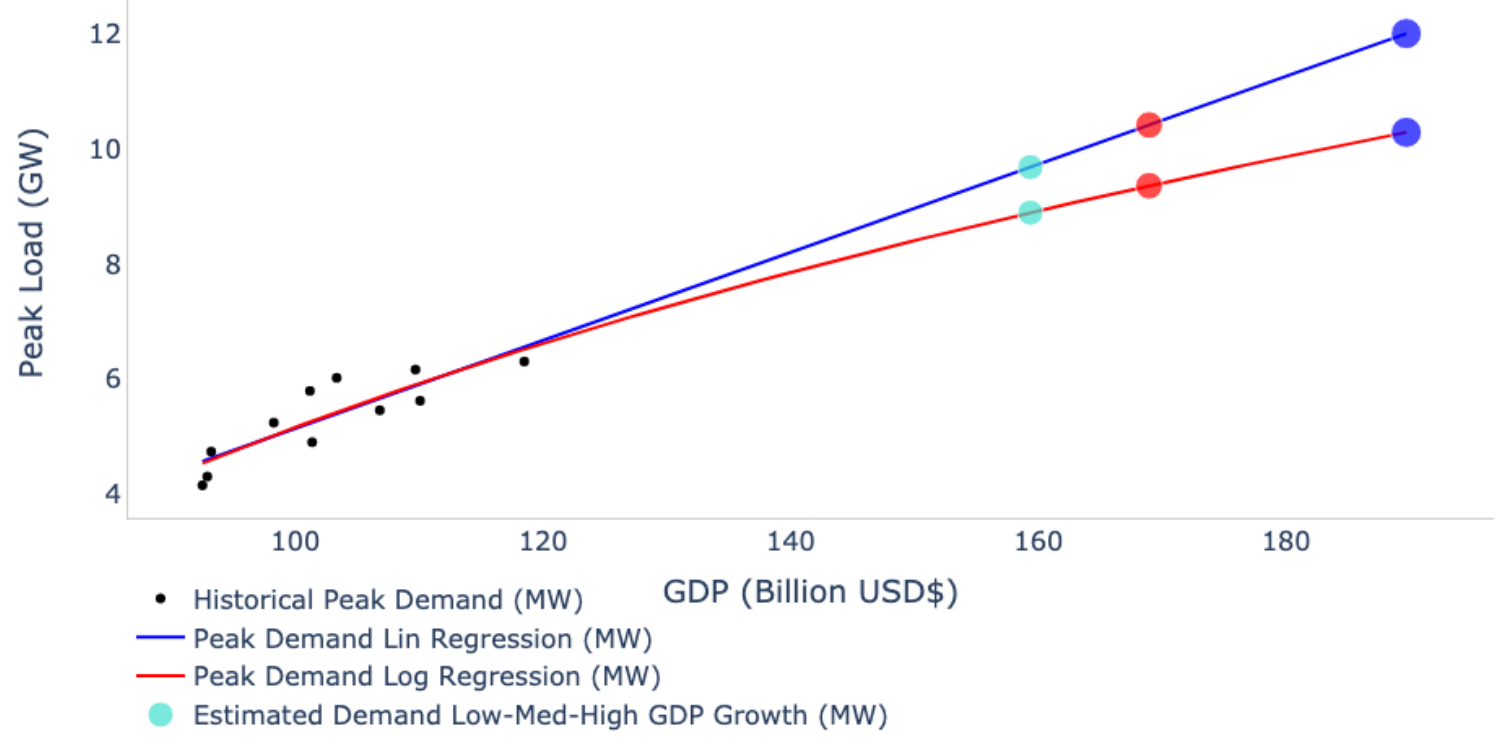

Figure 10. Peak load versus GDP regression for low, medium, and high growth 


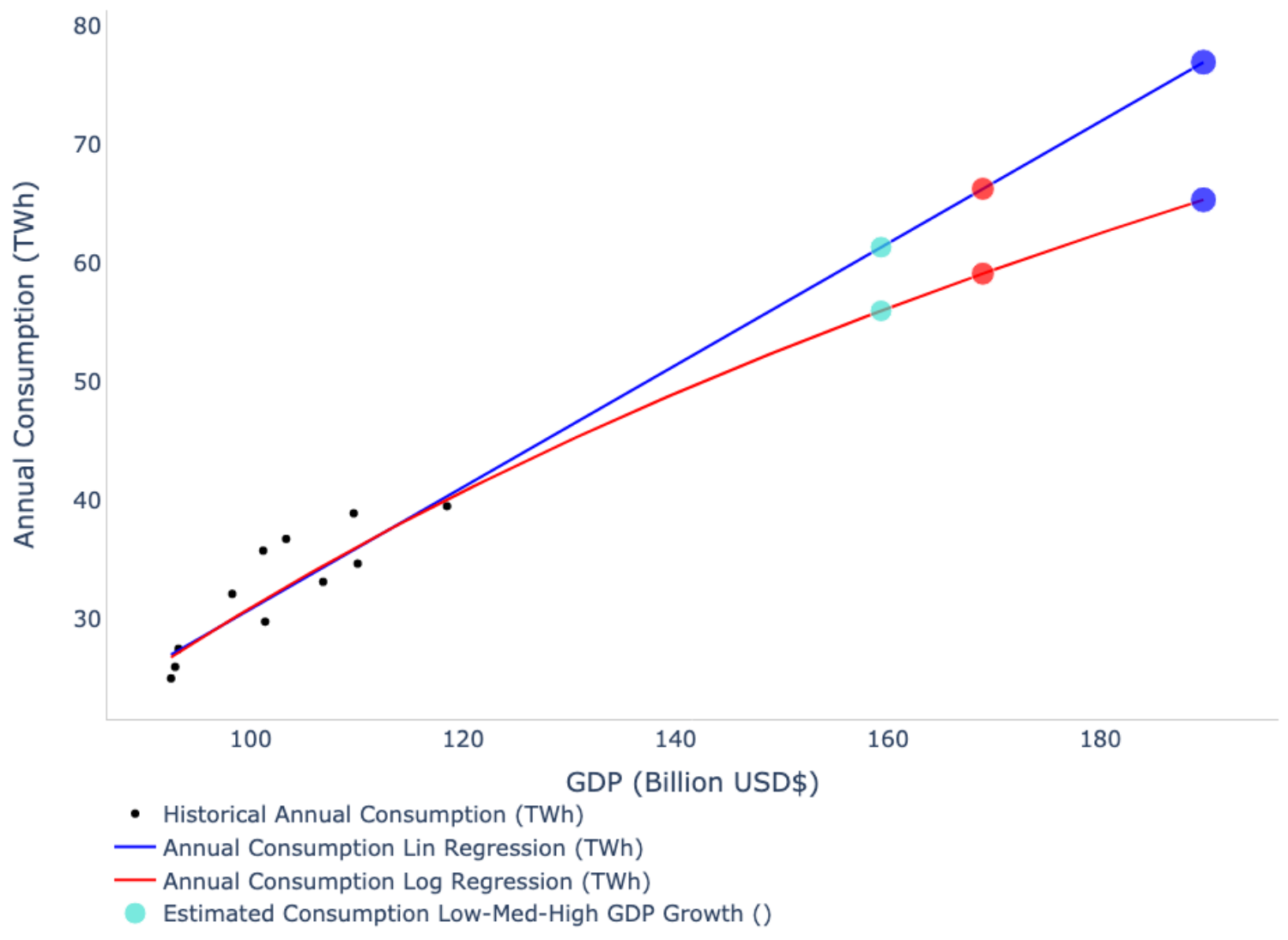

Figure 11. Annual energy consumption versus GDP regression for low, medium, and high growth

Table 3. GDP Base Load Forecast for 2030

\begin{tabular}{|c|c|c|c|c|c|}
\hline $\begin{array}{l}\text { Growth } \\
\text { Scenario }\end{array}$ & $\begin{array}{l}2030 \text { GDP } \\
\text { (Billion USD) }\end{array}$ & $\begin{array}{l}\text { Peak Demand } \\
\text { Linear (MW) }\end{array}$ & $\begin{array}{l}\text { Peak Demand } \\
\text { Log (MW) }\end{array}$ & $\begin{array}{l}\text { Annual } \\
\text { Consumption } \\
\text { Linear (TWh) }\end{array}$ & $\begin{array}{l}\text { Annual } \\
\text { Consumption } \\
\text { Log (TWh) }\end{array}$ \\
\hline Low (2.5\%) & 159.4 & $9,685.0$ & $8,894.3$ & 61.38 & 56.02 \\
\hline Medium (3\%) & 168.9 & $10,417.2$ & $9,361.5$ & 66.31 & 59.16 \\
\hline High (4\%) & 189.7 & $12,004.1$ & $10,289.1$ & 76.98 & 65.38 \\
\hline
\end{tabular}

Understanding that energy intensity (kWh/\$GDP) can be nonlinear, this GDP-based regression can help assess how changing lifestyles can affect electric load growth nonlinearly as Morocco's economy and per capita GDP grow.

The reason for the logarithmic cases is to suggest the value of energy efficiency measures. This logarithmic trend can occur through energy efficiency measures, where the economy can "decouple" economic growth from energy consumption. For example, in the mid-case scenario, the peak system demand difference between the linear and logarithmic projections is 1,056 MW. While it requires significant additional analysis to suggest the time this peak may occur, energy efficiency measures could prevent building this additional system capacity (Weron 2006). This helps to suggest the overall value of energy efficiency measures (Hahn, Meyer-Nieberg, and Pickl 2009; Sangrody and Zhou 2017). 


\subsubsection{Normalized Annual Curves}

After computing the peak and annual energy consumption, the goal of demand forecasting is to better understand how to meet future system needs for planning asset procurement. A prediction is built of the annual load curve on the monthly and hourly basis using simple normalization techniques, understanding that this methodology is simplified but provides a basis for more indepth analysis (Hong, Wilson, and Xie 2014). Importantly, there is a major difference in load curves between weekend and weekday load shapes, but this is often ignored in long-term load forecasting and is more explicitly examined in medium-term load forecasting.

For this section, historical load shapes for Morocco are analyzed and then the same load shape is assumed to persist in the future even under higher consumption levels. Although this approach neglects significant changes that energy demand may face in the future, it is still useful for providing a basis for more a more detailed, bottom-up approach. Figure 12 shows the hourly load for the Morocco system as a 3D surface plot. The plot indicates that daily peak load occurs in the evening, regardless of season. A secondary peak also occurs at midday. July and August show the highest overall load, with lowest load being in December and January. 

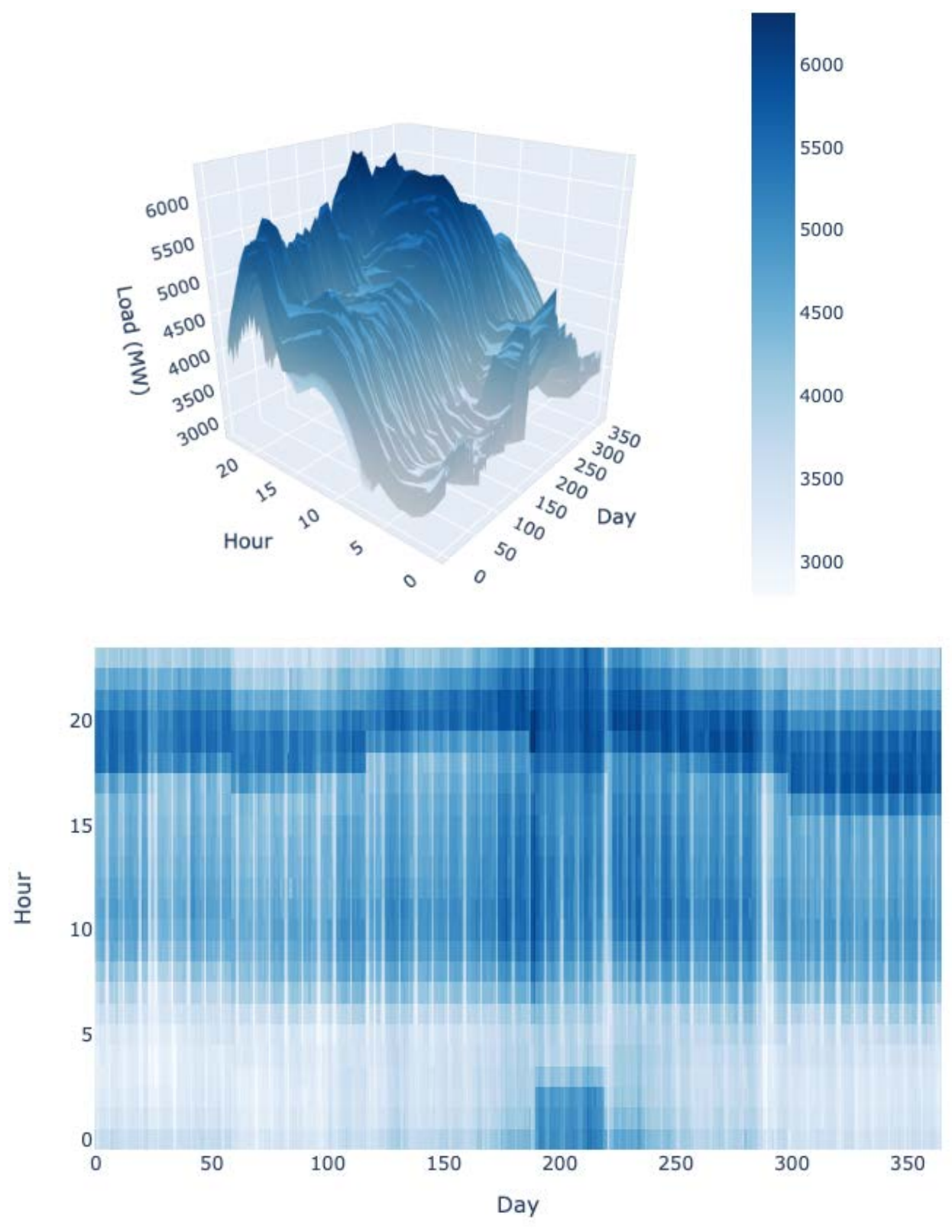

Figure 12. 2018 hourly load data for Morocco

A normalized load curve was tested and averaged for 2008-2017 by creating a projected hourly curve for 2018. By multiplying the normalized curve by the peak system demand for $2018(6,310$ MW) with the projected curve shown in red and the actual curve shown in blue. The average error for this projection is $6.60 \%$ and the standard deviation of error is $5.04 \%$, but the maximum local error is $47.8 \%$ meaning the estimate misses key hours but is a reasonable starting point. Extending this method to 2030 using the low, medium, and high GDP predictions by ONEE, three new curve shapes can be built, as shown in Figure 13.

$$
L(2030, t)=L_{\text {avg }}(t) \cdot P(2030)
$$

where the predicted $P(2030)$ has a low, medium, and high value in conjunction with the predicted linear and logarithmic growths based on GDP, as shown in Figure 13. 


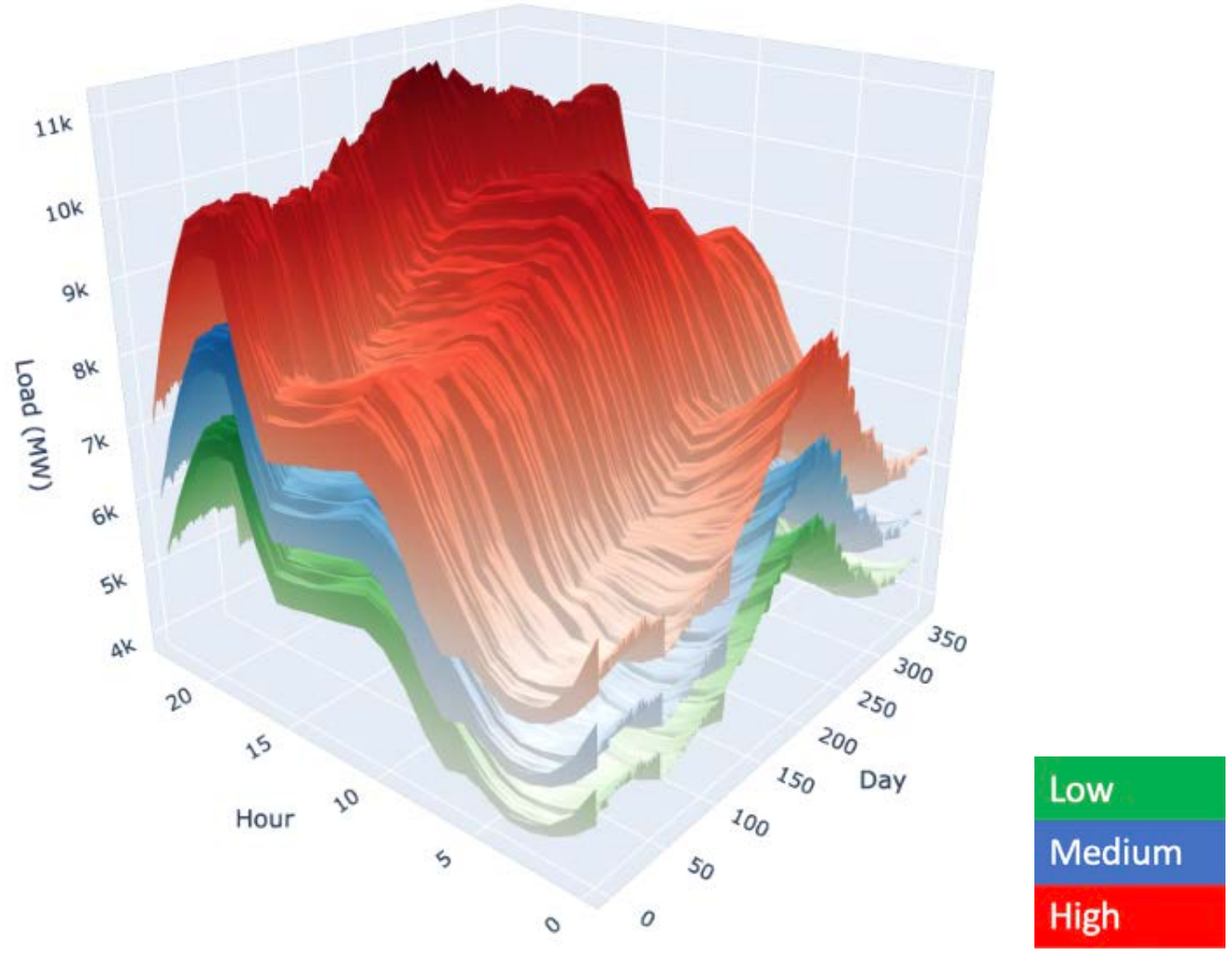

Figure 13. 2018-2030 Low (Green), Medium (Blue), and High (Red) hourly load curves derived from normalized and averaged 2008-2018 data

As discussed above, full hourly annual normalization contains high errors at many data points. Understanding seasonal effects on electricity load can also be important and is essential for proper planning and operation of the power system (Hong, Wilson, and Xie 2014). Monthly averages are examined, and peak curves normalized in a similar fashion for a more focused period of time. Previously, ONEE has conducted analysis that looks at peak demand days in winter, spring, summer, and autumn. This analysis is furthered by examining average and peak demand days by month.

The monthly and peak electricity load are defined as $L_{\text {monthly }}(m, h)$ and $P_{\text {monthly }}(m, h)$, respectively. Here $m$ represents the month and $h$ represents the hour in a day.

$$
\begin{gathered}
L_{\text {monthly }}(m, h) \\
\text { where } m=[\text { Jan, Feb, Mar, ...Dec }] \text { and } h \in \mathbb{Z} \text { where } 0 \leq h \leq 23 \\
P_{\text {monthly }}(m, h)
\end{gathered}
$$

The "average monthly load curve" is calculated by averaging the same hour (1 through 24) across every day in the month. The "peak load day" is the load curve for the day with the highest demand for any single hour in the month. The averages of each month can be taken across years or within a year, depending on the known factors affecting load each year. For example, yearly average temperatures can significantly vary across years and can impact which year should be 
chosen as a representative. 2018 was used as a representative load year and normalized by month, as shown in Figure 14.
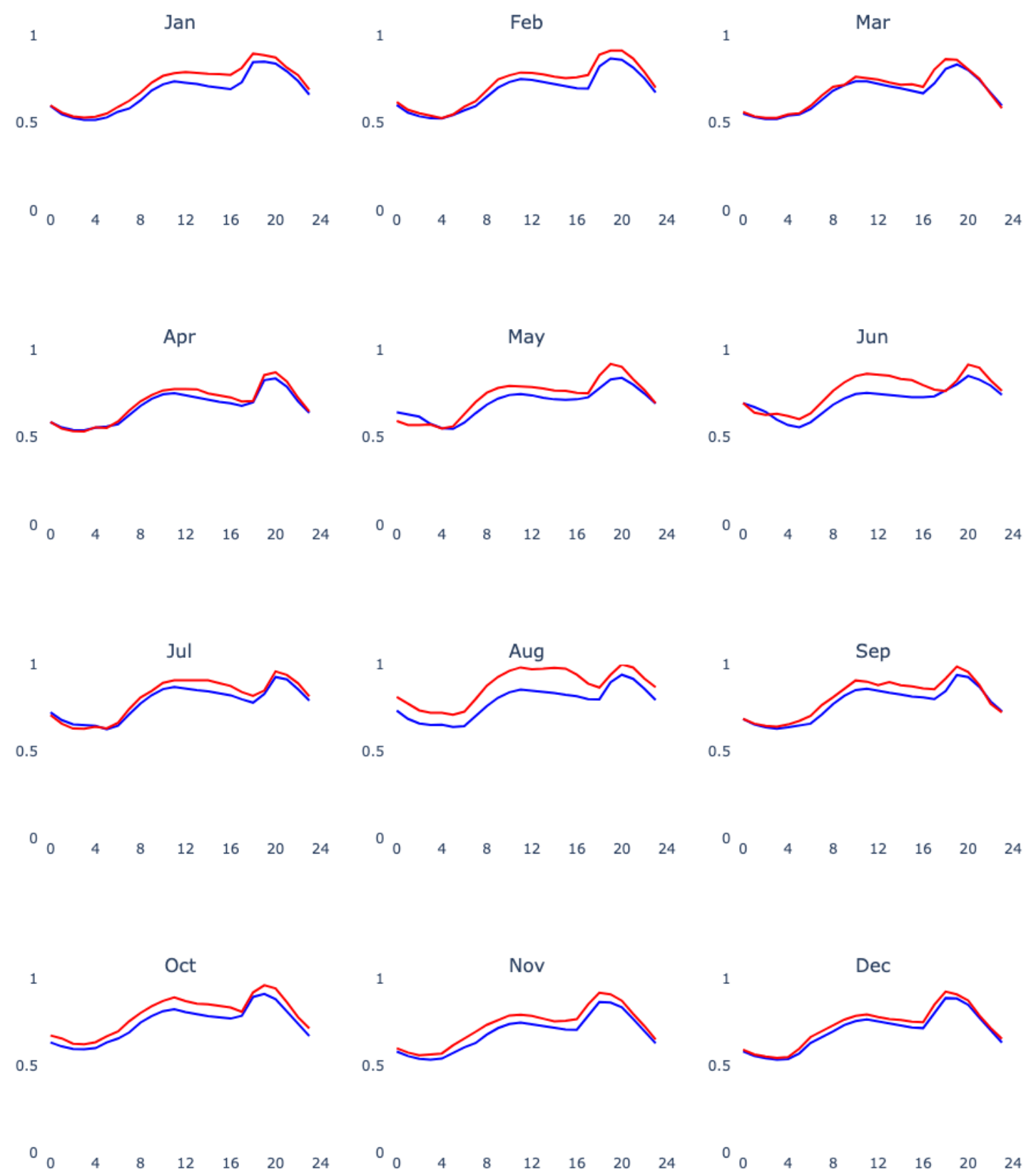

_ Average Monthly Load Curve — Peak Load Day

Figure 14. 2018 normalized monthly load curves for Morocco

These normalized load curves are then multiplied by the peak system load based on the 2030 medium GDP growth scenario to produce an initial estimate for 2030 monthly average load and 
peak load, as shown in Figure 15. This estimate is crude but provides a first step in estimating 2030 monthly loads.
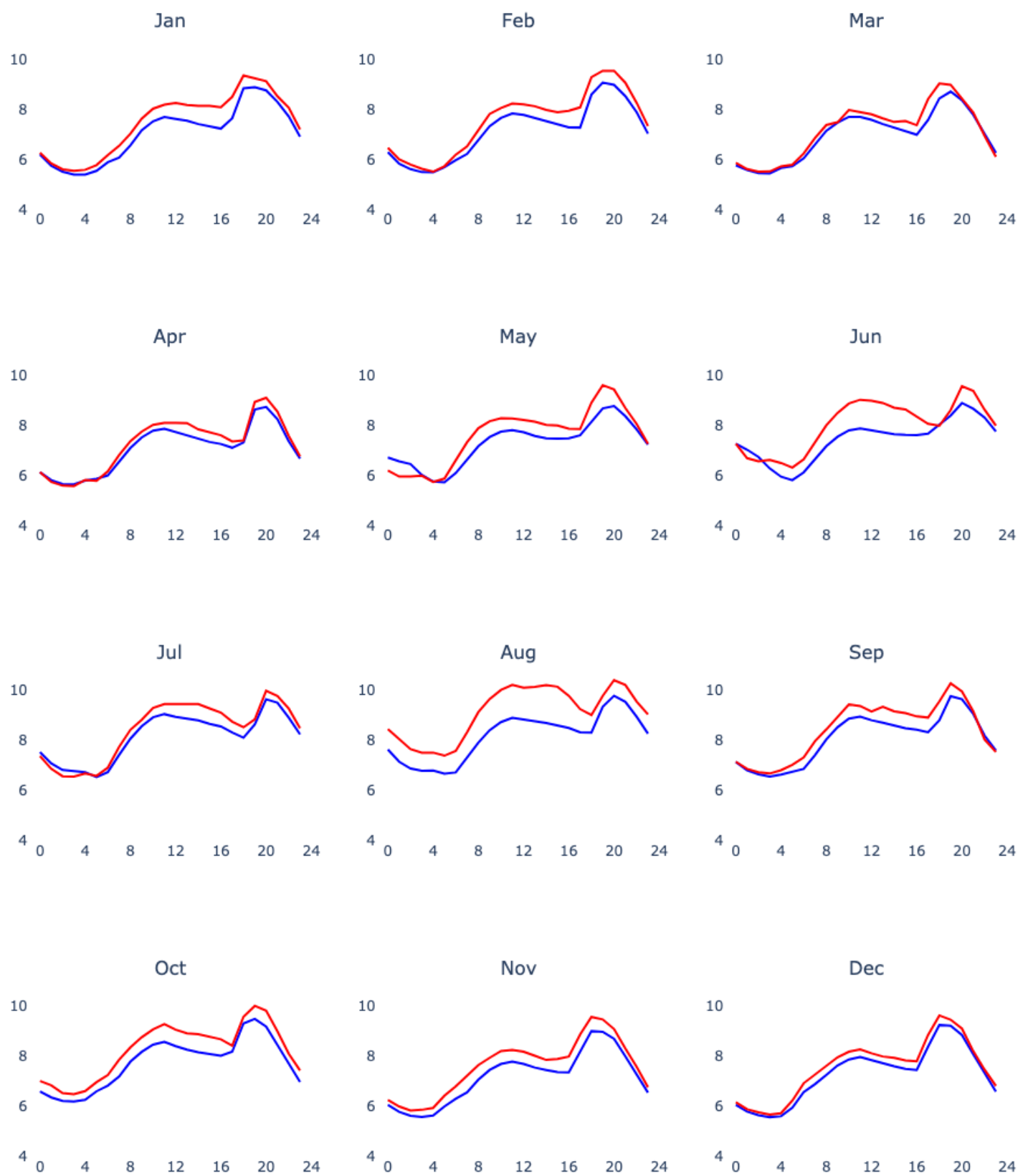

— Average Monthly Load Curve (GW) — Peak Load Day (GW)

Figure 15. 2030 projected monthly load curve 
These curves give a glimpse into the current Moroccan power system and how it may evolve into the future. Firstly, they indicate how "peaky" the system may be, which underscores the importance of flattening the curve to preserve load factor efficiency to minimize an over-build or under-build of capacity in the future; however, it is important to emphasize that the load curve will likely not remain the same shape in the future, given the rapidly industrializing nature of Morocco. Many economic effects could change the shape, such as mass adoption of air conditioning or deployment of electric vehicles. While the above scenarios would drastically reshape the load curve, it also shows that government programs, such as time-of-use rates or energy efficiency programs, can have a significant impact on the load shape and are very important to consider.

\subsubsection{Impact of Solar Energy on Net Load Curve}

Using estimated solar output and forecasted load curves, the impact of different future solar energy capacity penetration levels can be estimated. Morocco, which has a good solar resource given its location, has multiple options for solar energy deployment (Kousksou et al. 2015). In particular, solar PV and concentrating solar power are two solar technologies already being deployed in the country. In this section, the impact that significant PV deployment in Morocco may have on the shape of load is analyzed. Global Solar Atlas data was used from the World Bank to create monthly average solar load curves ("Global Solar Atlas" 2020). ${ }^{3}$ Using these monthly average solar predictions developed, along with the 2030 mid-load case, a range of $\mathrm{GW}$ for potential PV deployment in Morocco is analyzed (1, 3, 5, and $7 \mathrm{GW})$, shown in Figure 16. With these predicted ranges, the net load is calculated, or demand minus contribution from PV in this case (Figure 17), percentage PV penetration, and monthly maximum ramping rate over an hour. Morocco has a goal of 52\% renewable energy by 2030; therefore, the percentage of energy that comes from solar PV and its effect on system behavior is an important consideration.

Figure 16 and Figure 17 indicate how solar from PV can dramatically change the shape of net load, which has been well-documented in other areas of the world (Denholm et al.). In particular, solar PV generation creates a new low net load level in the middle of the day, which might require backing down other sources of generation as well as dramatic evening ramp-up when the PV generation decreases, and the native load continues to increase beyond the daylight hours. These net load shapes may present new challenges for the operation of the Moroccan power grid and should continue to be evaluated as Morocco moves forward with its renewable energy generation goals. These curves speak to the need to do detailed analysis of potential wind and solar resources in the country through long-term monitoring and data weather history to produce a more accurate view of the impact of VRE expansion on the Morocco electricity system.

\footnotetext{
${ }^{3}$ Other sources of PV resource data exist as well. PVWatts is a tool developed at NREL that uses international historical weather data to predict solar direct normal irradiance output and a linear correlation based on installed capacity to estimate PV output ("PVWatts Calculator" 2021). An analysis of PVWatts data shows much more variation in generation than just monthly averages, which makes it a valuable tool for understanding key weather days, such as partly cloudy days or days with very low solar output.
} 

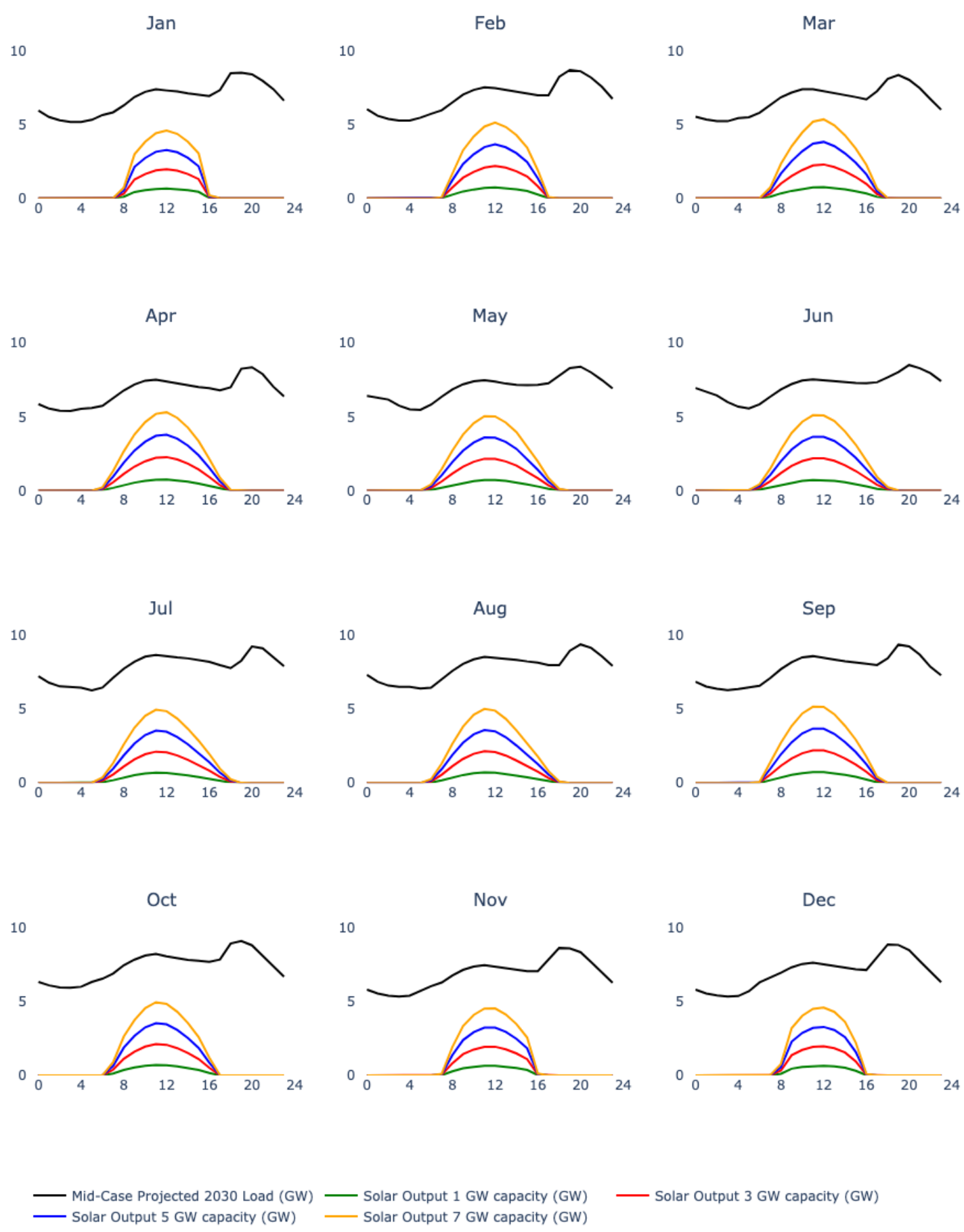

— Solar Output 3 GW capacity (GW)

Figure 16. Monthly average forecast load curves and projected solar output based on installed capacity 

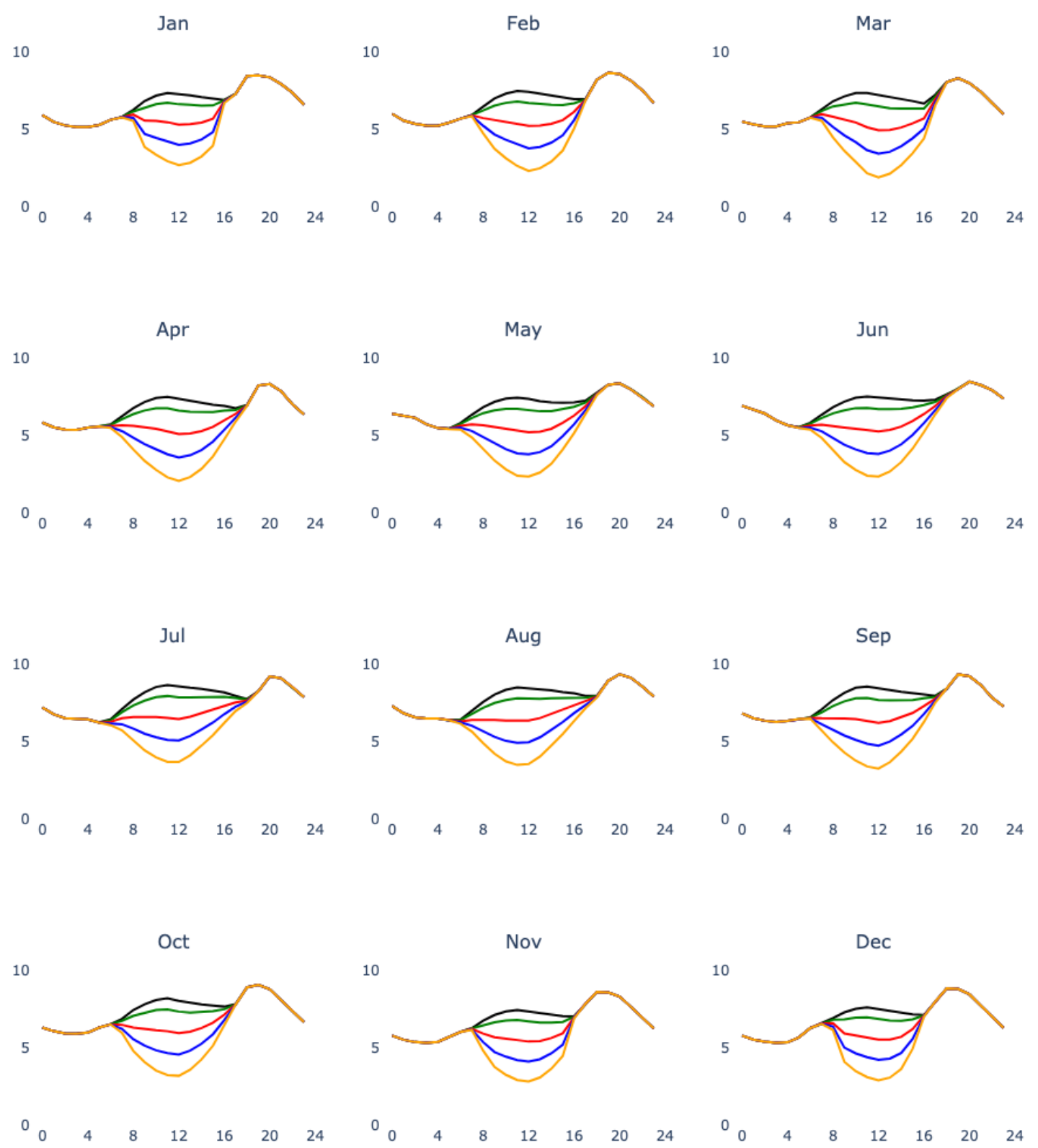

$\begin{array}{ll}\text { Mid-Case Projected } 2030 \text { Load (GW) } & \text { Net Load at } 1 \text { GW Solar PV capacity (GW) } \\ \text { Net Load at } 3 \text { GW Solar PV capacity (GW) } & \text { Net Load at } 5 \text { GW Solar PV capacity (GW) } \\ \text { Net Load at } 7 \text { GW Solar PV capacity (GW) }\end{array}$

Figure 17. Monthly average forecast load curves and forecast net load based on installed solar capacity

\subsection{NREL Medium-Term Load Forecast for ONEE}

Medium-term load forecasting generally seeks to predict electrical load peaks and behaviors for the next 12 months. Based on these predictions plant closures, maintenance, and commissioning schedules can be decided. Additionally, decisions regarding seasonal reserves and new 
procurement can be informed. At the time of writing, only load data from 2008 to 2018 were available. Currently, ONEE does not perform medium-term load forecasting with an annual time horizon. The purpose of this section is therefore to provide example medium-term load forecasts that can be implemented and advanced by ONEE. To demonstrate the accuracy of these methods 2008-2017 data were used and tested against 2018 load data to estimate an approximate error. These methods can then be updated with 2019 data to forecast 2020 load and be updated regularly after that. Two methods were employed for this medium-term forecasting: hourly regressions and load clustering.

\subsubsection{Hourly Regression}

In the long-term forecasting, the normalized load is assumed and multiplied by the projected system peak was a reasonable method to produce the 8760-load curve for the year 2030. While a simple starting method, this method assumes that all hours of the year grow linearly with system peak even in the logarithmic load growth scenario. It is important to note that this simple starting methodology is inadequate for sophisticated load forecasting. Two key variables include ambient temperature and weekday/weekend dates. This can be implemented by adjusting for the first weekend in January across all years or creating seasonal representative weekdays and weekends. Other key variables could also be layered on top of ambient temperature and weekday/weekend variables. These enhancements should be viewed as important next steps in medium-term load forecasting.

Beginning with the simplified methodology, Figure 18 takes a sample day of the year and shows plots of all 24 hours across years. 


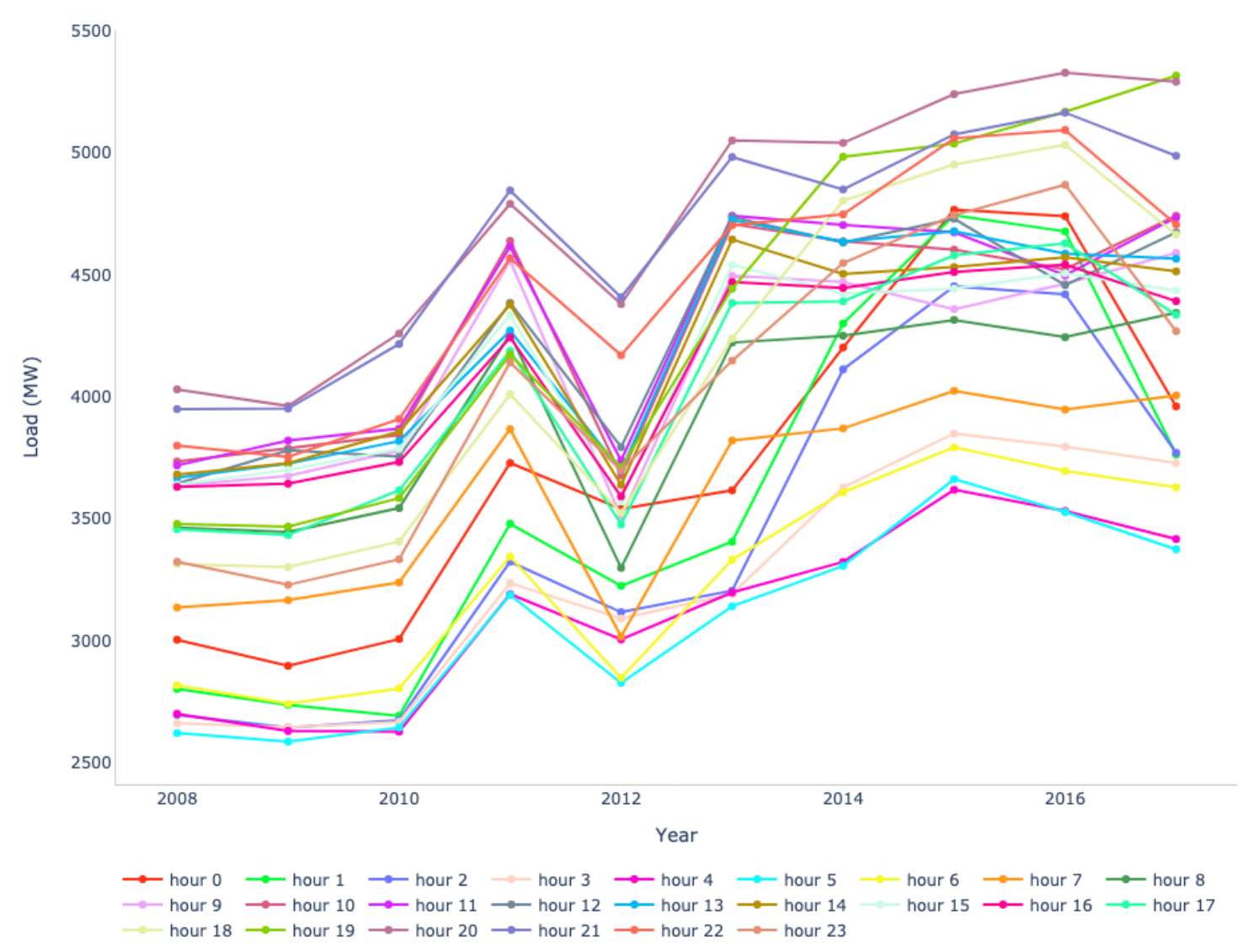

Figure 18. June 124 -hour data across available years

An alternative method is to use linear and multivariable regression on each hour across years, allowing each hour of the year to grow at its own rate (Abu-Shikhah, Elkarmi, and Aloquili 2011). There are multiple regression shapes that could be selected. Linear regression is of course wrong, but also reduces the variance that can come from higher-order polynomials. Another source of error for this method is that important data, such as weekends, become lost, as no awareness is trained into the algorithm between years. Although there are several sources of error, the first attempt at medium-term forecasting was done using a first, second, and third order polynomial regression for 2008-2017 data using the forms:

$$
\begin{aligned}
\text { Linear (first order): } \quad L(2018, t)=a(t) \cdot y+b(t) \\
\text { Second order: } \quad L(2018, t)=a(t) \cdot y^{2}+b(t) \cdot y+c(t) \\
\text { Third order: } \quad L(2018, t)=a(t) \cdot y^{3}+b(t) \cdot y^{2}+c(t) \cdot y+d(t)
\end{aligned}
$$




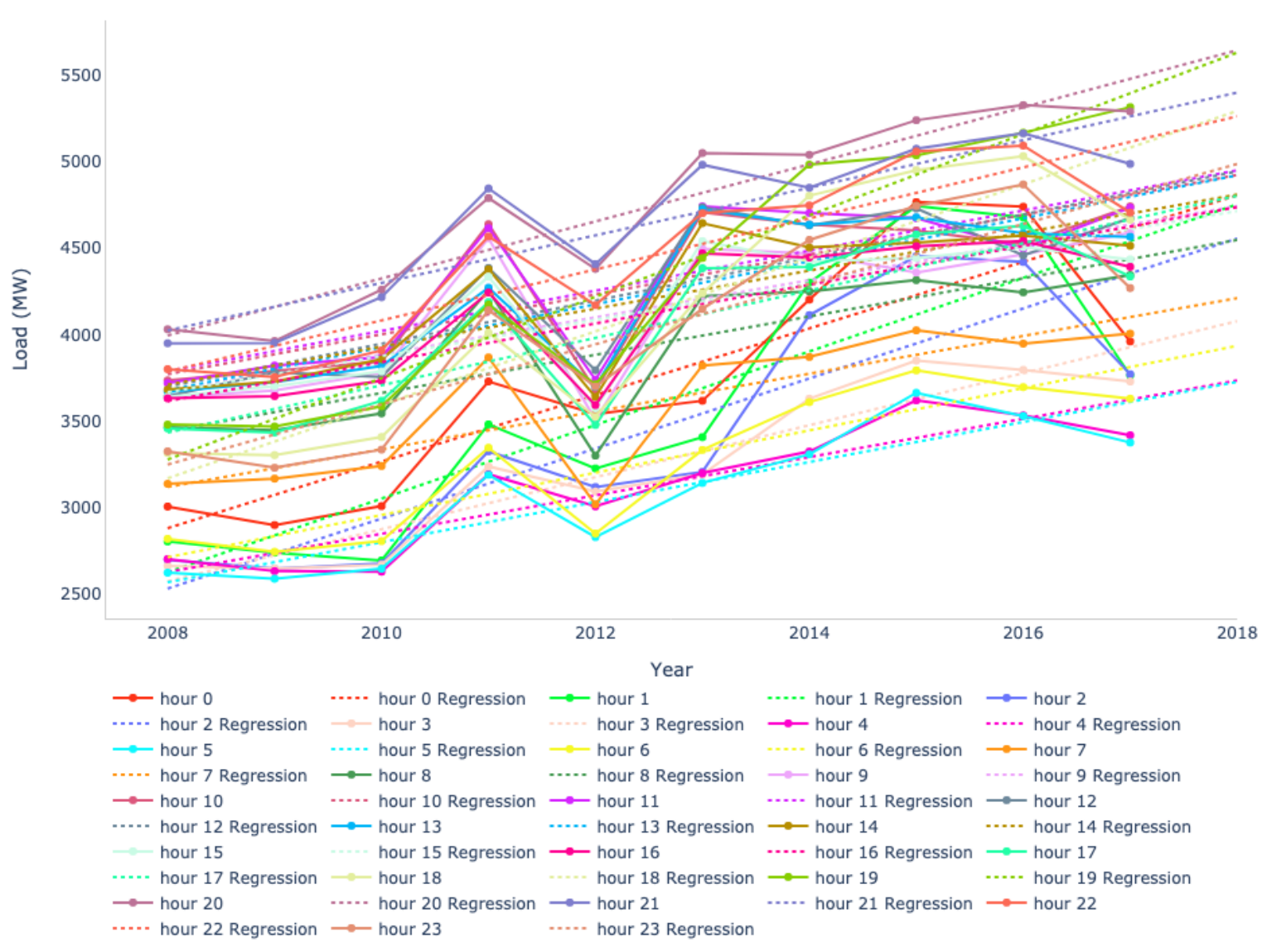

Figure 19. June 124 -hour data across available years with linear regression

In line with the simplified nature of this brief analysis, the regressions shown above does not include in its equations either ambient temperature or weekday/weekend bifurcation. Next steps to this work would be to include increased multivariable regressions.

In Figure 19, each regression has a unique slope. These slopes can very often be very similar but can also vary wildly. By using individual hour regressions, it gives the freedom for key and peak hours to scale differently across years. The average and maximum errors were compared across the three polynomial interpolations and to the long-term load forecasting. 
Table 4. Regression Errors

\begin{tabular}{|l|l|l|l|}
\hline Method & $\begin{array}{l}\text { Average } \\
\text { Error }\end{array}$ & $\begin{array}{l}\text { Error Standard Deviation } \\
\text { (over 8760 samples) }\end{array}$ & $\begin{array}{l}\text { Maximum } \\
\text { Error }\end{array}$ \\
\hline Linear regression & $6.22 \%$ & $6.42 \%$ & $60.6 \%$ \\
\hline $2^{\text {nd }}$ order polynomial regression & $7.84 \%$ & $7.48 \%$ & $65.7 \%$ \\
\hline $3^{\text {rd }}$ order polynomial regression & $12.2 \%$ & $10.3 \%$ & $89.4 \%$ \\
\hline Normalized load curve (Section 9.2.1) & $6.60 \%$ & $5.04 \%$ & $47.8 \%$ \\
\hline
\end{tabular}

Table 4. shows that the regression analysis offers little improvement over normalizing and scaling the load curve as done in Section 9.2.1. However, as was cited in the literature, other regression shapes, such as exponential or logarithmic, could be used. Additionally, important variables, such as weekends, were not considered in this regression and should be examined for this method to be more accurate. Sometimes the wrong polynomial shape can drastically increase the variance and error if regressed over too short of a time frame and extrapolated to long-term data. This method, while not a significant improvement for now, could become more valuable and accurate with additional analysis.

\subsubsection{Load Clustering and Load Duration Curves}

Understanding the relationship between peak and average demand is important to understanding generation assets and energy efficiency programs that can be implemented. A method for this is to establish load duration curves that sort the 8760 hourly demand in a descending order, as shown in Figure 20 (Scott et al. 2019). The purpose of this clustering is twofold. First, to produce a graph showing the flatness of the load duration curve. In an optimized system, this curve would be relatively flat so that the peak-to-average ratio is low, showing an efficient allocation of generation assets and a good optimization of generation asset capacity. Second, this curve can be normalized to the annual peak load to show whether the "flatness" of the curve is increasing or decreasing. As can be seen in Figure 21 where the load duration curves are normalized across years, the relative flatness of the curve is not changing significantly, meaning there may be significant opportunities to match generation and demand to further increase the efficiency of the system. 


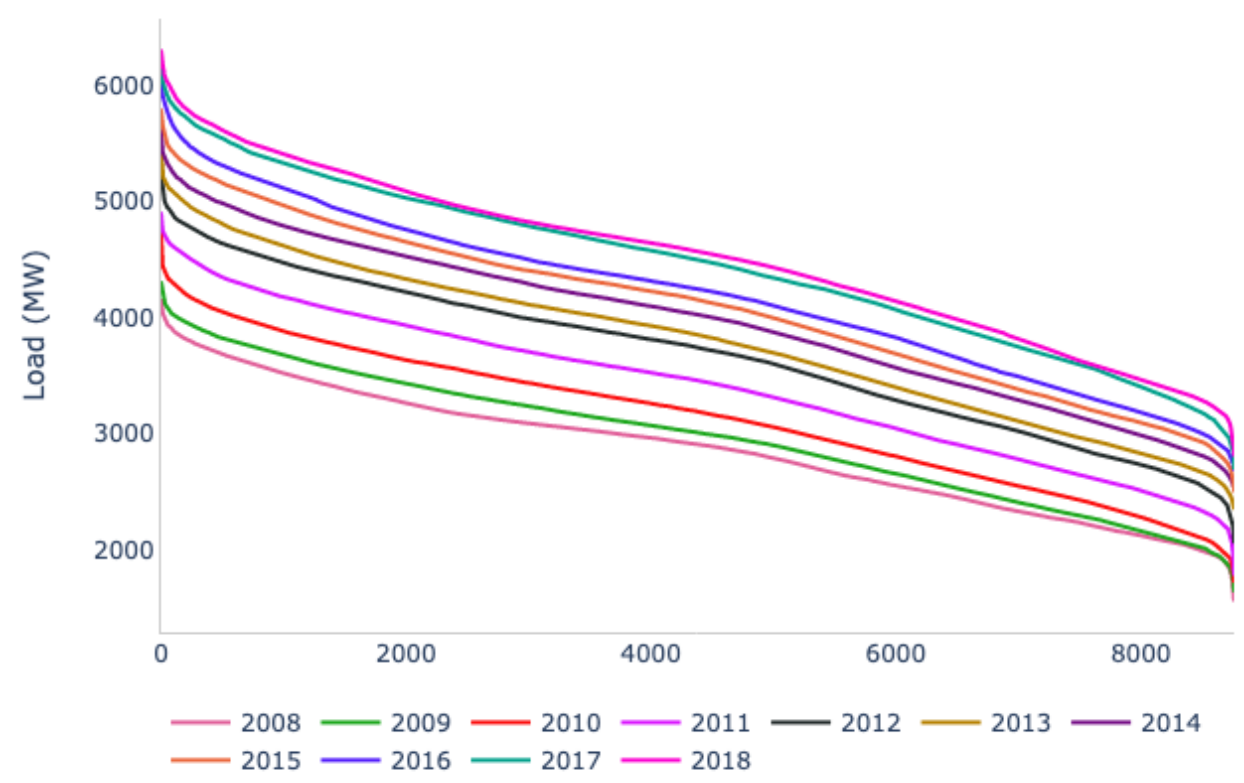

Figure 20. 8760 Load duration curve

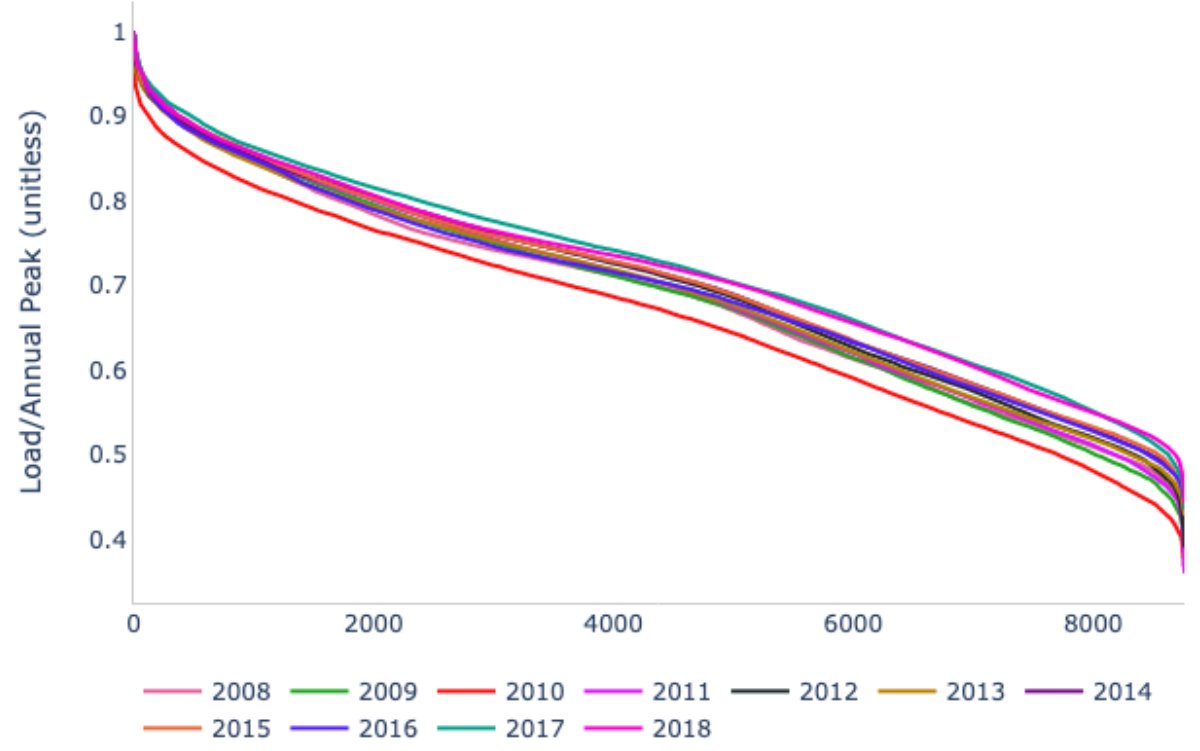

Figure 21. Normalized load duration curves across years

Load duration curves are especially useful in comparison to other electricity systems. In Figure 22, the Moroccan energy system is compared to the Texas system to compare the efficiency and ratio of peak to average load. Texas, being its own synchronous grid at similar latitude as Morocco and having similar solar resources, is a useful comparison point, albeit with a somewhat different load mix (near-universal air conditioning and significant commercial and industrial demand, in addition to residential). Figure 22 illustrates the normalized load duration curve for 11 years in Morocco and one year for Texas. 


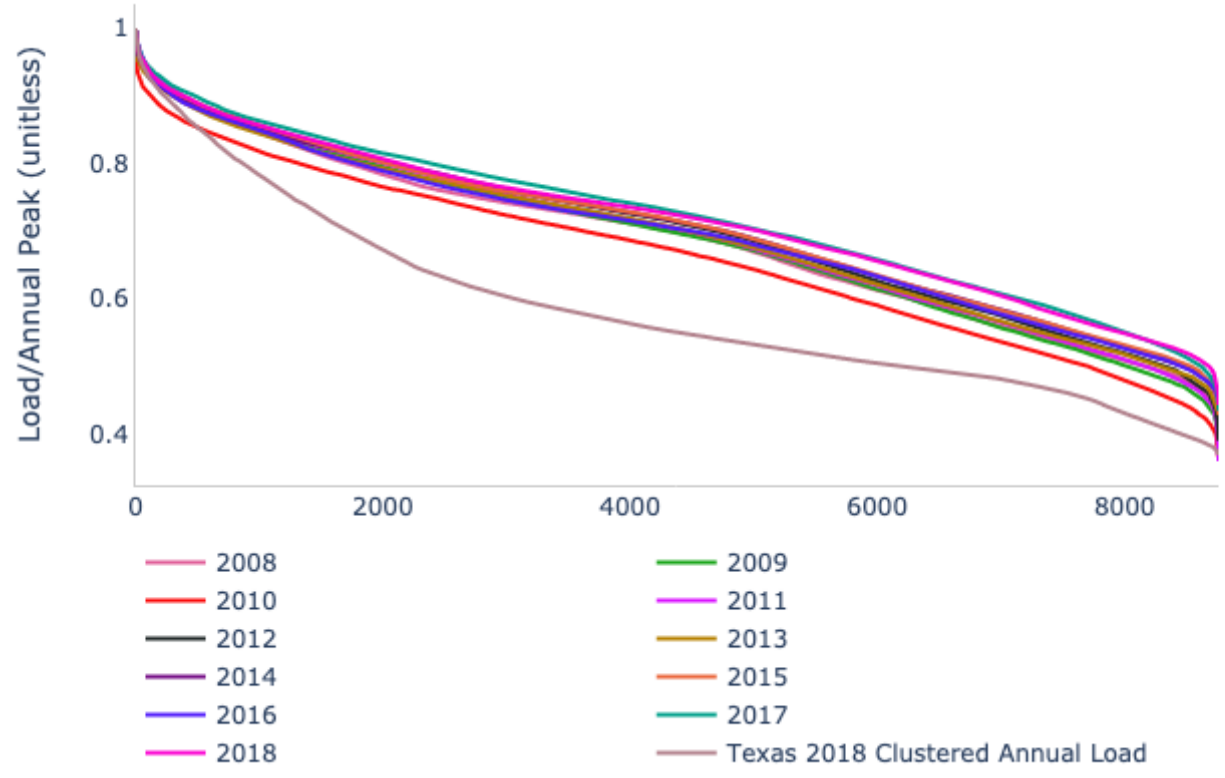

Figure 22. Normalized load duration curves across years with Texas comparison

The Texas curve, shown in Figure 22, is useful for several reasons. For approximately 6,000 hours per year, the Texas electricity system has a mostly flat curve. Meaning during this time, the structure of the Texas electricity system and market can provide insights into efficiently managing their power system. However, for the highest 2,000 hours, the Texas electricity load becomes quite "peaky" and could do more to significantly level load. The Moroccan power system shows no such major change and demonstrates during the peak hours of the year gradual increase. For load duration curves, it is important to emphasize that these hours are not contiguous. Next steps for this analysis include examining the peak 3-hour average ramp-rate. Using Texas as an example for this work could help Morocco compare its peak and examine what mitigations to rapid ramping are successful and unsuccessful in other jurisdictions. Some of techniques could be considered and adapted for the Morocco power system.

A metric commonly used in medium-term planning with load clustering is ratios of the maximum to minimum demand. This can be done on the daily, seasonal, or annual basis (Scott et al. 2019). Table 5 shows the ratio for each year. Although the slope of the normalized curve shown in Figure 22 does not show a significant decrease, the ratio of peak to minimum power is steadily decreasing in the Morocco energy system, which demonstrates progress in terms of resource and system optimization. System optimization such as this can increase the capacity factor of different energy resources. Increased capacity factor of efficient energy resources can reduce overall system cost by increasing the value of capital investments. 
Table 5. Ratio of Maximum to Minimum Loads

\begin{tabular}{|l|l|l|l|}
\hline Year & Minimum Demand (MW) & Maximum Demand (MW) & Ratio \\
\hline 2008 & 1,552 & 4,160 & 2.68 \\
\hline 2009 & 1,635 & 4,312 & 2.64 \\
\hline 2010 & 1,714 & 4,745 & 2.77 \\
\hline 2011 & 1,774 & 4,909 & 2.77 \\
\hline 2012 & 2,054 & 5,248 & 2.56 \\
\hline 2013 & 2,346 & 5,464 & 2.33 \\
\hline 2014 & 2,529 & 5,628 & 2.23 \\
\hline 2015 & 2,495 & 5,800 & 2.32 \\
\hline 2016 & 2,681 & 6,027 & 2.25 \\
\hline 2017 & 2,691 & 6,170 & 2.29 \\
\hline 2018 & 2,800 & 6,310 & 2.25 \\
\hline Texas 2018 & 27,139 & 73,308 & 2.70 \\
\hline
\end{tabular}

\subsection{NREL Short-Term Load Forecast for ONEE}

Short-term electricity load forecasting is important for planning day-to-day power generation scheduling, electricity pricing, and transaction planning. Further, economic dispatch of power plants and ensuring reliability of the system are guided by short-term load forecasts (Kyriakides and Polycarpou, n.d.). Short-term load forecasting horizons vary from a few minutes to several days; geographic extents can vary from the whole country to small regions. Short-term electricity demand is mainly influenced by weather conditions, daily and weekly cycles, holidays, and special events.

Numerous techniques have been used for the short-term demand forecasting (Suganthi and Samuel 2012). Mainly, these techniques can be categorized into classical statistical methods and machine learning methods ( $\mathrm{Vu}$ et al. 2017). The statistical methods are transparent and can interpret behavior of variables and their influence on the underlying model. Meanwhile, machine learning methods capture the nonlinear patterns of the demand and can handle additional complexity. Statistical methods for time series data analysis such as auto regressive models, ARIMA, and SARIMA are popular in short-term demand forecasting. Some common load forecasting software also selects historical demand curves matching the parameters for the following days in terms of temperature and day type. On the other hand, machine learning techniques such as support vector machine, neural networks, and neural network combining with other techniques such as wavelet analysis and fuzzy functions have been used for several studies. Less transparency of the machine learning techniques leads to problems in understanding relationships of variables, as well as increased complexity (Weron 2006).

The seasonal auto regressive, moving average with the external variables method is used for Morocco electricity load forecasting. The details of the SARIMAX model are discussed in Section 3.1. The Morocco SARIMAX model captures the past values of hourly electricity demand, temperature, and seasonal effects of the variables. 
Figure 23 shows three patterns of daily demands of the Morocco 7-day week. Days of the week can be binned by categories that often depend on country-specific cultural reasons ${ }^{4}$. Friday and Saturday have approximately equal demand profiles that are lower than the other four days of the working week. Meanwhile, Monday, Tuesday, Wednesday, and Thursday have similar daily demand profiles, and Sunday has a different profile than the rest of the days of the week. Furthermore, the electricity demand of special holidays has been removed. A single-season SARIMAX model is used for the daily demand prediction.

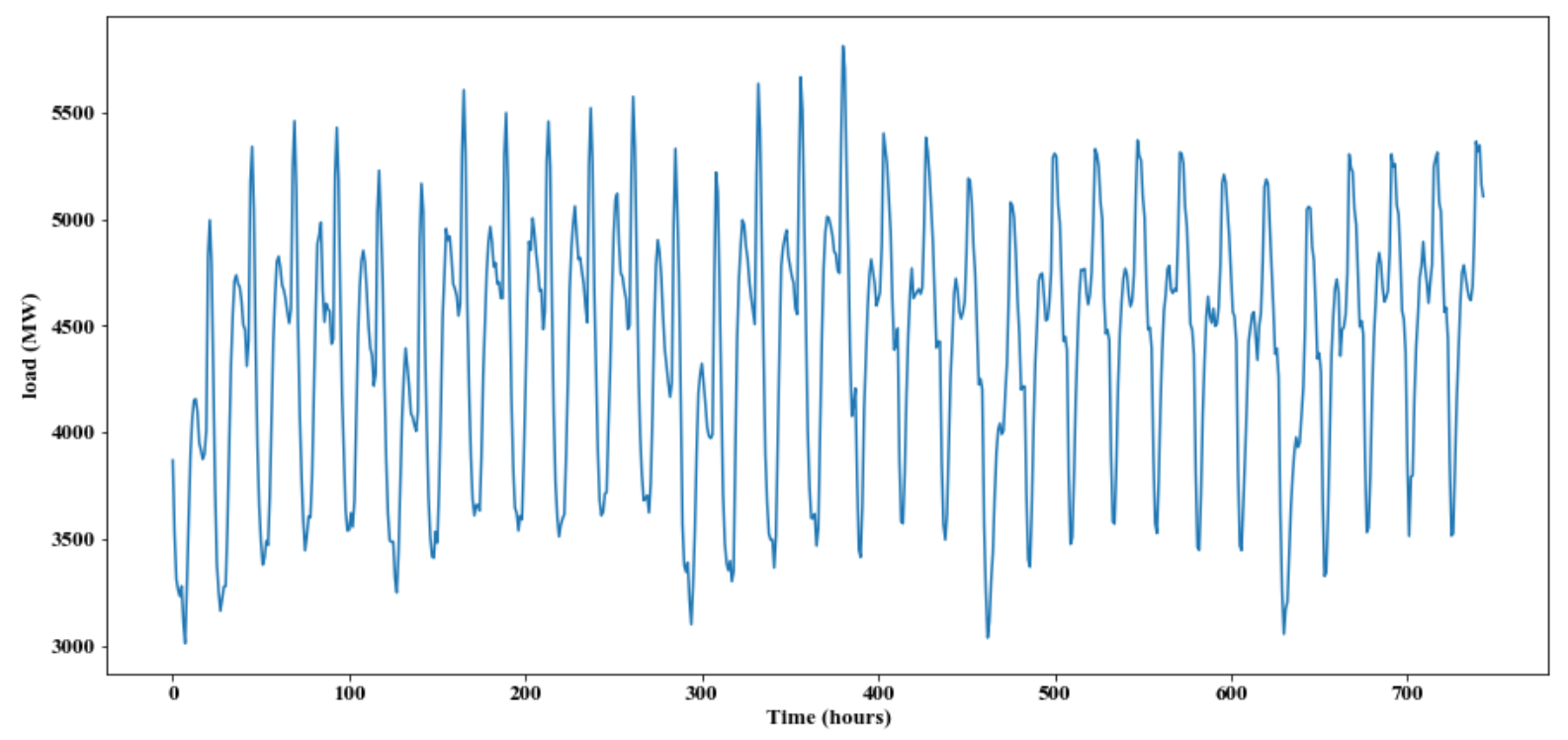

Figure 23. Hourly electricity demand of the Morocco Year 2018, December

Four years $(2015,2016,2017,2018)$ of hourly data are used for model training. Hourly electricity load and temperature data were divided into training $(85 \%)$ and testing $(15 \%)$ for time series data modeling. SARIMAX model training is carried out using all 4 years of data, as well as only using 2018 data. First, the autocorrelation function (ACF) and partial autocorrelation function (PACF) of the data series (Figure 24) were analyzed. ACF and PACF is the basis for determining $p$ and $q$ values of the time series model, where PACF gives significant auto regressive terms $p$ and ACF gives number of moving average terms $q$. High correlation of first few lags of electricity demand demonstrates daily seasonal characteristics. In addition, $168^{\text {th }}$ lag has slightly higher correlation than other higher order lags, which demonstrates weekly seasonal characteristics, as well.

\footnotetext{
${ }^{4}$ Morocco's dominant religion is Islam. Religious observances on Friday and Saturday affects the electricity demand. Sunday is not a working day but is also not considered unique as a religious day.
} 
(a) Autocorrelation of original series

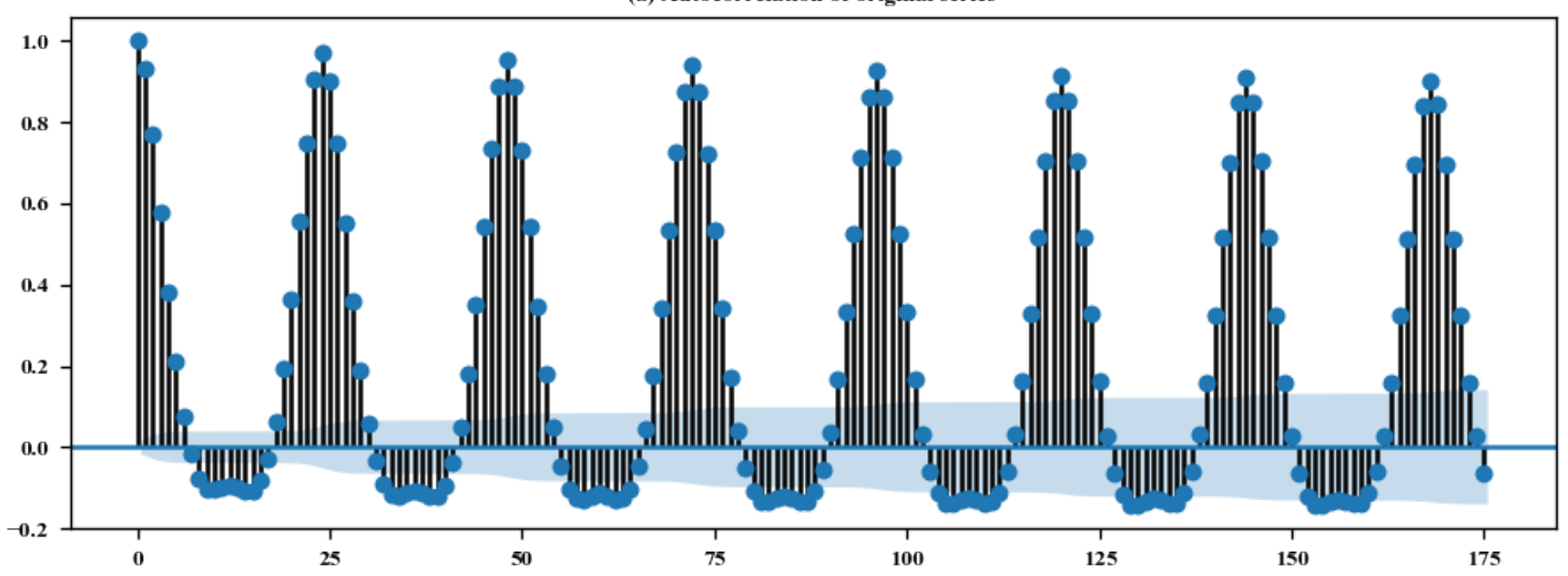

(b) Partial Autocorrelation of original series

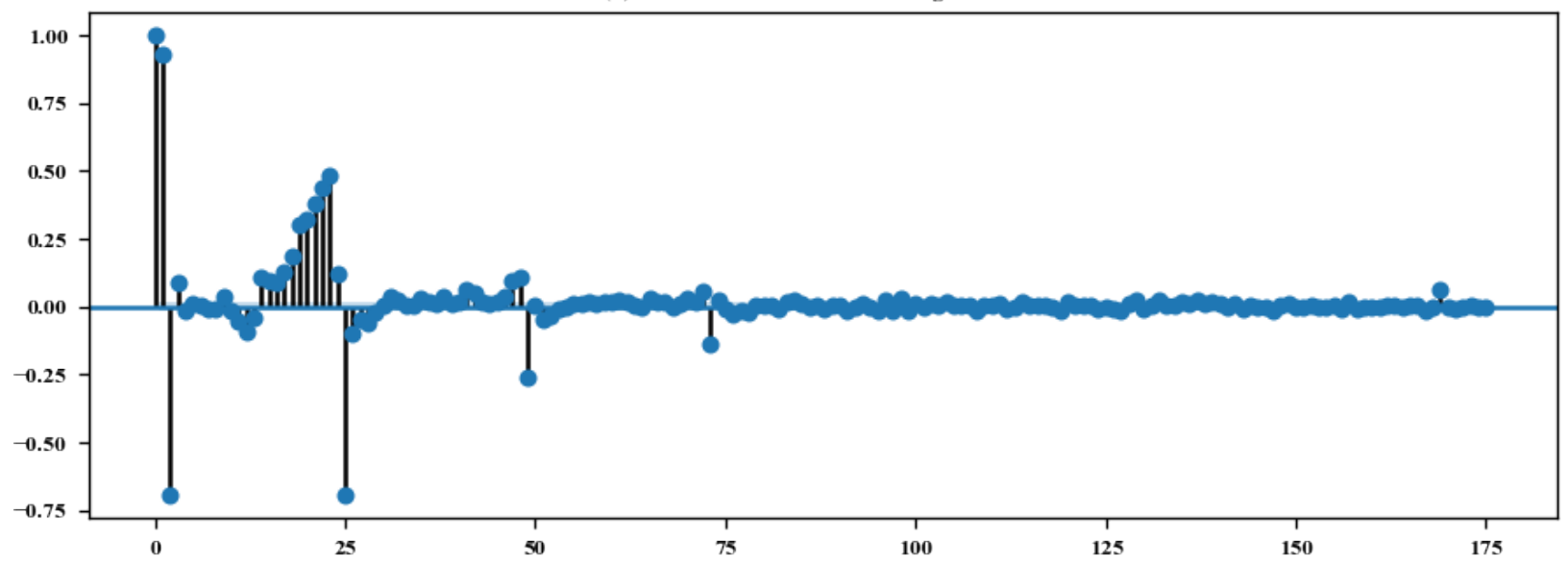

Figure 24. Hourly time series load data series for 2015-2018 demonstrates significant (a) autocorrelation and (b) partial correlation to the few lag hourly data electricity demand.

To select the parameters of SARIMAX model $(p, d, q, P, D, Q)$, Akaike information criterion (AIC) and Bayesian information criterion (BIC) are used. AIC and BIC guarantee the goodness of fit, while discouraging the overfitting of model with increasing number of parameters. AIC is calculated using maximum value of the log likelihood function and number of parameters of the model. BIC is calculated using number of data points, log likelihood function, and number of parameters. Better models have lower AIC and BIC values.

\subsubsection{Building of SARIMAX Models}

The SARIMAX is a powerful model for forecasting time series data; however, the tuning of model parameters is calculation-expensive. Hence, model parameters are fitted by the Auto.ARIMA tool while comparing the AIC and BIC values. The pyramid.arima package of python programming is used for building the forecasting models. The Auto.ARIMA function of the pyramid.arima package:

1. Builds the models for given parameter values

2. Calculates the AIC and BIC values

3. Selects the best fit model with the lowest AIC and BIC values (Table 6.). 
The same procedure for three categories of days is conducted, for 2015-2018 and only 2018 hourly electricity demand and temperature data. The same procedure is repeated without temperature data to compare the improvement forecasting accuracy. Altogether, 12 SARIMAX models were built for three categories of days. Training and testing errors of the four forecasting models for each category of day is compared.

Table 6. Building a SARIMAX Model for Monday, Tuesday, Wednesday, and Thursday Using 20152018 Hourly Load and Temperature Data

\begin{tabular}{|l|l|l|l|}
\hline$(p, d, q)$ & $(P, D, Q, S)$ & AIC & BIC \\
\hline$(1,0,1)$ & $(0,1,1,24)$ & 128351 & 128395 \\
\hline$(0,0,0)$ & $(0,1,0,24)$ & 144436 & 144458 \\
\hline$(1,0,0)$ & $(1,1,0,24)$ & 128691 & 128727 \\
\hline$(0,0,1)$ & $(0,1,1,24)$ & 135596 & 135632 \\
\hline$(1,0,1)$ & $(1,1,1,24)$ & 128350 & 128401 \\
\hline$(1,0,1)$ & $(1,1,0,24)$ & 128688 & 128732 \\
\hline$(1,0,1)$ & $(1,1,2,24)$ & 128343 & 128401 \\
\hline$(0,0,1)$ & $(1,1,2,24)$ & 135504 & 135555 \\
\hline$(2,0,1)$ & $(1,1,2,24)$ & 128342 & 128408 \\
\hline$(2,0,0)$ & $(1,1,2,24)$ & 128343 & 128401 \\
\hline$(2,0,2)$ & $(1,1,2,24)$ & 128200 & 128273 \\
\hline$(3,0,3)$ & $(1,1,2,24)$ & 128196 & 128284 \\
\hline$(3,0,3)$ & $(0,1,2,24)$ & 128202 & 128282 \\
\hline$(3,0,3)$ & $(2,1,2,24)$ & 128188 & 128283 \\
\hline$(3,0,3)$ & $(2,1,1,24)$ & 128183 & 128271 \\
\hline$(3,0,3)$ & $(1,1,0,24)$ & 128571 & 128644 \\
\hline$(2,0,3)$ & $(2,1,1,24)$ & 128180 & 128260 \\
\hline$(2,0,2)$ & $(2,1,1,24)$ & 128187 & 128260 \\
\hline$(1,0,2)$ & $(2,1,1,24)$ & 128253 & 128319 \\
\hline$(2,0,3)$ & $(1,1,1,24)$ & 128200 & 128273 \\
\hline$(2,0,3)$ & $(2,1,0,24)$ & 128192 & 128265 \\
\hline$(2,0,3)$ & $(2,1,2,24)$ & 128182 & 128269 \\
\hline$(2,0,3)$ & $(1,1,0,24)$ & 128567 & 128633 \\
\hline$(1,0,3)$ & $(2,1,1,24)$ & 128185 & 128258 \\
\hline & & & \\
\hline & & \\
\hline
\end{tabular}

\subsubsection{Comparison of Model Result}

The forecasting results of four SARIMAX models, which were built using the different data sets, are not unique and overlapped in their load forecasting estimation. The best fit SARIMAX models from different years of load and temperature data combination are also different from each other (Table 7). Results indicated Monday-Thursday forecast from the model built from longer period (Figure 25 (a) and (b)) are closer to the actual comparison to the forecasts from the models of 
2018-only data (Figure 25 (c) and (d)). On the other hand, Sunday's 24-hour forecast from the 2018 load data model is closer to the actual than others (Figure 25 (d)). However, it is hard to conclude which model is better than others because overall error of various SARIMAX models is in the same range (Table 8). Nevertheless, error values show some pattern, according to the data selection. Error percentage is calculated as the difference of actual load and forecasted load divided by the actual load. More than negative $50 \%$ of error values indicated that the models of 2015-2018 data forecast are generally higher than the actual load. On the other hand, models from 2018 data forecast less than actual, and more than $50 \%$ error values are positive.

Importance of regional-level sensitivity of temperature to the load is highlighted from the result. Substantial improvement of the result from the models with average temperature data cannot be noticed. Electricity loads vary with temperature in both positive and negative ways over the seasons. For example, summer air conditioning load is increased for higher temperatures, and winter heating load is increased for lower temperatures. Consideration of spatial and temporal temperature variation and sensitivity to the load might improve the short-term load forecast.

Table 7. Time Series Models Built Using Temperature and Load Data of Different Years Combination

\begin{tabular}{|l|l|l|l|}
\hline Data for Training the Model & MTWR & FS & Sunday \\
\hline 2015-2018 T\&L* & $(2,0,3)(2,1,1,24)$ & $(4,0,2)(1,1,2,24)$ & $(1,0,1)(2,1,2,24)$ \\
\hline 2015-2018 $\mathrm{L}^{* *}$ & $(1,0,0)(2,1,1,24)$ & $(2,0,3)(2,1,1,24)$ & $(1,0,1)(2,1,2,24)$ \\
\hline 2018 T\&L* $^{*}$ & $(4,0,2)(0,1,1,24)$ & $(1,0,3)(1,1,1,24)$ & $(2,0,3)(0,1,2,24)$ \\
\hline $2018 \mathrm{~L}^{* *}$ & $(3,0,2)(0,1,1,24)$ & $(2,0,2)(1,1,2,24)$ & $(1,0,1)(2,1,2,4)$ \\
\hline
\end{tabular}

T\&L*: temperature and load data are used, $\mathrm{L}^{* *}$ : load data is used

Table 8. Error Percentage Between Actual Load and Forecasted Load From Different Models

\begin{tabular}{|l|l|l|l|l|}
\hline Data & (a) 2015-2018 T\&L & (b) 2015-2018 L & (c) 2018 T\&L & (d) $2018 \mathrm{~L}$ \\
\hline Mean & -2.42 & -2.69 & 2.09 & 1.28 \\
\hline Std & 5.13 & 5.28 & 3.72 & 3.82 \\
\hline Minimum & -25.82 & -23.71 & -7.64 & -8.16 \\
\hline $25 \%$ & -4.00 & -4.69 & -0.52 & -1.3 \\
\hline $50 \%$ & -1.62 & -1.63 & 2.88 & 1.9 \\
\hline $75 \%$ & 0.44 & 0.42 & 4.54 & 4.18 \\
\hline Maximum & 6.26 & 7.25 & 12.77 & 10.05 \\
\hline
\end{tabular}



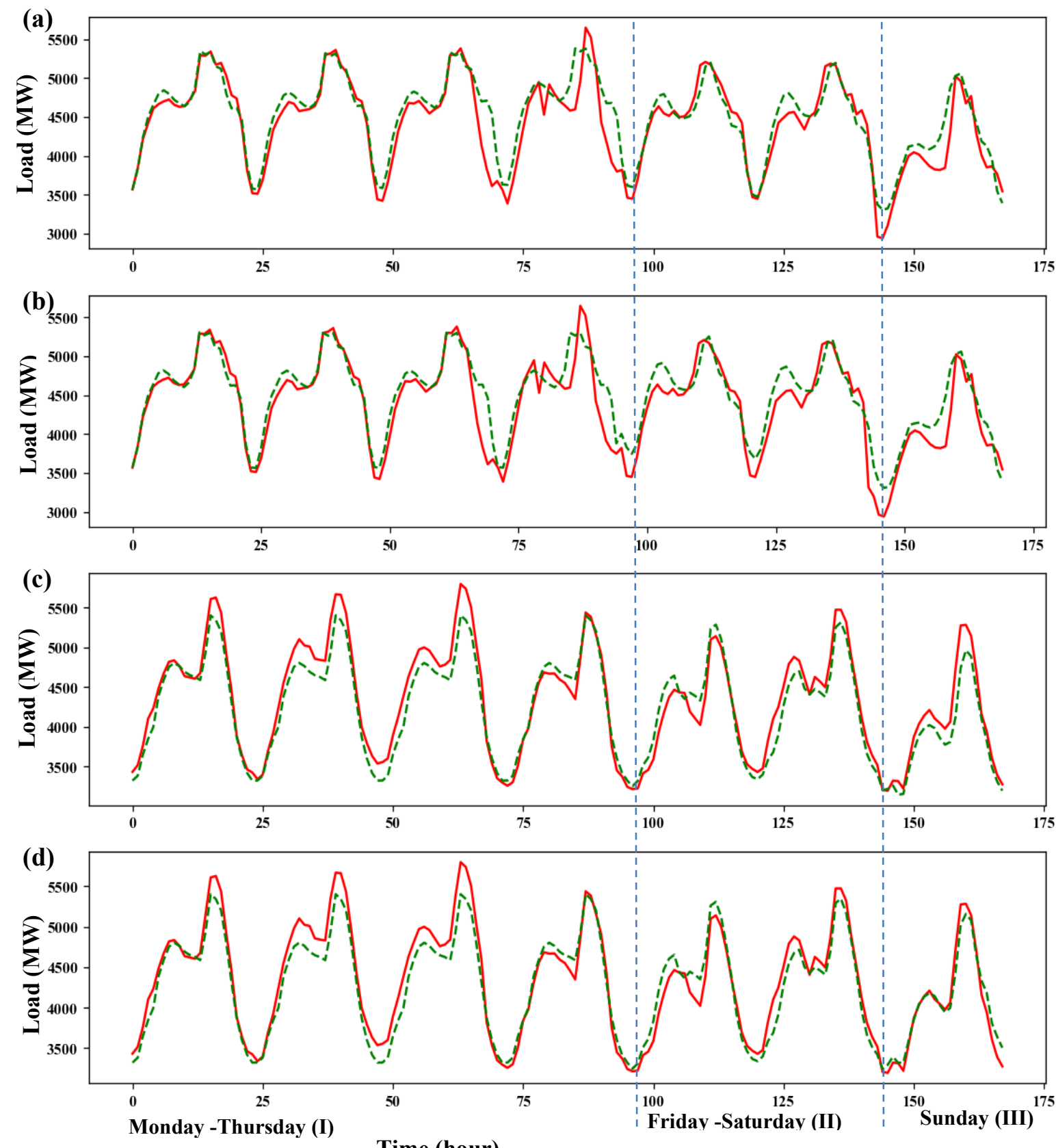

Time (hour)

Figure 25. Forecasting of seven days ahead using three models of SARIMA for Monday to Thursday (MTWR), Friday and Saturday (FS), and Sunday, built with (a) 2015-2018 hourly temperature and load, (b) 2015-2018 hourly load data, (c) 2018 hourly temperature and load, and (d) 2018 hourly load 
(a)

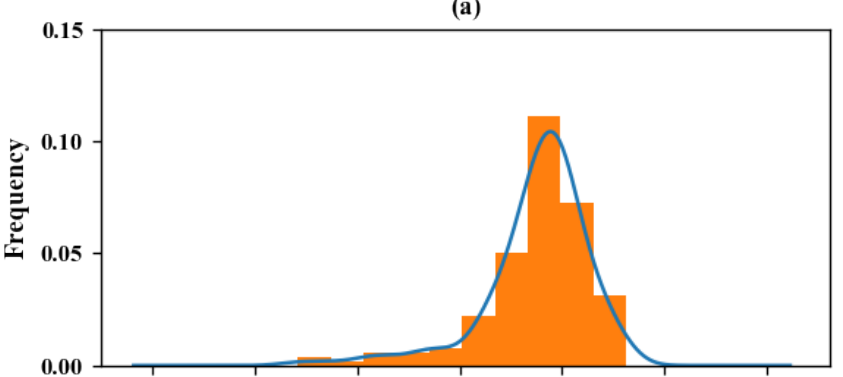

(c)

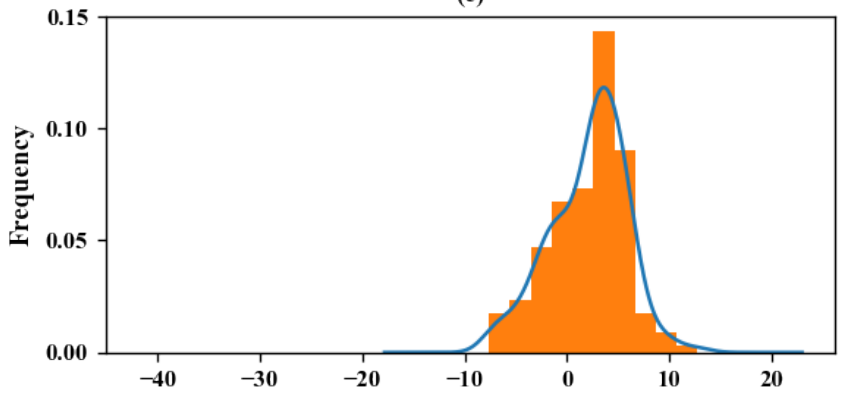

(b)

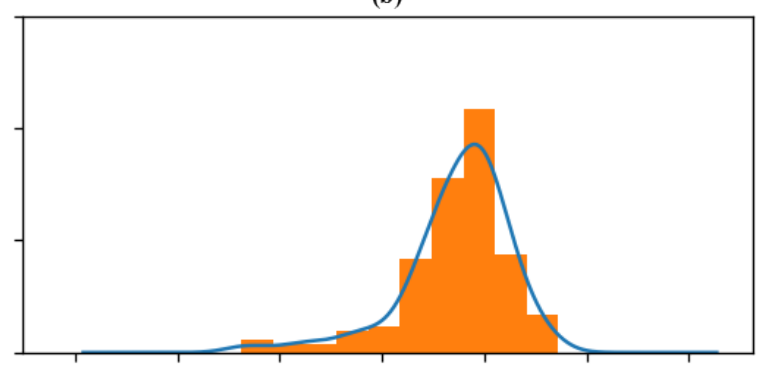

(d)

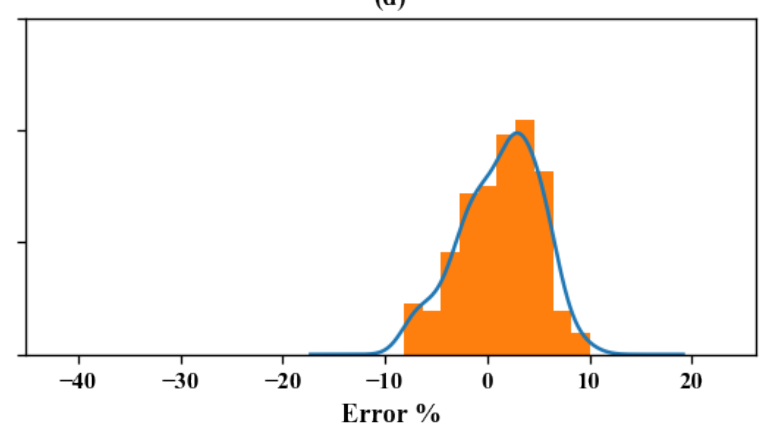

Figure 26 Forecasting error distribution for three models (MTWR, FS, Sunday) built using (a) 2015-2018 hourly temperature and load, (b) 2015-2018 hourly load, (c) 2018 hourly temperature and load data, and (d) 2018 hourly load 


\section{Additional Considerations for ONEE Load Forecasting}

Morocco's goals for the future include reducing dependence on foreign energy imports and reducing emissions from the electricity sector while enabling technologies such as air conditioning and electric vehicles to become widespread ("Morocco Gets Closer to 2020 Renewable Energy Objective" n.d.). In this section, several considerations are examined for factors influencing load forecasting beyond the techniques discussed in the previous section that may help guide Morocco to meeting these goals. The discussion includes utilizing additional data to establish relationships, utilizing advanced mathematical methods, and incorporating how certain technologies, such as energy efficiency and electrified vehicles may impact future loads. These considerations are potential extensions of this work.

\subsection{Data Requirements}

Incorporating known variables that affect electricity demand is a well-established technique for increasing the accuracy of load forecasting. Several examples of known variables include weather data, population, and known load anomalies such as holidays and weekends.

Weather is particularly important for load forecasting because some of the largest aggregated loads come from weather-dependent appliances such as air conditioning (Steinberg et al. 2020). Additionally, weather events can also affect thermal power plant operations. On the supply side, traditional thermal generators such as coal, natural gas, and nuclear power plants often reject heat to ambient heat sinks. These sinks can include bodies of water or the air. As the ambient temperature rises, these power plants' thermal efficiency decreases, meaning at high temperatures thermal plants can have reduced output (Sa 2011; Miara et al. 2017). Weather data should not only include temperature data but also solar radiation and wind speed and direction. In the medium and short terms, this data can be incorporated into decisions for the next few months and the day-ahead market (El Mghouchi, Ajzoul, and El Bouardi 2016; Fay and Ringwood 2010; Taylor and Buizza 2002; Sharma et al. 2014). For long-term planning, incorporating all weather-related impacts may impact investment decisions. When highresolution weather data is not available locally, there are multiple smoothing functions and artificial intelligence methods to use available data to produce the best possible load forecast (Singh and Khatoon 2012).

Understanding holidays, weekends, and major events that significantly affect electricity demand can also help increase the accuracy of load forecasts (Song et al. 2005). For instance, weekends and some holidays do not fall on the same date each year. A large chunk of error in the normalized load models provided in this report are likely because all days were regressed with no knowledge of these inconsistencies, except in the case of short-term modeling.

\subsection{Advanced Mathematical Algorithms}

The majority of this report employed relatively simplistic algorithms for either single or multivariable regression. Additionally, when AI and neural network algorithms were applied, they lacked some of the large data sets to produce a truly robust load forecast that could span across all timescales. Some of the advanced algorithms that could be pursued by research 
institutions in the future for more robust and advanced load forecasting are summarized in this section.

Load forecasting techniques that have been applied for years in the utility space include both a smoothing and iterative techniques. Smoothing techniques focus on taking historical data, taking specific timesteps (such as seasonal), developing a standardized "smooth" curve, and then updating the curve as the time period shrinks or more data becomes available (Broadwater et al. 1997; Infield and Hill 1998; El-Keib, Ma, and Ma 1995). A similar approach includes iteration on data and finding additional ways to reduce error. These algorithms often include some sort of automating and weighting of forecasts to reduce error. As part of bottom-up load forecasting, individual loads with the largest error and their relationship to, for example, weather events can be weighted to produce a more accurate forecast (Singh and Khatoon 2012).

Building on these traditional techniques are modifications that incorporate both novel concerns of the power system and the increased computing power that has been developed since the creation of centralized power systems. Several techniques can include auto regressive, stochastic, and adaptive. These modified methods include algorithms to produce load forecasts on the short term. Using measurements and automated systems, these algorithms can incorporate the most upto-date data, assess current errors, and adjust regression parameters dynamically (Gross and Galiana 1987; Lu et al. 1989; Grady et al. 1991; Paarmann and Najar 1995; Huang 1997; Barakat, Al-Qassim, and Rashed 1992; Chen, Wang, and Huang 1995).

The final category of load forecasting methodologies that will be mentioned in this report are artificial intelligence, machine learning, neural network algorithms, and genetic algorithms. In load forecasting today, machine learning algorithms are hailed as a major advancement. However, it is very important to understand where these methods do and do not work. The unifying aspect of this category of algorithms is that they can take in large amounts of data and correlate a system's behavior, especially when there is not a clear connection between physics and the system. Genetic algorithms are a good example of this. In genetic algorithms, an initial approach is identified, and a measure of success is defined (such as minimizing the error). The algorithm then attempts to correlate all the data and then is allowed to mutate in some small way but in an automated fashion. Many algorithms are tested, and the most successful is allowed to propagate and mutate (Singh and Khatoon 2012; Lee, Lee, and Chang 1997; Ma et al. 1995). In the case of all these methods, the actual correlation between the large data sets and the system behavior is a partial black box, and how the computer arrives at an optimum method is unknown to the programmer. Such methods are very well suited to the large data sets of today, but also can produce erroneous results that cannot be well interrogated, especially when the data set is too small. Therefore, such methods should be used with caution and by modelers with a thorough understanding of developing such algorithms. 


\section{Load Forecasting Methodologies for ONEE}

Aside from the load forecasting methodologies described above, there were several special considerations for the Moroccan electricity sector based on the country's state long-term energy goals. Below are described special considerations in load forecasting for incorporating country policies and goals for energy efficiency, transportation electrification, and per capita energy intensity changes over time.

\subsection{Impacts of Energy Efficiency in Long-Term Load Forecasting}

Utilities are treating energy efficiency like traditional power procurement in many of their longterm planning models. Importantly, target energy efficiency and demand side resources can be used to balance the system at key times and at key geographies, particularly if forecasting predicts specific load assets such as transformers will be overloaded for several hours a day during one season. Energy efficiency and demand-side resources can be used to shape the load so the system peak can be minimized without changing overall consumption or can be targeted to individual sectors that are stressing infrastructure. Energy efficiency measures can be offered by the utility, implemented as appliance or building standards, or incentivized through government rebate programs to help offset the initial costs (Sanstad 2014). In terms of load forecasting, energy efficiencies are often treated exogenously, or ex poste based on econometric load forecasts. In Section 9.3.2, load clustering was applied by arranging from highest to lowest, which gives insight into the ratio of peak to average demand. This can provide insight into potential methods to balance the system to reduce this ratio, which can require costly, rarely used capacity.

Several large-scale studies of the potential value of energy efficiency have been conducted to estimate the capacity and value of employing energy efficiency measures. A detailed analysis of the value of energy efficiency includes the potential energy efficiency that could be gained based on locally employed technologies, such as air conditioning, the policies that would enable a widespread adoption of those technologies (e.g., building codes, appliance codes, and awards programs such as energy start certification are examples of government enabled adoption of energy efficiency technologies), and finally a cost to implement that results in a $\$ / \mathrm{kW}$ and $\$ / \mathrm{kWh}$ estimate of the value of energy efficiency. In this way, energy efficiency can be treated and compared to traditional generators, and often is evaluated to be one of the most cost-efficient options for balancing system supply and demand (Hostick 2012; Hostick et al. 2014).

Historical Morocco data can be used from Table 9 to provide an estimate the impact of energy efficiency programs. Several studies examined the impact of specific appliances on the overall demand curve in different regions of the United States (Li and Just 2018; Sanstad 2014; Gumerman and Vegh 2019). The energy efficiency programs modeled produced anywhere between $1.7 \%$ to $25 \%$ energy reduction of household loads. ${ }^{5}$ Similar reductions are applied in Morocco to gauge the impact - specifically, examining the potential impact of $5 \%, 10 \%$, and $20 \%$ annual overall household demand reductions starting in 2010. Table 9 summarizes the impact of these reductions on overall annual energy consumption and uses a reference price of $\$ 0.02 \mathrm{USD} / \mathrm{kWh}$ to estimate the value of energy efficiency, which is within the range of

\footnotetext{
${ }^{5}$ There were some additional studies that examined upwards of $40 \%$ energy efficiency (Sanstad 2014), but these

"aggressive" outliers are excluded here.
} 
estimates (Wilson et al. 2017). This cost can be compared to the avoided cost of additional energy generation capacity to establish a net present value of such measures.

Table 9. Effect of Household Energy Efficiency Measures on Overall Annual Energy Consumption

\begin{tabular}{|l|l|l|}
\hline $\begin{array}{l}\text { Overall \% Annual Household Energy Efficiency } \\
\text { Reductions Beginning in } 2010\end{array}$ & $\begin{array}{l}2019 \% \text { Overall Annual } \\
\text { Reduction }\end{array}$ & $\begin{array}{l}\text { Energy System Cost } \\
\text { Reduction at \$0.02/kWh }\end{array}$ \\
\hline $5 \%$ & $3.51 \%$ & $\sim \$ 5.4$ million USD \\
\hline $10 \%$ & $7.01 \%$ & $\sim \$ 10.7$ million USD \\
\hline $20 \%$ & $14.03 \%$ & $\sim \$ 21.4$ million USD \\
\hline
\end{tabular}

\subsection{Electrifying Transportation}

Electrifying transportation can have many positive impacts. Electric transportation can reduce air pollution, interact positively with the electric grid, reduce consumer's overall transport costs, and reduce dependence on fossil fuels for transportation. Electric vehicles come in many forms, such as taxis, scooters, electric bikes, rented vehicles, personally owned vehicles, or electrified public transport such as buses and trains. Each of these technologies comes with its own set of tradeoffs and impacts that should be carefully considered in both electric, urban, and regulatory planning.

Several studies were conducted in the United States to look at the emissions reductions achieved by electric vehicle adoption with no change to customer behavior, as well as forecasts that look at hourly demand, assuming some level of utility control or pricing over charging (Kapustin 2020; Fitzgerald, Nelder, and Newcomb 2016; Wu et al. 2015).

In large economies with developing urban regions, 2-3 wheeled vehicles can account for between 5\%-20\% of fuel demand (Kapustin 2020). In contrast, in the United States, the personal vehicle fleet is approximately $60 \%$ light-duty vehicles that are often the focus of personal vehicle electrification (Hostick 2012). One approach to correct for this is to use the vehicle miles travelled of a country and multiply the projected percentage adoption of electric vehicles by the miles per kWh efficiency and electric vehicle adoption.

\section{Electricity Demand $\approx \%$ EV adoption $\times$ EV Efficiency $\times$ Annual VMT}

This approach could potentially exclude several key factors. For instance, it assumes that behaviors stay constant as electric vehicles are adopted. Additionally, there is a large range of efficiencies based on routes travelled, highway vs. urban driving, etc. One study placed the energy efficiency of electric vehicles in terms of their miles $/ \mathrm{kWh}$ between $2.8-5.2 \mathrm{miles} / \mathrm{kWh}$ based on daily routes and weather, with an average estimate for interstate driving at 3.7 miles/kWh (Wu et al. 2015). This difference can be significant under millions of vehicle miles travelled. An additional complication is that many customers prefer plug-in hybrid electric vehicles where there is both a battery and internal combustion engine to alleviate some of the range anxiety associated with electric vehicles over a 100\% battery-powered vehicle (Kurani, Sperling, and Turrentine 1996), which implies only some fraction of vehicle miles travelled rely on electricity. Finally, the charging location of the electric vehicle affects electricity demand 
significantly. If electric vehicles are encouraged in rural vs. urban environments, the daily miles driven can be significantly different than the percentage of electric vehicle adoption.

A detailed demand study for United States estimated an electric vehicle saturation at $40 \%$ of light-duty vehicles by 2050, with annual energy consumed by electric vehicles of $350 \mathrm{TWh}$ (Hostick 2012). Applying similar studies to Morocco will require a deep understanding of customer behavior, urbanization, current transportation usage, electric adoption, and policies to encourage both electric vehicle adoption and off-peak charging behaviors. Additional consideration needs to be considered for publicly available infrastructure to support electric vehicles. Electric vehicles can be charged at home, over several hours at level 2 public parking such as shopping centers and workplaces, and over and hour at a level 3 fast charger. The policy of charging infrastructure deployment and domestically available electric vehicle models all affect transportation electrification (Jadun et al. 2017; Hostick et al. 2014; P Denholm and Short 2006; Sears, Glitman, and Roberts 2014).

\subsection{Impacts of Other Technology Deployment in Long-Term Load Forecasting}

Individual energy intensity is the per capita energy consumption, and it can also be used to estimate a per household energy consumption. ${ }^{6}$ As countries increase their wealth, citizens often increase their energy intensity through lifestyle choices, such as the adoption of air conditioning. This trend has been observed in other countries and can benefit from existing data and by identifying where on the energy intensity curve the average and median Moroccan lies.

As an example of this, in 2018, the annual consumption of electricity in the United States was 10,970 kWh/household compared to $992 \mathrm{kWh} /$ household in Morocco ("Frequently Asked Questions (FAQs) - U.S. Energy Information Administration (EIA)" 2019; "Form EIA-860 Detailed Data with Previous Form Data (EIA-860A/860B)" 2019). A significant portion of household electricity consumption in the United States comes from air conditioning load. For instance, in the West South Central region of the United States, which has a similar climate to Morocco, the average annual cooling consumption is $4,000 \mathrm{kWh} /$ household ("Residential Energy Consumption Survey (RECS) - Energy Information Administration" 2015; "Form EIA-860 Detailed Data with Previous Form Data (EIA-860A/860B)" 2019). The per capita energy demand in Morocco is substantially lower than in the States. However, the United States has much larger penetration of air conditioning appliances which clearly increases the per capita demand. An increased deployment of air conditioning (or similar technologies) in Morocco may lead to an increase in the intensity.

\footnotetext{
${ }^{6}$ Note that, strictly speaking, energy intensity refers to total energy or primary energy. However, in this context, it refers to electricity energy intensity.
} 


\section{Conclusions and Recommendations for Future Work}

This report provides an overview of load forecasting methods and initial analysis for load forecasting within the Moroccan electricity sector, along with several recommendations for next steps. The techniques and considerations in this report detail practices that may be useful for future efforts in load forecasting in Morocco, in addition to efforts already underway at ONEE.

Firstly, identified below are considerations for three temporal facets of load forecasting:

- Long-term load forecasting (here, through 2030). ONEE's own analysis as well as GDP-coupled projections predict significant increases in the annual energy consumption in Morocco through 2030. GDP growth is generally accompanied by a growth in consumption, although some countries have decoupled this relationship to some degree through energy efficiency measures and alternative technologies that decrease energy intensity. However, the deployment of certain technologies (such as air conditioning, electric vehicles, and customer-owned solar) may impact long-term load, as well as daily load shapes. The factors that influence the diffusion rate of these technologies into society is complicated and dependent on consumer behavior, technology development, and even subsidies and rate design. So, a bottom-up analysis of load forecasts, incorporating some of these complicated factors, may provide additional insight.

- Medium-term load forecasting (a year into the future). The analysis of medium-term forecasting methods shows useful several regression methods and the associated error. These forecasting methods attempt to capture some of the season variation that can occur at each hour of the year, and the individual growth rates of each hour. These regression methods may be useful for medium-term load forecasting in Morocco. Additionally, by clustering the load days by their peak demand, a measure of the flatness of the energy system when comparing peak to average and peak to minimum load can be obtained. An electricity system with a high peak to average and peak to minimum ratio can suggest an inefficient electricity system where some capacity is only called on a handful or few dozen hours a year. This capacity is the costliest and can likely be displaced or better utilized by flattening the energy demand. Policy decisions such as energy efficiency measures and time-of-use rates can be low-cost pathways to flattening, among other mechanisms.

- Short-term load forecasting (minutes, hours, or days into the future). Several machine learning, auto regression, and neural network applications were used to predict electricity load over the next 24 hours and tested based on historical data. In particular, weather patterns, Morocco's current and projected future population, city load centers, and bottom-up load decomposition data was identified as important data sources which may increase robustness of the short-term load forecasting. There is significant opportunity for Moroccan energy authorities to become a leader in this space due to country size and high dependence on weather of both electricity demand and production. Increasing the data collection and availability as described in each load forecasting section would allow more entities to examine and experiment on different load forecasting algorithms. While several algorithms were applied to construct short-term 
load forecasting models, others such as genetic algorithms and support vector techniques that have been successfully applied by other authors should be investigated. Although often sufficient for short-term load forecasting, as bottom-up data becomes more available, these data-intensive techniques can be expanded.

In addition to the discussions on short-, medium-, and long-term load forecasting, several options for potential work were identified. These include collecting additional data (such as highresolution weather data) and more complex computational methods (such as those employing artificial intelligence or neural network algorithms). Given that Morocco has significant ambitions for its renewable energy deployment, valuable areas for future work include the expansion of tools such as the Renewable Energy Data Explorer. Also detailed were several considerations which could dramatically impact future loads, including energy efficiency and electric vehicles. There exists ongoing work in the field of technology diffusion, which may help frame possible future scenarios for Morocco's power system.

As Morocco undergoes many changes in their energy system over the coming decades, it will be important to consider both technologies and policies that support a reliable, affordable, and sustainable electricity sector. 


\section{References}

Abu-Shikhah, Nazih, Fawwaz Elkarmi, and Osama M. Aloquili. 2011. "Medium-Term Electric Load Forecasting Using Multivariable Linear and Non-Linear Regression." Smart Grid and Renewable Energy 02 (02): 126-35. https://doi.org/10.4236/sgre.2011.22015.

Arora, Siddharth, and James W. Taylor. 2016. "Forecasting Electricity Smart Meter Data Using Conditional Kernel Density Estimation." Omega, Business Analytics, 59 (March): 47-59. https://doi.org/10.1016/j.omega.2014.08.008.

Barakat, E. H., J. M. Al-Qassim, and S. A. Al Rashed. 1992. "New Model for Peak Demand Forecasting Applied to Highly Complex Load Characteristics of a Fast Developing Area." IEE Proceedings C (Generation, Transmission and Distribution) 139 (2): 136-40. https://doi.org/10.1049/ipc.1992.0022.

Bary, Constantine. 1945. "Coincidence-Factor Relationships of Electric-Service-Load Characteristics." Electrical Engineering 64 (9): 623-28. https://doi.org/10.1109/EE.1945.6441255.

Bennett, Christopher, Rodney Stewart, and Junwei Lu. 2014. “Autoregressive with Exogenous Variables and Neural Network Short-Term Load Forecast Models for Residential Low Voltage Distribution Networks." Energies 7 (5): 2938-60. https://doi.org/10.3390/en7052938.

Bentaibi, Wacef, Benoit Pape, Benjamin Jothy, and Gide Loyrette Nouel. n.d. "Electricity Regulation in Morocco: Overview Q\&A." Practical Law. Accessed March 25, 2020.

http://uk.practicallaw.thomsonreuters.com/w-0193058? originationContext=document $\&$ transitionType=Documentltem \&contextData=(sc.Default $)$ \&firstPage=true \&bhcp $=1$.

Bhattacharyya, Subhes C., and Govinda R. Timilsina. 2009. "Energy Demand Models for Policy Formulation : A Comparative Study of Energy Demand Models." Policy Research Working Paper WPS 4866. Washington, D.C.: World Bank. http://documents.worldbank.org/curated/en/800131468337793239/Energy-demand-modelsfor-policy-formulation-a-comparative-study-of-energy-demand-models.

Bozkurt, Ömer Özgür, Göksel Biricik, and Ziya Cihan Tayşi. 2017. "Artificial Neural Network and SARIMA Based Models for Power Load Forecasting in Turkish Electricity Market." Edited by Xiaosong Hu. PLOS ONE 12 (4): e0175915. https://doi.org/10.1371/journal.pone.0175915.

Broadwater, R.P., A. Sargent, A. Yarali, H.E. Shaalan, and J. Nazarko. 1997. "Estimating Substation Peaks from Load Research Data." IEEE Transactions on Power Delivery 12 (1): 451-56. https://doi.org/10.1109/61.568270.

Carvallo, Juan Pablo, Peter H. Larsen, Alan H. Sanstad, and Charles A. Goldman. 2016. "Load Forecasting in Electric Utility Integrated Resource Planning."

Chen, Jiann-Fuh, Wei-Ming Wang, and Chao-Ming Huang. 1995. "Analysis of an Adaptive Time-Series Autoregressive Moving-Average (ARMA) Model for Short-Term Load Forecasting." Electric Power Systems Research 34 (3): 187-96. https://doi.org/10.1016/0378-7796(95)00977-1.

"Chiffre Clés 2019 Energie Electrique." 2019. Office Nationale de L’Electricité et de L'Eau Potable.

Cortes, C, and V Vapnik. n.d. "Support-Vector Networks." Machine Learning 20 (3): 273-97.

Cox, Sadie, Anthony Lopez, Andrea Watson, Nick Grue, and Jennifer E. Leisch. 2018. "Renewable Energy Data, Analysis, and Decisions: A Guide for Practitioners." Technical Report NREL/TP-6A20-68913. Golden, CO: National Renewable Energy Laboratory (NREL). https://www.nrel.gov/docs/fy18osti/68913.pdf.

Denholm, P, and W Short. 2006. "Evaluation of Utility System Impacts and Benefits of Optimally Dispatched Plug-In Hybrid Electric Vehicles (Revised)." Hybrid Electric Vehicles, October, 30. 
Denholm, Paul, Matthew O'Connell, Gregory Brinkman, and Jennie Jorgenson. 2015. “Overgeneration from Solar Energy in California. A Field Guide to the Duck Chart." NREL/TP--6A20-65023, 1226167. https://doi.org/10.2172/1226167.

El Khchine, Younes, Mohammed Sriti, and Nacer Eddine El Kadri Elyamani. 2019. "Evaluation of Wind Energy Potential and Trends in Morocco." Heliyon 5 (6): e01830. https://doi.org/10.1016/j.heliyon.2019.e01830.

El Mghouchi, Y., T. Ajzoul, and A. El Bouardi. 2016. "Prediction of Daily Solar Radiation Intensity by Day of the Year in Twenty-Four Cities of Morocco." Renewable and Sustainable Energy Reviews 53 (January): 823-31. https://doi.org/10.1016/j.rser.2015.09.059.

El-Keib, A. A., X. Ma, and H. Ma. 1995. "Advancement of Statistical Based Modeling Techniques for Short-Term Load Forecasting." Electric Power Systems Research 35 (1): 51-58. https://doi.org/10.1016/0378-7796(95)00987-6.

Fay, Damien, and John V. Ringwood. 2010. "On the Influence of Weather Forecast Errors in Short-Term Load Forecasting Models." IEEE Transactions on Power Systems 25 (3): 1751-58. https://doi.org/10.1109/TPWRS.2009.2038704.

Feinberg, Eugene A., and Dora Genethliou. 2005. "Load Forecasting." In Applied Mathematics for Restructured Electric Power Systems: Optimization, Control, and Computational Intelligence, edited by Joe H. Chow, Felix F. Wu, and James Momoh, 269-85. Boston, MA: Springer US. https://doi.org/10.1007/0-387-23471-3_12.

Fitzgerald, Garrett, Chris Nelder, and James Newcomb. 2016. "ELECTRIC VEHICLES AS DISTRIBUTED ENERGY RESOURCES," June, 78.

"Form EIA-860 Detailed Data with Previous Form Data (EIA-860A/860B)." 2019. September 3, 2019. https://www.eia.gov/electricity/data/eia860/.

"Frequently Asked Questions (FAQs) - U.S. Energy Information Administration (EIA)." 2019. October 2, 2019. https://www.eia.gov/tools/faqs/faq.php.

Frew, Bethany, Gord Stephen, Devon Sigler, Jessica Lau, Wesley Jones, and Aaron Bloom. 2019. "Evaluating Resource Adequacy Impacts on Energy Market Prices across Wind and Solar Penetration Levels." The Electricity Journal 32.

"Global Solar Atlas." 2020. December 2020. https://globalsolaratlas.info/map.

Gneiting, Tilmann, and Matthias Katzfuss. 2014. "Probabilistic Forecasting." Annual Review of Statistics and Its Application 1 (1): 125-51. https://doi.org/10.1146/annurev-statistics-062713-085831.

Grady, W.M., L.A. Groce, T.M. Huebner, Q.C. Lu, and M.M. Crawford. 1991. "Enhancement, Implementation, and Performance of an Adaptive Short-Term Load Forecasting Algorithm." IEEE Transactions on Power Systems 6 (4): 1404-10. https://doi.org/10.1109/59.116982.

Gross, G., and F.D. Galiana. 1987. "Short-Term Load Forecasting." Proceedings of the IEEE 75 (12): 155873. https://doi.org/10.1109/PROC.1987.13927.

Gumerman, Etan, and Tibor Vegh. 2019. "Modeling Energy Efficiency as a Supply Resource: A Bottom-up Approach." Energy Efficiency, 13.

Hahn, Heiko, Silja Meyer-Nieberg, and Stefan Pickl. 2009. "Electric Load Forecasting Methods: Tools for Decision Making." European Journal of Operational Research, 6.

Hale, Elaine, Lori Bird, Rajaraman Padmanabhan, and Christina Volpi. 2016. "Potential Roles for Demand Response in High-Growth Electric Systems with Increasing Shares of Renewable Generation." NREL/TP-6A20-70630. Golden, CO: NREL. https://www.nrel.gov/docs/fy19osti/70630.pdf.

Herrick, James. 2016. "On Representation of Temporal Variability in Electricity Capacity Planning Models." Energy Economics 58: 261-74.

Hochberg, Michael. 2016. "Renewable Energy Growth in Morocco." https://www.mei.edu/sites/default/files/publications/PF26_Hochberg_Moroccorenewables_we b.pdf. 
Hong, Tao. 2014. "Energy Forecasting: Past, Present and Future." Foresight: The International Journal of Applied Forecasting, 43-48.

- - . 2016. "Probabilistic Electric Load Forecasting: A Tutorial Review." International Journal of Forecasting, 25.

Hong, Tao, Jason Wilson, and Jingrui Xie. 2014. "Long Term Probabilistic Load Forecasting and Normalization With Hourly Information." IEEE Transactions on Smart Grid 5 (1): 456-62. https://doi.org/10.1109/TSG.2013.2274373.

Hostick, D. 2012. "Renewable Electricity Futures Study. Volume 3: End-Use Electricity Demand," 132.

Hostick, D, D Belzer, S Hadley, T Markel, C Marnay, and M Kintner-Meyer. 2014. "Projecting Electricity Demand in 2050," July, 32.

Houimli, Rim, Mourad Zmami, and Ousama Ben-Salha. 2020. "Short-Term Electric Load Forecasting in Tunisia Using Artificial Neural Networks." Energy Systems 11: 357-75.

Huang, S. R. 1997. "Short-Term Load Forecasting Using Threshold Autoregressive Models." IEE Proceedings - Generation, Transmission and Distribution 144 (5): 477-81. https://doi.org/10.1049/ip-gtd:19971144.

Infield, D.G., and D.C. Hill. 1998. "Optimal Smoothing for Trend Removal in Short Term Electricity Demand Forecasting." IEEE Transactions on Power Systems 13 (3): 1115-20. https://doi.org/10.1109/59.709108.

IRENA. 2017. "Planning for the Renewable Future: Long-Term Modelling and Tools to Expand Variable Renewable Power in Emerging Economies." Abu Dhabi: International Renewable Energy Agency (IRENA). http://www.irena.org/publications/2017/Jan/Planning-for-the-renewable-future-Longterm-modelling-and-tools-to-expand-variable-renewable-power.

Jadun, Paige, Colin McMillan, Daniel Steinberg, Matteo Muratori, Laura Vimmerstedt, and Trieu Mai. 2017. "Electrification Futures Study: End-Use Electric Technology Cost and Performance Projections through 2050," 108.

Kapustin, Nikita O. 2020. "Long-Term Electric Vehicles Outlook and Their Potential Impact on Electric Grid." Energy Policy, 10.

Katz, Jessica, and Michael Milligan. 2016. "The Evolution of Power System Planning with High Levels of Variable Renewable Generation. Greening the Grid." Technical Report. Golden, CO: National Renewable Energy Laboratory (NREL). https://www.nrel.gov/docs/fy16osti/63035.pdf.

Khatib, Tamer, Azah Mohamed, and K. Sopian. 2013. "A Review of Photovoltaic Systems Size Optimization Techniques." Renewable and Sustainable Energy Reviews 22 (June): 454-65. https://doi.org/10.1016/j.rser.2013.02.023.

Kousksou, T., A. Allouhi, M. Belattar, A. Jamil, T. El Rhafiki, A. Arid, and Y. Zeraouli. 2015. "Renewable Energy Potential and National Policy Directions for Sustainable Development in Morocco." Renewable and Sustainable Energy Reviews 47 (July): 46-57. https://doi.org/10.1016/j.rser.2015.02.056.

Kurani, K., D. Sperling, and T. Turrentine. 1996. "The Marketability of Electric Vehicles: Battery Performance and Consumer Demand for Driving Range." In Proceedings of 11th Annual Battery Conference on Applications and Advances, 153-58. https://doi.org/10.1109/BCAA.1996.484986.

Kuster, Corentin, Yacine Rezgui, and Monjur Mourshed. 2017. "Electrical Load Forecasting Models: A Critical Systematic Review." Sustainable Cities and Society 35 (November): 257-70. https://doi.org/10.1016/j.scs.2017.08.009.

Kyriakides, Elias, and Marios Polycarpou. n.d. "Short Term Electric Load Forecasting: A Tutorial." In Trends In Neural Computation. Vol. 35.

Lee, Dong Gyu, Byong Whi Lee, and Soon Heung Chang. 1997. "Genetic Programming Model for LongTerm Forecasting of Electric Power Demand." Electric Power Systems Research 40 (1): 17-22. https://doi.org/10.1016/S0378-7796(96)01125-X. 
Li, Jia, and Richard E Just. 2018. "Modeling Household Energy Consumption and Adoption of Energy Efficient Technology." Energy Economics, 12.

Lu, Q.-C., W.M. Grady, M.M. Crawford, and G.M. Anderson. 1989. "An Adaptive Nonlinear Predictor with Orthogonal Escalator Structure for Short-Term Load Forecasting." IEEE Transactions on Power Systems 4 (1): 158-64. https://doi.org/10.1109/59.32473.

Ma, X., A. A. El-Keib, R. E. Smith, and H. Ma. 1995. "A Genetic Algorithm Based Approach to Thermal Unit Commitment of Electric Power Systems." Electric Power Systems Research 34 (1): 29-36. https://doi.org/10.1016/0378-7796(95)00954-G.

Miara, Ariel, Jordan Macknick, Charles Vorosmarty, Vincent Tidwell, Robin Newmark, and Balazs Fekete. 2017. "Climate and Water Resource Change Impacts Andadaptation Potential for US Power Supply." Nature Climate Change 7: 793-99.

Ministerio de Minas y Energía and Unidad de Planeación Minero Energética - UPME. 2018. "Plan de Expansion de Referencia Generacion- Transmision 2017 - 2031." http://www.upme.gov.co/Docs/Plan_Expansion/2017/Plan_GT_2017_2031.pdf.

"Modelling for Analysis of Energy Demand (MAED-2)." 2006. International Atomic Energy Agency.

Moral-Carcedo, Julián. 2017. "Integrating Long-Term Economic Scenarios into Peak Load Forecasting: An Application to Spain," 14.

"Morocco - Countries \& Regions." 2020. IEA. November 16, 2020. https://www.iea.org/countries/morocco.

"Morocco Gets Closer to 2020 Renewable Energy Objective." n.d. Accessed May 27, 2020. https://www.moroccoworldnews.com/2020/01/290604/morocco-closer-2020-renewableenergy-objective/.

“Morocco Renewable Energy Target 2030 - Policies." 2019. IEA. October 10, 2019. https://www.iea.org/policies/6557-morocco-renewable-energy-target-2030.

"Morocco Solar Program." n.d. LEDS Global Partnership. Accessed March 24, 2020. https://ledsgp.org/case-study/morocco-solar-program/.

Neukomm, Monica, Valerie Nubbe, and Robert Fares. 2019. "Grid-Interactive Efficient Buildings." DOE/ EE-1968. U.S. Dept. of Energy (USDOE), Washington DC (United States); Navigant Consulting, Inc., Chicago, IL (United States). https://doi.org/10.2172/1508212.

NGCP. 2018. "Philippine Transmission Development Plan: 2016-2040. Major Network Development Volume 1." Technical Report - Consultation Draft. Quezon City, Philippines: National Grid Corporation of the Philippines (NGCP). https://www.ngcp.ph/.

"Noor Ouarzazate Solar Complex, Morocco." n.d. Power Technology | Energy News and Market Analysis. Accessed June 22, 2020. https://www.power-technology.com/projects/noor-ouarzazate-solarcomplex/.

"ONEE Data, Site Web Officiel de l'ONEE - Branche Electricité." 2021. March 1, 2021. http://www.one.org.ma/.

Paarmann, Larry D., and Mohamed D. Najar. 1995. "Adaptive Online Load Forecasting via Time Series Modeling." Electric Power Systems Research 32 (3): 219-25. https://doi.org/10.1016/03787796(94)00918-T.

Palit, Debajit, and Akanksha Chaurey. 2011. "Off-Grid Rural Electrification Experiences from South Asia: Status and Best Practices." Energy for Sustainable Development, Special issue on off-grid electrification in developing countries, 15 (3): 266-76. https://doi.org/10.1016/j.esd.2011.07.004.

PDOE. 2017. "Philippine Power Development Plan 2016-2040." Taguig City, Philippines: Philippines Department of Energy (PODE). https://www.doe.gov.ph/electric-power/power-developmentplan-2016-2040. 
- - . 2018. "Philippines Energy Plan 2017-2040: Energy Demand \& Supply Outlook." Taguig City, Philippines: Philippines Department of Energy (PODE). https://www.doe.gov.ph/pep/philippineenergy-plan-2017-2040.

“PVWatts Calculator." 2021. March 29, 2021. https://pvwatts.nrel.gov/.

“Rapport D'Activités 2019 Energie Electrique." 2019. Office Nationale de L’Electricité et de L'Eau Potable.

"RE Explorer." 2021. January 2021. https://www.re-explorer.org/.

"Residential Energy Consumption Survey (RECS) - Energy Information Administration." 2015. 2015. https://www.eia.gov/consumption/residential/.

Sa, Ashley De. 2011. "Gas Turbine Performance at Varying Ambient Temperature." Applied Thermal Engineering, 5.

Sangrody, Hossein, and Ning Zhou. 2017. "An Initial Study on Load Forecasting Considering Economic Factors," March, 5.

Sanstad, Alan H. 2014. "Modeling an Aggressive Energy-Efficiency Scenario in Long-Range Load Forecasting for Electric Power Transmission Planning." Applied Energy, 12.

Scott, Ian J., Pedro M.S. Carvalho, Audun Botterud, and Carlos A. Silva. 2019. "Clustering Representative Days for Power Systems Generation Expansion Planning: Capturing the Effects of Variable Renewables and Energy Storage." Applied Energy 253 (November): 113603. https://doi.org/10.1016/j.apenergy.2019.113603.

Sears, Justine, Karen Glitman, and David Roberts. 2014. "Forecasting Demand of Public Electric Vehicle Charging Infrastructure." In 2014 IEEE Conference on Technologies for Sustainability (SusTech), 250-54. https://doi.org/10.1109/SusTech.2014.7046252.

Seiden, Ken, and Brian Eakin. 2017. "Solving The Utility Load Forecasting Conundrum: A New Framework." Public Utilities Fortnightly. https://www.navigant.com//media/www/site/insights/energy/2017/solving-the-utility-load-forecasting-conundrum-pu.pdf.

Seifi, Hossein, and Mohammad Sadegh Sepasian. 2011. "Load Forecasting." In Electric Power System Planning, by Hossein Seifi and Mohammad Sadegh Sepasian, 45-67. Berlin, Heidelberg: Springer Berlin Heidelberg. https://doi.org/10.1007/978-3-642-17989-1_4.

Senhaji, Faouzi. 2016. "Implementation of Morocco's Solar Energy Plan." Kigali, June 27. https://ledsgp.org/wp-content/uploads/2016/07/Implementation-of-Moroccos-solar-energyplan-Faouzi-Senhaji-.pdf.

Sharif, Islam, and Marufa Mithila. 2013. "Rural Electrification Using PV: The Success Story of Bangladesh." Energy Procedia, PV Asia Pacific Conference 2012, 33 (January): 343-54. https://doi.org/10.1016/j.egypro.2013.05.075.

Sharma, Navin, Jeremy Gummeson, David Irwin, Ting Zhu, and Prashant Shenoy. 2014. "Leveraging Weather Forecasts in Renewable Energy Systems." Sustainable Computing: Informatics and Systems 4 (3): 160-71. https://doi.org/10.1016/j.suscom.2014.07.005.

"SIE." 2021. April 6, 2021. http://www.lemonitor.com/en/projet/SIE/SIE_2.html.

Singh, Arunesh Kumar, and S Khatoon. 2012. "Load Forecasting Techniques and Methodologies: A Review," 10.

Song, K.-B., Y.-S. Baek, D.H. Hong, and G. Jang. 2005. "Short-Term Load Forecasting for the Holidays Using Fuzzy Linear Regression Method." IEEE Transactions on Power Systems 20 (1): 96-101. https://doi.org/10.1109/TPWRS.2004.835632.

Steinberg, Daniel, Bryan Mignone, Jordan Macknick, Yinong Sun, Kelly Eurek, Andrew Badger, Ben Livneh, and Kristen Averyt. 2020. "Decomposing Supply-Side and Demand-Side Impacts of Climate Change on the US Electricity System Through 2050." Climactic Change 158: 125-39. 
Steinbuks, Jevgenijs. 2017. "Assessing the Accuracy of Electricity Demand Forecasts in Developing Countries." WPS7974. The World Bank. http://documents.worldbank.org/curated/en/728681487169710866/Assessing-the-accuracy-ofelectricity-demand-forecasts-in-developing-countries.

Suganthi, L., and Samuel Samuel. 2012. "Energy Models for Demand Forecasting-A Review." Renewable and Sustainable Energy Reviews 16 (2): 1223-40. https://doi.org/doi.org/10.1016/j.rser.2011.08.014.

Taylor, J.W., and R. Buizza. 2002. "Neural Network Load Forecasting with Weather Ensemble Predictions." IEEE Transactions on Power Systems 17 (3): 626-32. https://doi.org/10.1109/TPWRS.2002.800906.

The Wind Power. 2017. "Tarfaya (Morocco)." 2017. https://www.thewindpower.net/windfarm_en_10765_tarfaya.php.

Turkay, Belgin, and Dilara Demren. 2011. "Electrical Load Forecasting Using Support Vector Machines." 2011 7th International Conference on Electrical and Electronics Engineering (ELECO).

Usman, Zainab, and Tayeb Amegroud. 2019. "Lessons from Power Sector Reforms: The Case of Morocco." The World Bank. https://doi.org/10.1596/1813-9450-8969.

Vu, D.H., K.M. Muttaqi, A.P. Agalgaonkar, and A. Bouzerdoum. 2017. "Short-Term Electricity Demand Forecasting Using Autoregressive Based Time Varying Model Incorporating Representative Data Adjustment." Applied Energy 205 (November): 790-801. https://doi.org/doi.org/10.1016/j.apenergy.2017.08.135.

Walton, Robert. 2018. "As Technology Upends Grid Fundamentals, Is Load Forecasting a Crapshoot?" Utility Dive. 2018. https://www.utilitydive.com/news/as-technology-upends-grid-fundamentalsis-load-forecasting-a-crapshoot/527969/.

Weron, Rafał. 2006. Modeling and Forecasting Electricity Loads and Prices: A Statistical Approach. Wiley Finance Series. Chichester, England; Hoboken, NJ: John Wiley \& Sons.

Wilson, Eric, Craig Christensen, Scott Horowitz, Joseph Robertson, and Jeff Maguire. 2017. "Energy Efficiency Potential in the U.S. Single-Family Housing Stock." Renewable Energy, 157.

Wu, Xinkai, David Freese, Alfredo Cabrera, and William A Kitch. 2015. "Electric Vehicles' Energy Consumption Measurement and Estimation," 16.

Xie, Jingrui, Tao Hong, and Joshua Stroud. 2015. "Long-Term Retail Energy Forecasting With Consideration of Residential Customer Attrition." IEEE Transactions on Smart Grid 6 (5): 224552. https://doi.org/10.1109/TSG.2014.2388078.

Yamaguchi, Kaoru. 2015. “[Simple] Economic Simulation System, for Excel: A Working Manual (Subject to Change Every Year) for Version 2015." Tokyo: The Institute of Energy Economics, Japan (IEEJ). http://prdrse4all.spc.int/system/files/simple_e_manual.pdf.

Zhang, Yao, Jianxue Wang, and Xifan Wang. 2014. "Review on Probabilistic Forecasting of Wind Power Generation." Renewable and Sustainable Energy Reviews 32 (April): 255-70. https://doi.org/10.1016/j.rser.2014.01.033. 